\title{
ÖSTERREICHISCHER TECHNOLOGIEBERICHT 1997
}

GERNOT HUTSCHENREITER, NORBERT KNOLL (WIFO), MANFRED PAIER, FRITZ OHLER (ÖFZS)

Studie des Österreichischen Instituts für Wirtschaftsforschung und des Österreichischen Forschungszentrums Seibersdorf

Eine Initiative des Bundesministeriums für wirtschaftliche Angelegenheiten und des Bundesministeriums für Wissenschaft und Verkehr

Mai 1998 


\title{
ÖSTERREICHISCHER TECHNOLOGIEBERICHT 1997
}

\author{
GERNOT HUTSCHENREITER, NORBERT KNOLL (WIFO), \\ MANFRED PAIER, FRITZ OHLER (ÖFZS)
}

\section{Inhaltsverzeichnis}

Einleitung

1. Das österreichische Forschungssystem im internationalen Vergleich

1.1 Niveau und Struktur der Aufwendungen für Forschung und Entwicklung 5

1.2 Finanzierung der Industrieforschung

1.3 Finanzierung von Forschung und Entwicklung im Hochschulsektor

1.4 Unternehmensfinanzierte Forschung und Entwicklung im öffentlichen Sektor

1.5 Die Vernetzung des Unternehmenssektors mit anderen Sektoren

2. Spezialisierungsmuster des österreichischen Innovationssystems

2.1 Humanressourcen

2.2 Patentspezialisierung

3. Internationale Technologieströme

3.1 Direktinvestitionen 36

3.2 Das technologische Profil des Außenhandels 37

3.3 Technologische Zahlungsbilanz 47

3.4 Internationale Produktivitätseffekte von Forschung und Entwicklung 50

4. Technologiediffusion im internationalen Vergleich 51

4.1 Informations- und Kommunikationstechnologie 52

4.2 Telekommunikationsinfrastruktur $\quad 58$

4.2.1 Das digitalisierte Telefonnetz als Basisinfrastruktur 58

4.2.2 Mobil- und Datenkommunikation 61

4.3 Informationstechnologie in der Produktion: Industrieroboter 62

4.4 Technologien für eine nachhaltige Entwicklung $\quad 70$

$\begin{array}{lll}\text { 4.4.1 Kombinierter Verkehr } & 71\end{array}$

4.4.2 Kraft-Wärme-Kopplung (Cogeneration) 74

5. Spezialteil: Österreich und die Rahmenprogramme der EU 80

6. Literatur 97 
- II - 


\section{Einleitung}

An den Österreichischen Technologiebericht 1997 war die Aufgabe gerichtet, einen Überblick über Fakten und wichtige Zusammenhänge im österreichischen Innovationssystem zu bieten. Dabei wird nicht nur auf nationale Informationsquellen zurückgegriffen, sondern auch auf eine Fülle internationaler Daten. Im Sinne von Benchmarking wird Österreichs Innovationssystem - wo immer dies möglich war - in eine internationale Perspektive gestellt. Der Österreichische Technologiebericht enthält keine technologiepolitischen Empfehlungen, sondern ist als Informationsquelle für die technologiepolitische Standortbestimmung und Diskussion gedacht. Er wendet sich damit sowohl an die Akteure des Innovationssystems aus Wirtschaft, Forschung, Politik und Verwaltung als auch an die an Fragen der Technologiepolitik interessierte Öffentlichkeit.

In einem generellen Sinn können das Niveau der Einkommen pro Kopf oder der Produktivität als die allgemeinsten Indikatoren für den technologischen Entwicklungsstand einer Wirtschaft angesehen werden. Hier hat Österreich beträchtliche Erfolge vorzuweisen. Österreich befindet sich heute, nach einem erfolgreichen wirtschaftlichen Aufholprozeß, hinsichtlich der Pro-Kopf-Einkommen und der Produktivität seiner Industrie im Spitzenfeld der Industrieländer. Als hochentwickeltes Industrie- und Hochlohnland sieht sich Österreich jedoch unter den Rahmenbedingungen einer sich globalisierenden Wirtschaft neuen Herausforderungen gegenüber. Österreich wird sich eine Position im Spitzenfeld nur sichern können, wenn es gelingt, sowohl durch den Einsatz neuer Technologien ein hohes Produktivitätsniveau zu erreichen als auch die Vorteile einer Spezialisierung auf humankapitalintensive und technologisch anspruchsvolle Produkte zu nutzen.

Die auf lange Sicht wichtigste Triebkraft des Wirtschaftswachstums ist nach den Erkenntnissen der Wirtschaftsforschung der technische Wandel. Private und öffentliche Investitionen in Forschung und Entwicklung (F\&E) spielen dabei eine zentrale Rolle. In der öffentlichen Diskussion steht zumeist die Höhe der Forschungsquote, d.h. der gesamtwirtschaftlichen Ausgaben für Forschung und Entwicklung in Relation zum Bruttoinlandsprodukt im Zentrum der Aufmerksamkeit. Tatsächlich gibt der Umstand, daß Österreichs Forschungsquote seit Anfang der neunziger Jahre auf dem international niedrigen Niveau von 1,5\% stagniert, Anlaß zur Diskussion. Ebenso wichtig ist jedoch die in der allgemeinen Diskussion zumeist vernachlässigte Struktur der Ausgaben für Forschung und Entwicklung. In Abschnitt 1 "Das österreichische Forschungssystem im internationalen Vergleich" werden daher wichtige strukturelle Zusammenhänge im österreichischen F\&ESystem dargestellt und einem internationalen Vergleich unterzogen. $\mathrm{Zu}$ den österreichischen Besonderheiten zählen insbesondere: der relativ geringe Anteil des Unternehmenssektors an der gesamten Forschung und Entwicklung, ein relativ großer Hochschulsektor, der einen weit über dem internationalen Durchschnitt liegenden Anteil der öffentlichen F\&E-Ausgaben absorbiert, sowie eine schwache Vernetzung der Komponenten des Innovationssystems. Die Internationalisierung der österreichischen Forschung, insbesondere des Hochschulsektors, ist nach wie vor relativ gering. Aus der Perspektive der 
Forschung über "Innovationssysteme", die diesem Bericht zugrundeliegt, sind die Intensität und Qualität des Zusammenwirkens zwischen den Elementen dieses Systems (Universitäten - Unternehmen - Forschungseinrichtungen) ein zentraler Ansatzpunkt der Technologiepolitik.

Die Fähigkeit eines Landes zur Innovation ist jedoch nicht einfach mit Forschung und Entwicklung gleichzusetzen. Abschnitt 2 "Spezialisierungsmuster des österreichischen Innovationssystems" thematisiert Österreichs Humanressourcen, die ein zentraler Faktor bei der Hervorbringung und Aufnahme technologischen Wissens sind. In der Tat werden hochqualifizierte und gut ausgebildete Arbeitskräfte zu den wesentlichsten Standortvorteilen Österreichs gezählt. Bei den Naturwissenschaftlern und Ingenieuren, einem hochqualifizierten Segment der Humanressourcen, geht jedoch ein international niedriger Anteil an den Erwerbstätigen mit einer außerordentlich niedrigen Neuabsolventenquote einher. Die Einrichtung eines Fachhochschulsystems läßt eine Erweiterung des Qualifikationsangebots erwarten, die der österreichischen Ausgangslage angepaßt ist. Ein weiterer Aspekt der Spezialisierung von Innovationssystemen ist die Spezialisierung von Patentaktivitäten. Die Ergebnisse der Untersuchung der österreichischen Patentanmeldungen stehen in Einklang mit den Befunden in bezug auf das Forschungsund Entwicklungssystem (relativ geringe Patentintensität und Internationalisierung der Patentaktivitäten). Die Patentspezialisierung Österreichs weist darüber hinaus Besonderheiten auf: Der Schwerpunkt der österreichischen Patentanmeldungen am Europäischen Patentamt liegt im Technologiebereich "Bauwesen" (getragen von innovativen Unternehmen, die in ihren jeweiligen Marktnischen oft eine bedeutende Position auf dem Weltmarkt innehaben), während wichtige Hochtechnologiebereiche wie zum Beispiel Instrumente, Elektronik und Nachrichtentechnik im internationalen Vergleich deutlich unterrepräsentiert sind.

In offenen Wirtschaften, die miteinander durch internationalen Handel, Direktinvestitionen und Informationsströme verflochten sind, hängt die Entwicklung der wirtschaftlichen Leistungsfähigkeit - insbesondere der Produktivität - nicht nur von den eigenen technologischen Anstrengungen eines Landes ab. Die Fähigkeit von Ländern, im Ausland produziertes Wissen aufzunehmen und intelligent zu nutzen wird zu einem entscheidenden Faktor für die Realisierung eines angemessenen Wirtschaftswachstums. Zur Herstellung dieser Fähigkeit bedarf es allerdings zunehmend eigener Anstrengungen in Forschung und Entwicklung. Kernstück von Abschnitt 3 "Internationale Technologieströme" ist eine Analyse des österreichischen Außenhandels nach seinem "technologischen Gehalt". Mit dem Import von Waren und Dienstleistungen wird einerseits technologisches Wissen importiert. Gleichzeitig aber schafft ein hohes technologisches Entwicklungsniveau auch Wettbewerbsvorteile im Export und beeinflußt damit die Höhe der in einer Volkswirtschaft erzielbaren Einkommen. Österreich hat bekanntlich ein strukturelles Defizit im Außenhandel mit Industriewaren. Weniger bekannt ist, daß ein großer Teil dieses Defizits im Handel mit humankapitalintensiven Gütern (darunter auch Hochtechnologie) entsteht. Im österreichischen Außenhandel besteht also nach wie vor eine "Technologielücke". Sowohl in den OECD- als auch in den EU-Ländern entfällt auf Hochtechnologie ein mehr als doppelt so hoher Exportanteil wie in Österreich. Positiv ist zu vermerken, daß ein 
Strukturwandel der österreichischen Exporte zugunsten technologisch anspruchsvollerer Waren im Gang ist und internationale Marktanteilsgewinne erzielt werden konnten. Die aufgrund geänderter komparativer Vorteile vorhersehbare Erhöhung des Außenhandelsdefizits etwa bei arbeitsintensiven Produkten konnte dadurch jedoch nicht kompensiert werden. Die Technologische Zahlungsbilanz, die den internationalen Handel mit technischem Wissen und Dienstleistungen mit technologischem Inhalt wiedergibt, zeigt ein ähnliches Bild. Die entsprechenden Einnahmen deckten zuletzt rund ein Viertel der Ausgaben ab. Dies mag im Zug eines raschen technologischen Aufholprozesses nicht weiter problematisch sein. Mit dieser Deckungsquote liegt Österreich allerdings konstant am unteren Ende der OECD-Länder.

Unter der Diffusion einer Technologie versteht man den Prozeß der Verbreitung einer Technologie vom erstmaligen Einsatz bis zur weitverbreiteten Anwendung. Wie bereits erwähnt spielt die Diffusion von Technologien eine wichtige Rolle für die Produktivitätsentwicklung. Abschnitt 4 "Diffusion von Technologien" behandelt die Diffusion in zwei Technologiebereichen, und zwar in den Informations- und Kommunikationstechnologien sowie in ausgewählten Technologien für eine nachhaltige Entwicklung (Kombinierter Verkehr und Kraft-Wärme-Kopplung). Die Informations- und Kommunikationstechnologie durchdringt als sogenannte generische Technologie zunehmend alle Bereiche der Wirtschaft und Gesellschaft. Österreich konnte im Unterschied zu anderen Ländern mit ursprünglich im Verhältnis zum Bruttoinlandsprodukt geringen Ausgaben für Informationstechnologie im Lauf der letzten Jahre deutlich aufholen und Anschluß an den westeuropäischen Durchschnitt finden. Der Markt für Informationsund Kommunikationstechnologie - insbesondere das Software-Segment - wächst in Österreich deutlich rascher als im EU-Durchschnitt. Der Einsatz von Industrierobotern ist eine wesentliche Komponente der internationalen Tendenz zur flexiblen Automation von Produktionsabläufen. In Österreich hat sich die Industrieroboterintensität im letzten Jahrzehnt deutlich erhöht, wobei sich der Schwerpunkt der Automatisierung zu Unternehmen mit weniger als 200 Beschäftigten verlagert hat. Nachhaltiges Wachstum ist ein Schwerpunkt des Entwurfs für das 5. Rahmenprogramm für Forschung und Technologische Entwicklung der EU. Anders als die Informations- und Kommunikationstechnologien hat die Diffusion von Technologien für eine nachhaltige Entwicklung mit einer Reihe von Problemen bei den Rahmenbedingungen zu kämpfen. Dies wird anhand zweier für Österreich relevanter Beispiele - Kombinierter Verkehr und Kraft-Wärme-Kopplung - gezeigt.

Die Einbindung der österreichischen Forschung und Entwicklung in den größeren europäischen Zusammenhang ist eine der zentralen Herausforderungen für die Forschung und Technologiepolitik. Der Spezialteil: "Österreich und die Rahmenprogramme der EU" in Abschnitt 5 faßt die Ergebnisse der ersten Evaluierung der österreichischen Beteiligung an den europäischen Programmen für Forschung und Technologische Entwicklung zusammen. Diese Evaluierung brachte eine Fülle von neuen Erkenntnissen. Positiv bewertet wird dabei, daß die europäischen Programme die strategischen Möglichkeiten der teilnehmenden Unternehmen, Universitätsinstitute und Forschungseinrichtungen deutlich erweitern. Die Teilnahmeintensität Österreichs war zunächst noch vergleichsweise mäßig 
und die Teilnahme durch eine Dominanz der Forschungsinstitutionen gegenüber dem Unternehmenssektor gekennzeichnet. Mit dem EU-Beitritt Österreichs kam es zu einem raschen Aufholprozeß bei der Programmbeteiligung. Die nun vorliegenden Informationen über die österreichische Teilnahme am 4. Rahmenprogramm bestätigen dies und geben Anlaß zu Optimismus. Die Ergebnisse bezüglich der Voraussetzungen für die Teilnahme (etwa bereits existierende Erfahrungen mit F\&E-Kooperationen), der dabei wahrgenommenen Barrieren usw. führen unter anderem zu Schlußfolgerungen zur besseren Auschöpfung des Teilnehmerpotentials und der Nutzung der Ergebnisse. 


\section{Das österreichische Forschungssystem im internationalen Vergleich}

\subsection{Niveau und Struktur der Aufwendungen für Forschung und Entwicklung}

Die Aufwendungen für Forschung und Entwicklung (F\&E) werden seit Jahrzehnten als zentraler Indikator zur Beobachtung des Niveaus der F\&E-Aktivitäten von Volkswirtschaften herangezogen. Über die Forschungsquote, d. h. den Anteil der F\&E-Aufwendungen am Bruttoinlandsprodukt (BIP) werden Rangfolgen erstellt und so die Länder verglichen und bewertet. Dabei zeigen sich zwischen den OECD-Ländern erhebliche Unterschiede sowohl im Niveau als auch in der Entwicklung der Forschungsquoten (siehe Abbildung 1.1.1).

Österreichs Forschungsquote stagniert seit Anfang der neunziger Jahre auf einem Niveau von rund 1,5\%. Der Abstand zum zuletzt leicht fallenden Durchschnitt der OECD und der EU (1995 2,15\% bzw. 1,85\%) hat sich zwar etwas verringert, Österreichs Investitionen in Forschung und Entwicklung sind jedoch nach wie vor gering für ein Land seines Pro-Kopf-Einkommensniveaus.

Ein einfacher internationaler Vergleich der Forschungsquoten hat natürlich nur begrenzte Aussagekraft. Zum einen handelt es sich bei den F\&E-Aufwendungen zunächst um Investitionen, also um Kosten, über deren Erträge nichts aussagt wird. Vor allem aber deckt die aggregierte Betrachtung jene Zusammenhänge zu, die seit etwa einem Jahrzehnt unter dem Begriff "Nationales Innovationssystem"' in Diskussion stehen. Dabei werden nicht so sehr die aggregierten F\&E-Ressourcen betrachtet, sondern die Beziehungen und Verflechtungen zwischen den einzelnen Elementen des jeweiligen Innovationssystems in den Vordergrund gerückt.

Zu diesem Zweck wird auf Basis der aktuellsten Wissenschafts- und Forschungsstatistik der OECD eine detailliertere Darstellung der nationalen F\&E-Strukturen vorgenommen. Die Analyse konzentriert sich im wesentlichen auf die Beziehungen zwischen den F\&Efinanzierenden und F\&E-durchführenden Sektoren. Die Strukturanalysen wurden auf Basis von Daten für das Jahr 1993 durchgeführt, da für Österreich keine aktuelleren Daten vorliegen. Bevor die entsprechenden Ergebnisse vorgestellt werden, seien die wichtigsten Aussagen zu den gesamtwirtschaftlichen F\&E-Aufwendungen getroffen. Als Basis dafür dient Abbildung 1.1.2, die Forschungsquote und BIP pro Kopf 1995 zueinander in Beziehung setzt.

- $\quad$ Es gibt einen - nicht allzu starken - positiven Zusammenhang zwischen Bruttoinlandsprodukt (BIP) pro Kopf und dem Anteil der F\&E-Aufwendungen am BIP.

- Österreich zählt zur Gruppe der reicheren Länder, bleibt mit seiner - seit einigen Jahren stagnierenden - Forschungsquote von rund 1,5\% aber zurück und befindet sich in der Gruppe der reicheren Länder an letzter Stelle. Nur wenige Länder liegen unter dieser Marke, nämlich Italien, Irland, Spanien und Griechenland. 
- Erst mit einer Anhebung der F\&E-Aufwendungen von 1,5\% auf über $2 \%$ würde Österreich in die Ländergruppe mit sowohl überdurchschnittlichem Einkommen als auch einer (gemessen am Median) überdurchschnittlichen Forschungsquote eintreten.

- Daß dies im Prinzip möglich und politisch durchsetzbar ist, demonstriert gegenwärtig Finnland, wo eine konsequente Politik darauf ausgerichtet ist, daß die Forschungsquote innerhalb von drei Jahren von $2,4 \%$ auf $2,9 \%$ steigt. Bereits in den achtziger Jahren konnte Finnland einen unter den OECD-Ländern beispiellosen technologischen Aufholprozeß realisieren. Es sei in diesem Zusammenhang daran erinnert, daß Finnland und Österreich zu Beginn der achtziger Jahre von einem identischen Niveau der Forschungsquote aus starteten. Unter den Ländern mit steigender Forschungsquote befindet sich auch Dänemark.

Abbildung 1.1.1: Entwicklung der Forschungsquoten (F\&E-Ausgaben in Prozent des BIP) in ausgewählten Ländern, 1991,1993,1995

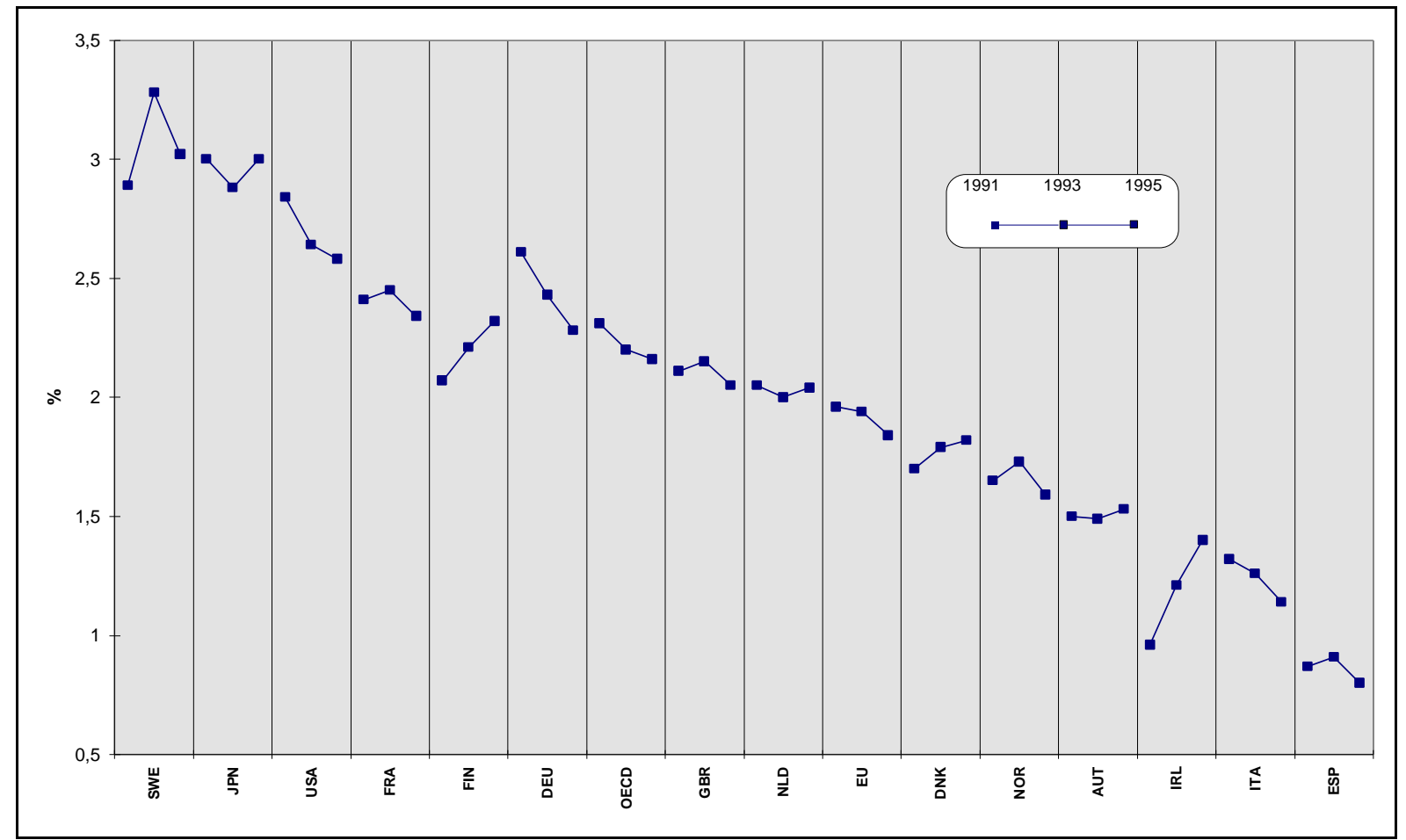

Q: OECD-MSTI.

Entgegen dem internationalen Trend haben Irland und Finnland ihre Forschungsquoten deutlich erhöht. Diesen Ländern ist es in den letzten Jahren gelungen eindrucksvoll aufzuholen bzw. in das internationale Spitzenfeld vorzudringen. Auch Dänemark konnte seine Forschungsquote nachhaltig steigern. 
Abbildung 1.1.2: Forschungsquote und BIP pro Kopf nach Ländern, 1995

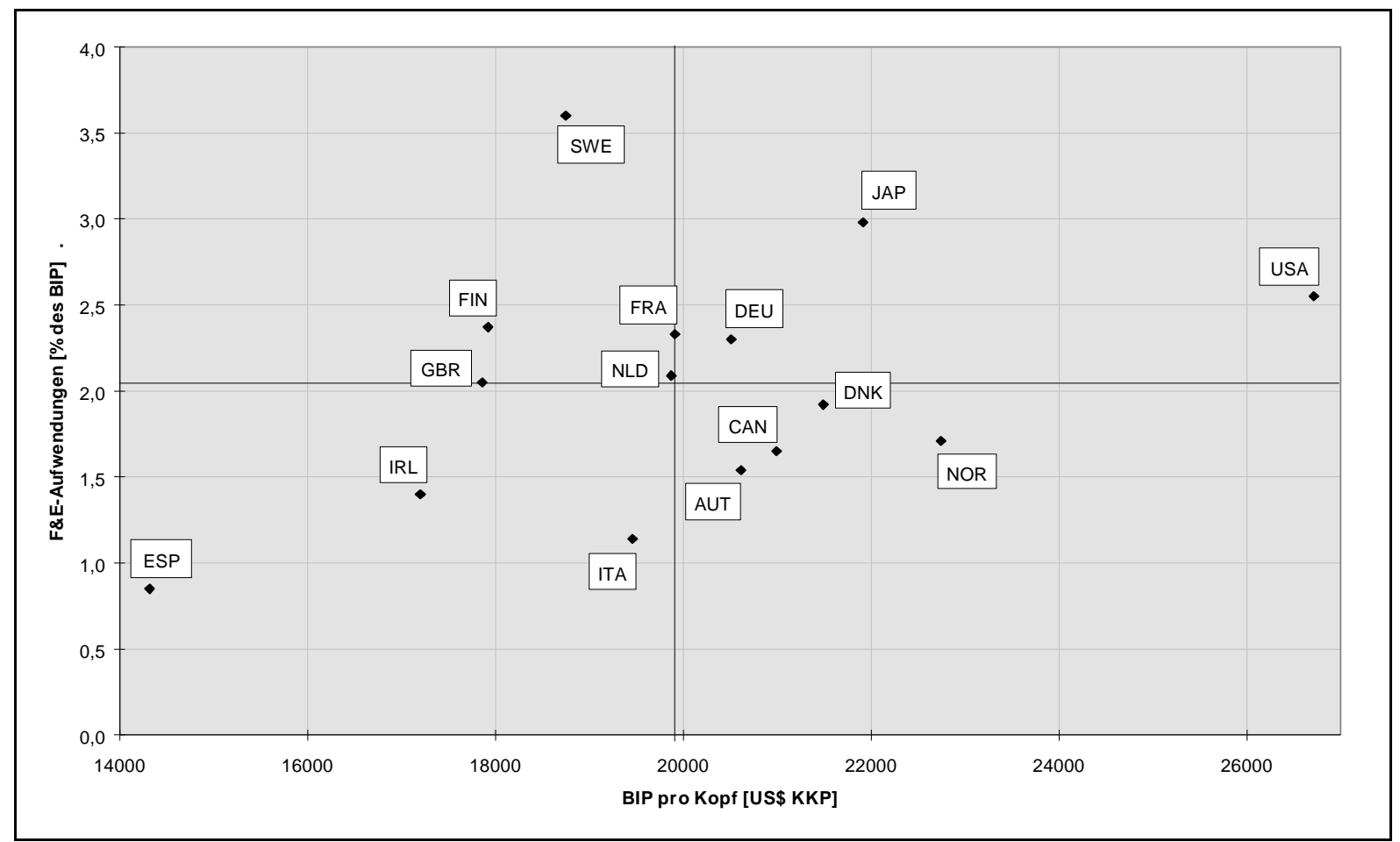

Q: OECD-MSTI, ÖSTAT.

Ein einfacher Vergleich der Forschungsquoten berücksichtigt auch nicht, daß es zwischen den Ländern erhebliche Unterschiede in der Verteilung der F\&E-Aufwendungen auf die Sektoren gibt, die die F\&E-Leistungen ausführen. In Abbildung 1.1.3 werden vier Sektoren unterschieden, die in Österreich folgendermaßen abgegrenzt sind:

Unternehmenssektor, einschließlich Österreichisches Forschungszentrum Seibersdorf und Joanneum Research,

öffentlicher Sektor, einschließlich Bundesinstitutionen (unter Ausklammerung der im Hochschulsektor zusammengefaßten), Landes-, Gemeinde-, Kammerinstitutionen sowie Einrichtungen von Sozialversicherungsträgern, Museen und Krankenanstalten),

Hochschulsektor, einschließlich Universitätskliniken, Kunsthochschulen, Österreichische Akademie der Wissenschaften sowie Versuchsanstalten an HTLs, sowie

privater gemeinnütziger Sektor, der private Institutionen ohne Erwerbscharakter, mit vorwiegend privatem oder privatrechtlichem, konfessionellem oder sonstigem nicht öffentlichen Status umfaßt. 
Innovation ist eine treibende Kraft des Wirtschaftswachstums. Forschung und Entwicklung wird in den entwickelten Volkswirtschaften primär im Unternehmenssektor durchgeführt. Besonders deutlich ausgeprägt ist diese Tendenz in Ländern mit hoher Forschungsquote. In Österreich spielt der Unternehmenssektor eine relativ geringere Rolle.

Betrachtet man die OECD-Länder im Vergleich und positioniert im speziellen Österreich darin, so ergibt sich folgendes Bild:

- Die Verteilung der F\&E-ausführenden Sektoren variiert stark von Land zu Land. Typischerweise nimmt der Unternehmenssektor den größten Anteil in der Durchführung von F\&E ein (mit Ausnahme Griechenlands, wo der Universitätssektor dominiert). In allen Ländern außer Griechenland (mit 27\%) und Spanien (mit 48\%) führt der Unternehmenssektor sogar mehr als die Hälfte der gesamtwirtschaftlichen F\&E aus. Österreich rangiert mit einem Unternehmensanteil von $56 \%$ im unteren Drittel der OECD-Länder.

- Aus Abbildung 1.1.3 läßt sich die generelle Tendenz ablesen, daß forschungsintensivere Länder einen höheren Unternehmensanteil aufweisen. In den forschungsintensivsten Ländern (Schweden, Japan, USA, Deutschland, Frankreich, Großbritannien und Finnland) führt der Unternehmenssektor $2 / 3$ bis $3 / 4$ der gesamtwirtschaftlichen F\&E-Leistungen durch.

- In Österreich belaufen sich die vom Unternehmenssektor durchgeführten F\&ELeistungen auf $0,86 \%$ des BIP (1993) gegenüber 1,46\% bzw. 1,15\% im OECD- bzw. EU-Durchschnitt. Dieser Betrag setzt sich zusammen aus den eigenfinanzierten Aufwendungen des Unternehmenssektors (0,74\% bzw. 15,527 Mrd. S), der Finanzierung durch den öffentlichen Sektor ("Forschungsförderung") in Höhe von $0,08 \%$ des BIP bzw. 1,738 Mrd. S sowie aus der Finanzierung aus dem Ausland (0,04\% des BIP bzw. 0,82 Mrd. S).

- Einen relativ hohen Anteil hat in Österreich der Hochschulsektor mit 0,53\% des BIP. Dem gegenüber ist die im öffentlichen bzw. im privaten gemeinnützigen Sektor durchgeführte F\&E in ihrem Umfang ausgesprochen bescheiden. Im Unterschied dazu erreichen die im öffentlichen Sektor erstellten F\&E-Leistungen in vielen Ländern dieselbe Größenordnung wie die im Hochschulsektor erbrachten.

- Die auffallend geringe Präsenz des öffentlichen Sektors in Österreich ist nicht zuletzt darauf zurückzuführen, daß das Österreichische Forschungszentrum Seibersdorf und Joanneum Research - entsprechend den geltenden OECD-Richtlinien ${ }^{1}$ ) - dem Unternehmenssektor zugerechnet werden.

$\left.{ }^{1}\right)$ Siehe $\underline{O E C D}$ (1993). Die Konvention über den Erhebungsmodus von F\&E-Aktivitäten sieht vor, gesellschaftsrechtlich organisierte F\&E-Einrichtungen, deren Umsatzerlöse aus der Auftragsforschung und aus der Projektförderung 50\% der Gesamtausgaben überschreiten, dem Unternehmenssektor zuzurechnen. 
Der Vergleich der Strukturen nach ausführenden Sektoren mit jenen nach finanzierenden Sektoren liefert Hinweise auf den Grad der Verflechtung zwischen den einzelnen Sektoren innerhalb der nationalen Forschungs- und Innovationssysteme bzw. deren Grad der Internationalisierung. Der Grad der Verflechtung ist um so höher, je mehr die F\&EAufwendungen eines Sektors von anderen Sektoren finanziert werden.

Die öffentliche Finanzierung von F\&E ist generell höher als die Ausführung im staatlichen Sektor. In den meisten Ländern liegt die öffentliche Finanzierung in einer Bandbreite von 0,7-1\% des BIP. Die großen Unterschiede in den nationalen Forschungsquoten sind auf unterschiedlich starkes Engagement des Unternehmenssektors bzw. auf Auslandsfinanzierung zurückzuführen. Während die Finanzierung von F\&E durch den öffentlichen Sektor in Österreich mit 0,73\% des BIP genau dem OECD-Durchschnitt entspricht, ist der Unternehmensanteil deutlich unterdurchschnittlich.

Positioniert man Österreich im internationalen Rahmen, so lassen sich im Detail folgende Aussagen treffen (siehe vor allem Abbildung 1.1.4):

- Generell ist die öffentliche Finanzierung von F\&E deutlich höher als die Ausführung von F\&E im staatlichen Sektor (Hochschulen und Forschungseinrichtungen im öffentlichen Sektor).

- In manchen Ländern liegt der Finanzierungsanteil des öffentlichen Sektors in der selben Größenordnung wie jener der Industrie. Dies gilt insbesondere für Österreich, Kanada, Spanien, Italien, Niederlande und Norwegen. In Österreich betrug der öffentliche Finanzierungsanteil an den gesamtwirtschaftlichen F\&E-Ausgaben 1995 49,1\%. Dies ist der höchste Anteil unter den hochentwickelten europäischen OECDLändern. Nach jüngsten Schätzungen soll dieser Anteil 1997 auf 46,5\% zurückgehen.

Im Jahr 1995 betrug der öffentliche Finanzierungsanteil an den gesamtwirtschaftlichen F\&E-Ausgaben in Österreich 49,1\%. Dies ist der höchste Anteil unter den hochentwickelten europäischen OECD-Ländern.

- Was die Finanzierung durch das Ausland betrifft, so läßt sich daran nur ein geringer Grad an Internationalisierung bzw. Globalisierung der F\&E-Tätigkeit erkennen. Die Finanzierung aus dem Ausland ist freilich nur ein Aspekt der Internationalisierung von Forschung und Entwicklung. In den meisten "alten" EU-Ländern läßt sich ein etwas höheres Niveau der ausländischen Finanzierung von F\&E erkennen: Dies ist zu nicht unerheblichen Teilen auf die EU-Programme für Forschung und technologische Entwicklung zurückzuführen. Es ist daher zu erwarten, daß sich in den neuen Mitgliedsländern Finnland, Schweden und Österreich der Anteil der ausländischen F\&EFinanzierung in Zukunft erhöhen wird. Zumindest auf Basis der Daten zur internationalen Forschungsfinanzierung kann nur sehr eingeschränkt von einem global sourcing im F\&E-Bereich gesprochen werden. Für die EU insgesamt betrug der Anteil der 
auslandsfinanzierten F\&E 1995 6,5\%. Mit einem Anteil von 2,5\% (bzw. 2,6\% in den Jahren 1996 und 1997) liegt Österreich noch deutlich unter dem Durchschnitt der EU und hinter anderen kleinen offenen Volkswirtschaften zurück (z.B. Dänemark 9,9\%, Finnland 4,5\%, Irland 8,2\%, Niederlande 9,3\%, Norwegen 4,9\%).

Mit einem Anteil der Finanzierung aus dem Ausland an den gesamtwirtschaftlichen F\&E-Ausgaben von 2,5\% (1995) liegt Österreich deutlich unter dem Durchschnitt der EU $(6,5 \%)$ und hinter anderen kleinen offenen Volkswirtschaften in Europa.

Ein besonders wichtiger Indikator für die Charakterisierung nationaler Innovationssysteme ist das Verhältnis von öffentlicher und unternehmensseitiger Finanzierung von F\&E. Abbildung 1.1.5, in der die Niveaus der F\&E-Finanzierung durch die beiden Sektoren (in Prozent des BIP) einander gegenübergestellt sind, liefert die Grundlage für einige interessante Beobachtungen:

- Ein hohes Niveau der F\&E-Finanzierung durch den Unternehmenssektor geht in der Regel mit einem hohen Niveau der F\&E-Finanzierung durch den öffentlichen Sektor einher - ohne hier eine Aussage über die Kausalität treffen zu wollen. Japan und in geringerem $\mathrm{Ma}$ Schweden sind durch die Dominanz unternehmensfinanzierter F\&E gekennzeichnet.

- Österreich wird oft mit anderen hochentwickelten kleinen offenen Volkswirtschaften verglichen, darunter auch Finnland und Schweden. Für die Wahl dieser Referenzländer gibt es gute Gründe. Gleichzeitig geht aus Abbildung 1.1.5 klar hervor, daß - was die Finanzierungsstruktur des nationalen F\&E-Systems betrifft - Österreich der Ländergruppe bestehend aus Dänemark, den Niederlanden, Norwegen, Kanada und Italien sehr viel näher steht als etwa Schweden und Finnland.

- Während die Finanzierung von F\&E durch den öffentliche Sektor in Österreich mit 0,73\% des BIP genau dem Durchschnitt (Median) der OECD-Staaten entspricht, bleibt der Unternehmensanteil deutlich unterdurchschnittlich. Eine Erhöhung der staatlichen Finanzierung von F\&E im Unternehmenssektor sollte daher strikt an die nachhaltige Erhöhung der unternehmensfinanzierten F\&E gebunden werden. 
Abbildung 1.1.3: Aufwendungen für F\&E nach ausführenden Sektoren, 1993

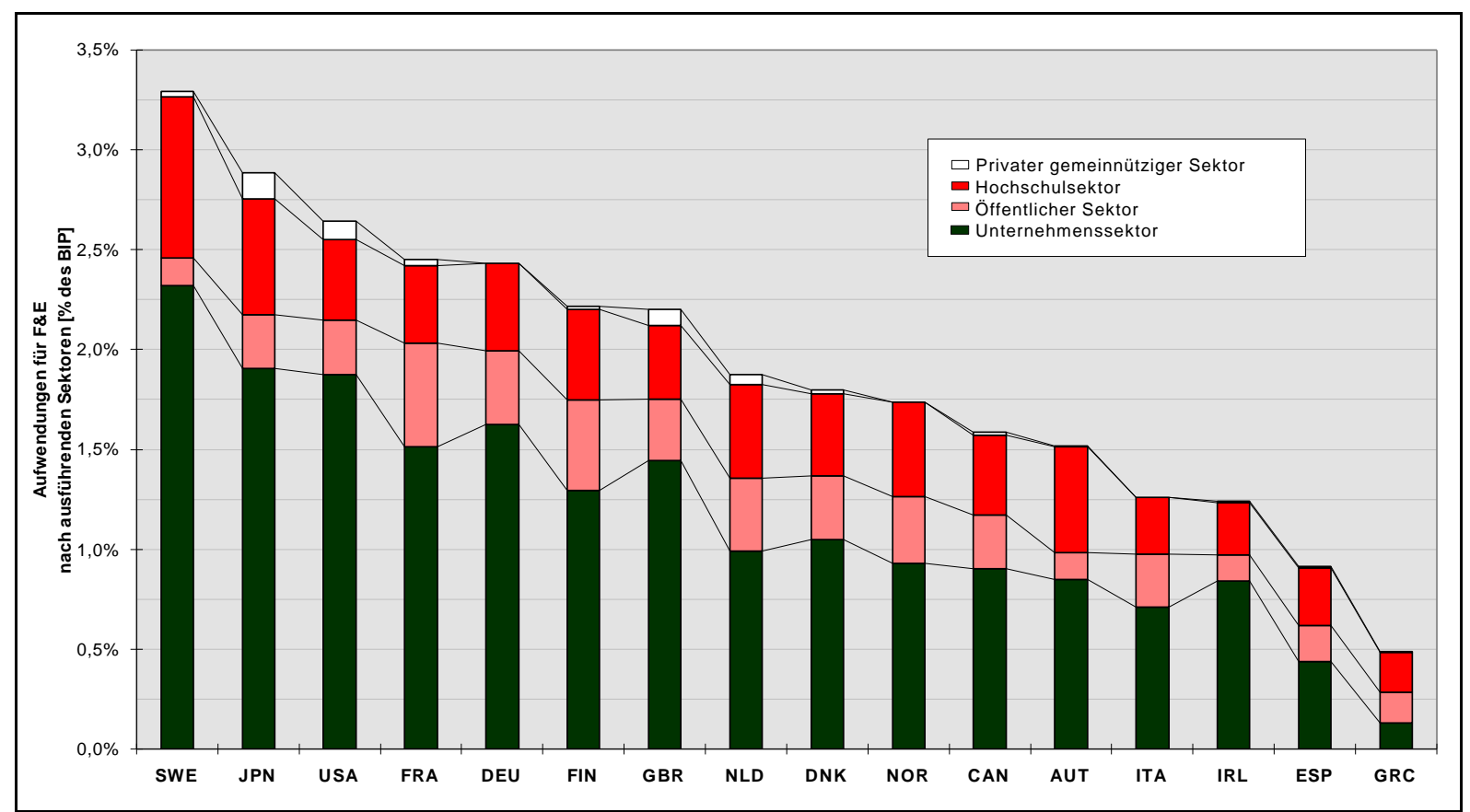

Q: OECD-MSTI, ÖSTAT.

Abbildung 1.1.4: Aufwendungen für F\&E nach finanzierenden Sektoren, 1993

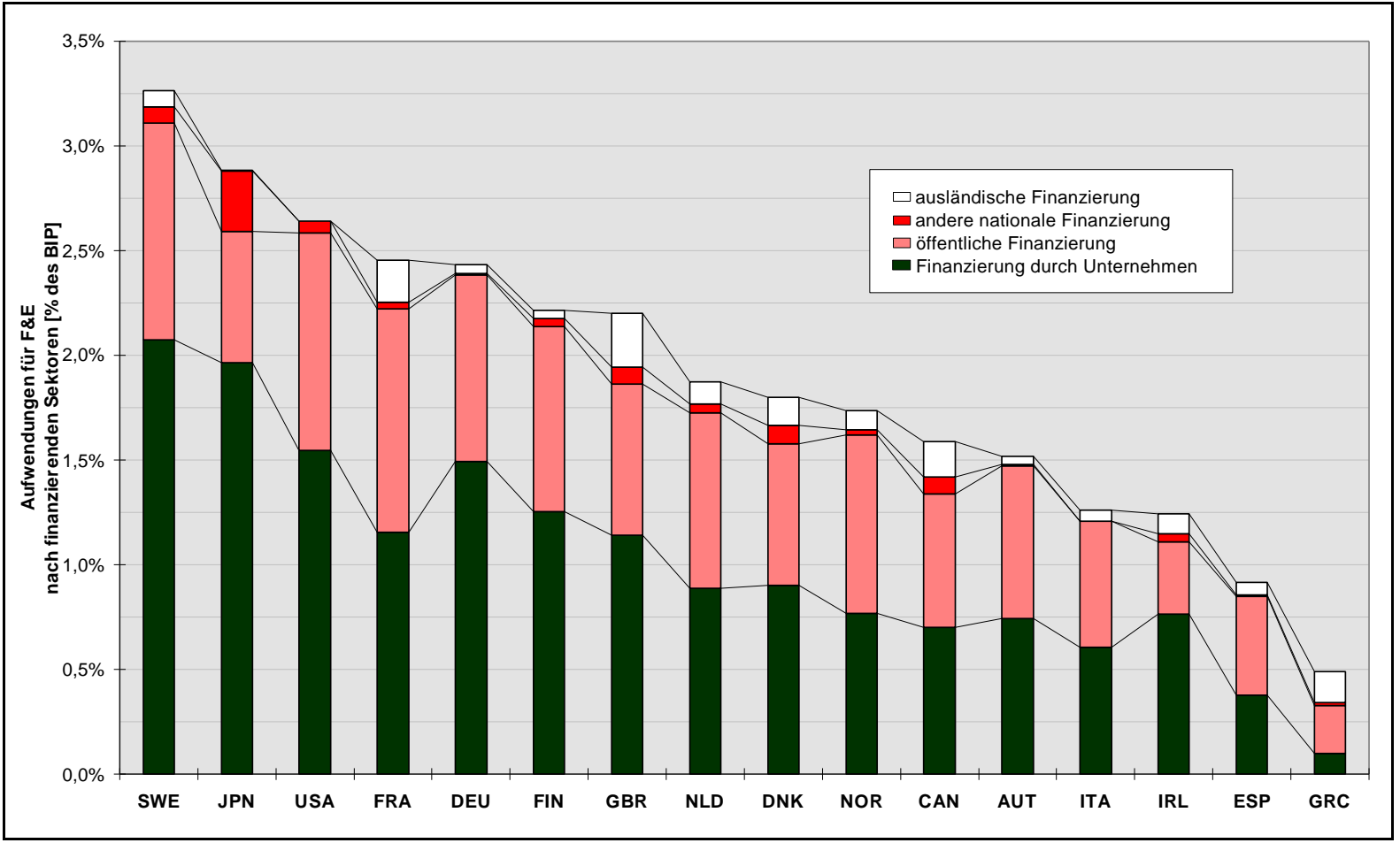

Q: OECD-MSTI, ÖSTAT. 


\section{$1.2 \quad$ Finanzierung der Industrieforschung}

Der überwiegende Teil der in Unternehmen durchgeführten F\&E wurde 1993 in allen Vergleichsländern durch den Unternehmenssektor selbst finanziert. Mit einem eigenfinanzierten Anteil der Unternehmens-F\&E von 86\% befindet sich Österreich in einer Gruppe mit Dänemark, Irland, Schweden, Deutschland und Finnland. Am niedrigsten ist dieser Anteil mit $70-80 \%$ in großen Ländern mit hohen Rüstungsausgaben, wie in Großbritannien, Frankreich und den USA. Nähere Betrachtung verdient jedoch vor allem die Verschränkung des Unternehmenssektors mit anderen Sektoren hinsichtlich Finanzierung und Ausführung von F\&E.

Die in Unternehmen durchgeführte F\&E wird zum Großteil vom Unternehmenssektor selbst finanziert. Der Anteil der öffentlichen Finanzierung von F\&E im

Unternehmenssektor - in Form direkter Forschungs- und Technologieförderung überschreitet 10\% nur in Ländern mit hohen Rüstungsausgaben. In Österreich betrug der Anteil 1993 9,8\%.

Die gemeinhin unter dem Titel "direkte Forschungs- und Technologieförderung" stattfindende öffentliche Finanzierung von F\&E im Unternehmenssektor nimmt eine zentrale Rolle in der forschungs- und technologiepolitischen Diskussion ein. Die folgenden Ausführungen beleuchten dieses Thema und vergleichen Österreich mit den anderen OECD-Ländern (vgl. Abbildung 1.2.1):

- In Österreich fließen $11 \%$ der öffentlichen F\&E-Mittel an den Unternehmenssektor. Damit werden $9,8 \%$ der von den Unternehmen ausgeführten F\&E durch die öffentliche Hand finanziert. Österreich liegt mit beiden Werten im internationalen Mittelfeld. Die direkte Forschungsförderung lag damit 1993 auf internationalem Niveau.

- Im internationalen Spitzenfeld staatlicher Finanzierung unternehmerischer F\&E liegen rüstungsforschungsintensive Länder wie die USA, Frankreich und Großbritannien sowie, als Ausnahme von dieser Regel, Irland. In Norwegen ist der hohe Anteil durch einen hohen Anteil an öffentlich finanzierter F\&E im Bereich der Energie (Erdöl und Erdgas) begründet.

- Umgekehrt fällt auf, daß in einer Reihe von Ländern das Verhältnis zwischen öffentlichem und Unternehmenssektor, gemessen an der öffentlichen Finanzierung privat ausgeführter F\&E weitgehend entkoppelt ist. Dies darf - mit Verweis auf Japan nicht als generelle Absenz des Staates interpretiert werden, sondern vielmehr als Beleg dafür, daß staatliches Handeln nicht auf öffentliche Finanzierung beschränkt ist.

Zusätzliche wertvolle Informationen zur Entwicklung der Technologieförderung in Österreich bietet das beim Bundeskanzleramt eingerichtete Dokumentationssystem Finkord. Die 
Finkord-Daten belegen, daß die technologiebezogene Wirtschaftsförderung ${ }^{2}$ ) des Bundes bis zur Mitte der neunziger Jahren zügig ausgeweitet wurde.

Abbildung 1.1.5: Finanzierung von F\&E durch den öffentlichen und den Unternehmenssektor, 1993

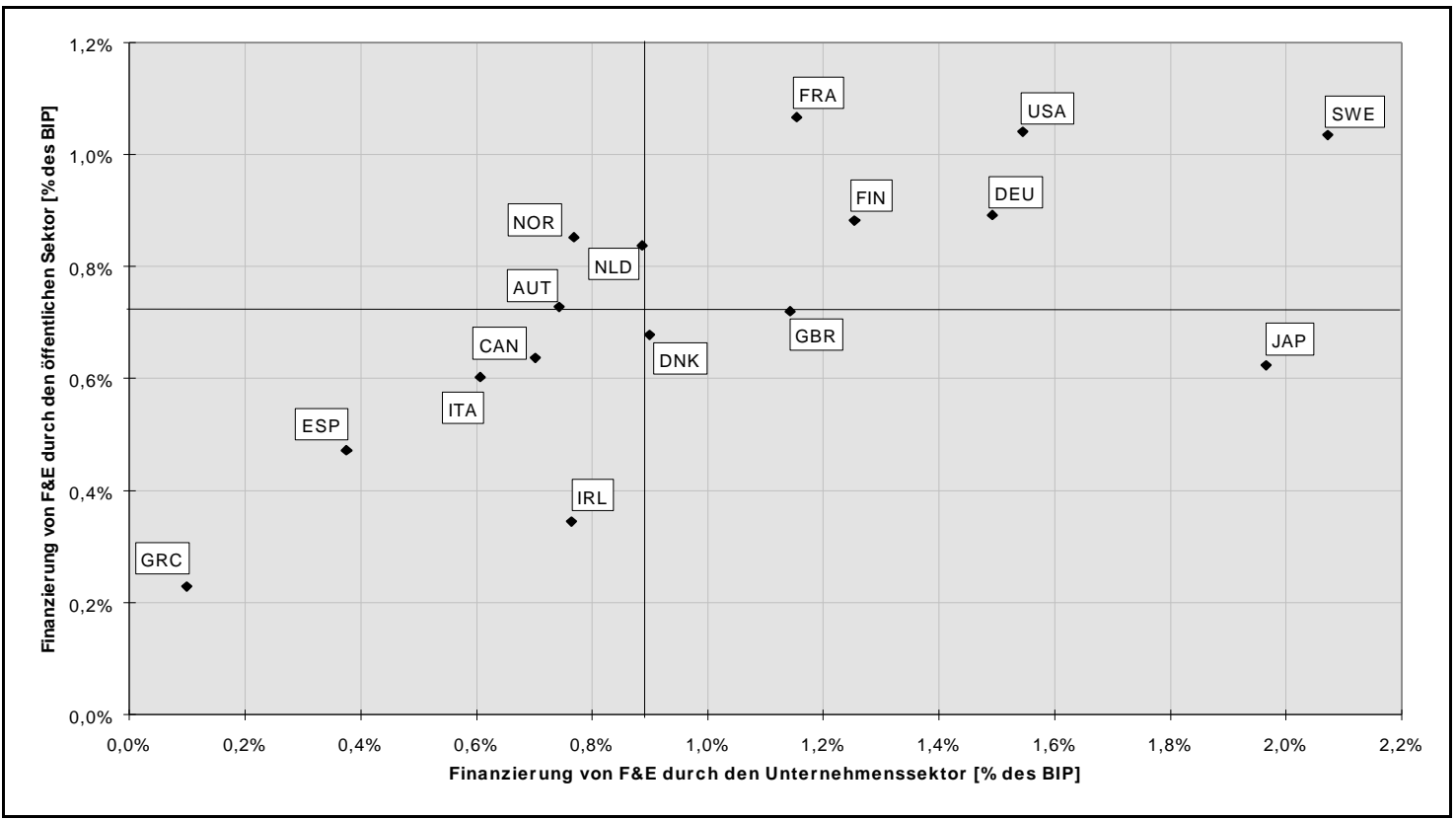

Q: OECD-MSTI, ÖSTAT.

${ }^{2}$ ) Finkord erfaßt die unternehmensbezogene Wirtschaftsförderung des Bundes. Folgende Förderprogramme werden dabei dem Technologiebereich zugerechnet: ITF, FFF, ERP-Innovations- und Technologieförderung, TOP-Fertigungsüberleitung, ERP-Infrastrukturprogramm (Technologiezentren), Struktur- und Qualitätsoffensivprogramm des BMWV, EU-Kofinanzierungen. Die Technologieförderung laut Finkord ist breiter definiert als F\&E-Förderung. 
Abbildung 1.2.1: Öffentliche Finanzierung der Unternehmens-F\&E, 1993

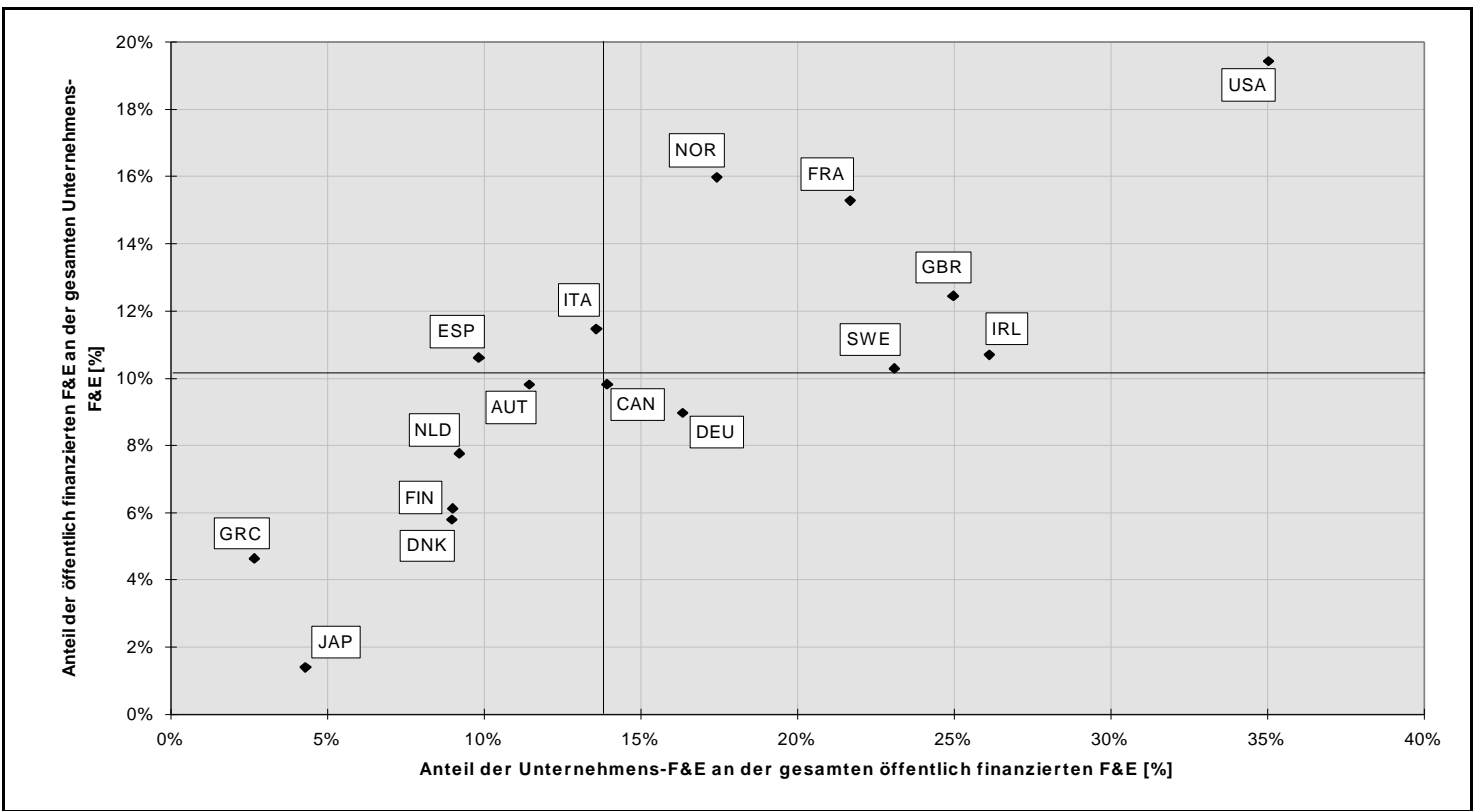

Q: OECD-MSTI, ÖSTAT.

Abbildung 1.2.2: Unternehmensbezogene Wirtschaftsförderung des Bundes Barwert in Mill. $S$

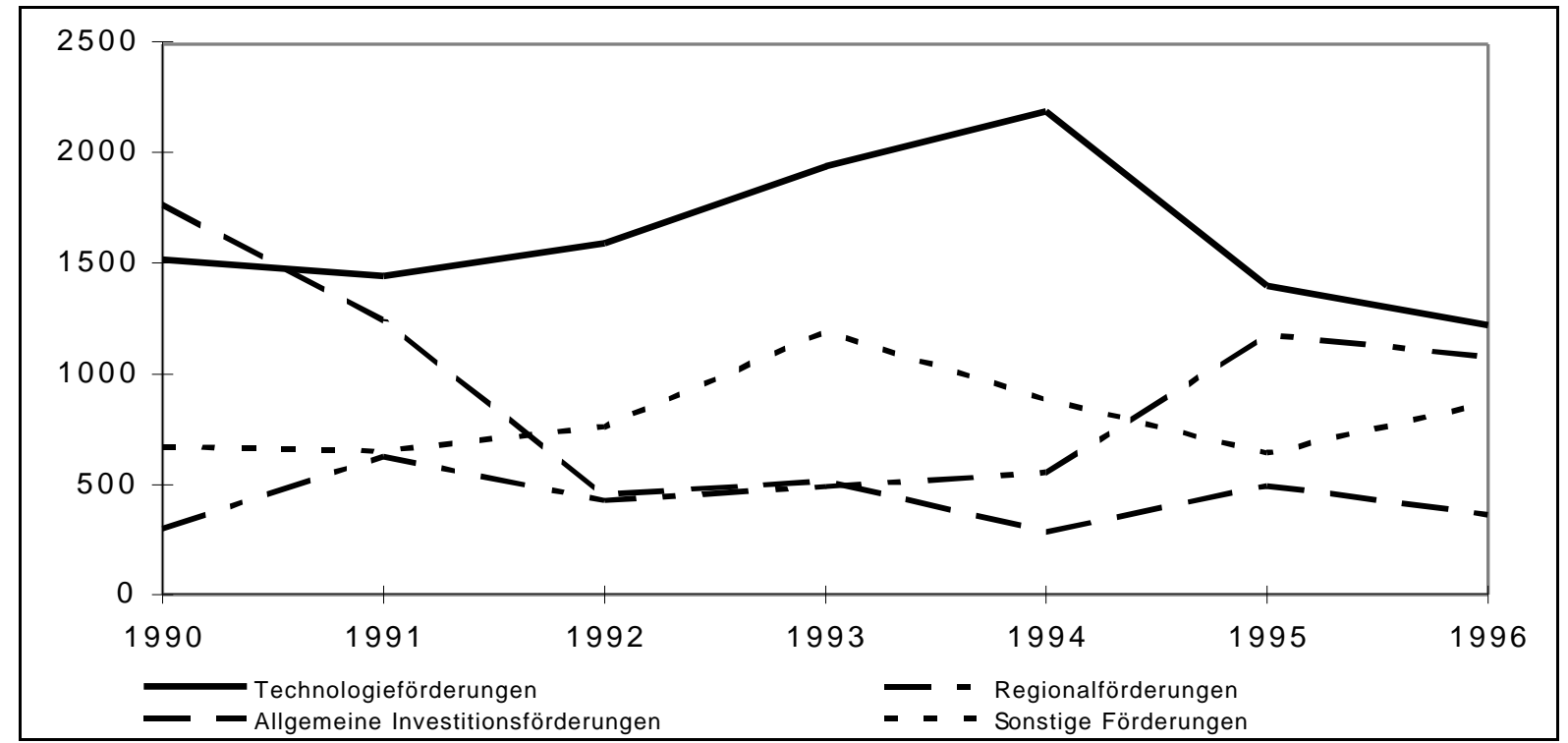

Q: BKA (Finkord). 
Abbildung 1.2.3: Anteil der Technologieförderung am Barwert der unternehmensbezogenen Wirtschaftsförderung des Bundes

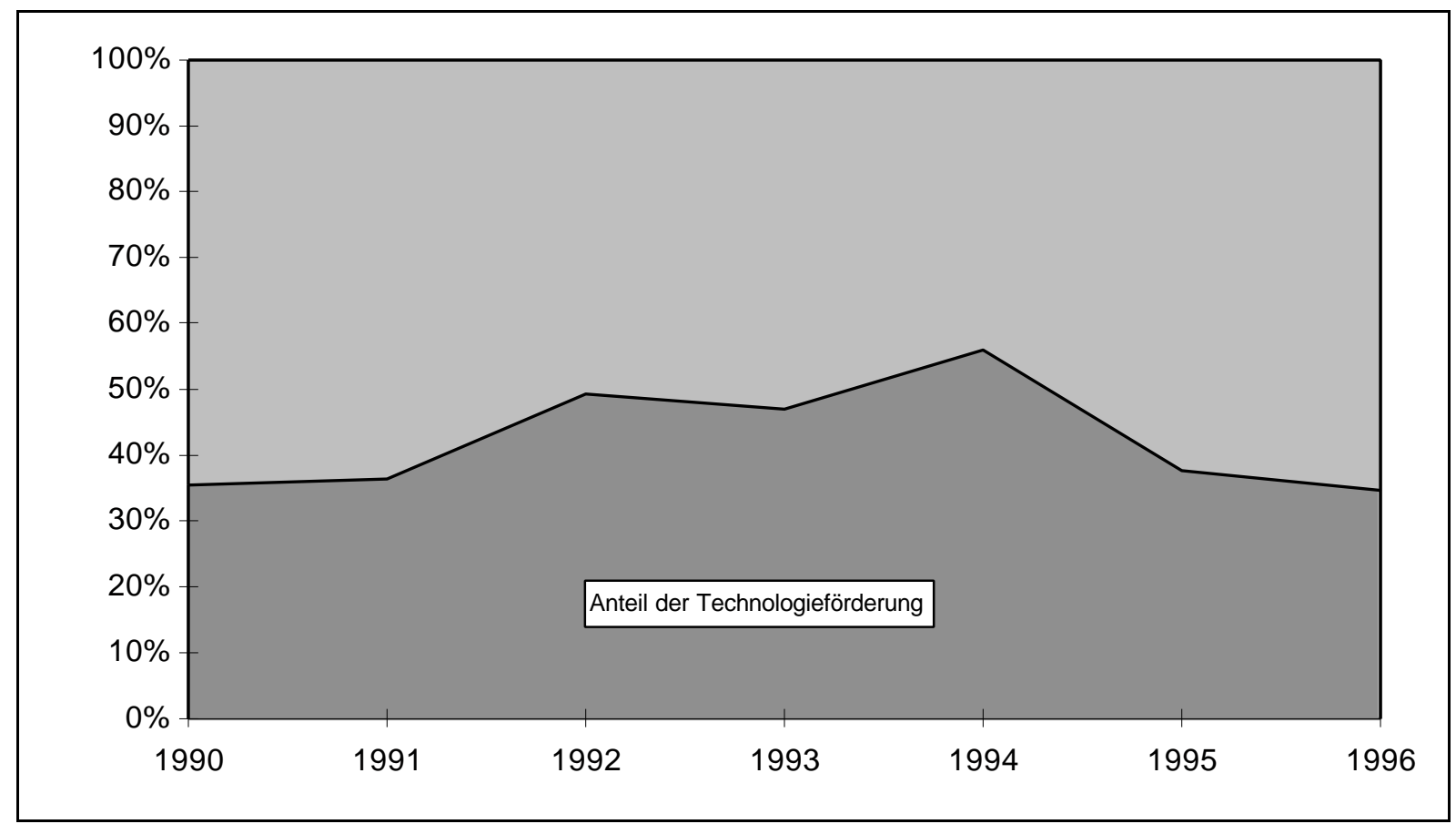

Q: BKA (Finkord).

Mitte der neunziger Jahre geht in Österreich die direkte Technologieförderung durch den Bund drastisch zurück. Im Jahr 1995 betrug die Kürzung 36\% und 1996 weitere $14 \%$.

Gemäß Finkord war bis zum Jahr 1994 ein kontinuierliches Wachstum des Barwerts der Technologieförderung auf 2,2 Mrd. S festzustellen (siehe Abbildung 1.2.2). In den Jahren 1995 und 1996 gingen die Förderungsbarwerte jedoch drastisch (auf 1,4 bzw. auf 1,2 Mrd. S) zurück. Der Anteil der technologiebezogenen Wirtschaftsförderung am Barwert der gesamten unternehmensbezogenen Wirtschaftsförderung des Bundes ist ebenfalls rückläufig und bewegt sich 1995 und 1996 etwa auf dem Niveau der frühen neunziger Jahre (siehe Abbildung 1.2.3). Nach Angaben des Bundeskanzleramts ist der Trendbruch 1995 auf Kürzungen beim ITF (-272 Mill. S), im Bereich des ERP-Fonds (-298 Mill. S) und durch das Auslaufen der TOP-Aktion (-162 Mill. S) zurückzuführen. 1996 kam es zu einer weiteren Reduktion beim ITF (-130 Mill. S) und beim ERP-Fonds ( -75 Mill. S). Die Rückgänge beim ERP-Technologieprogramm sind durch eine Umschichtung der Mittel zum Bereich Regionalförderung zu erklären (+480 Mill. S). Dies ist insbesondere auf die Erfordernisse der Kofinanzierung von EU-Förderungen zurückzuführen. Allerdings deutet manches darauf hin, daß der Innovationsgehalt der außerhalb der "technologiebezogenen Wirtschaftsförderung" geförderten Projekte zugenommen hat. 
Die Finanzierung der Unternehmens-F\&E durch das Ausland gibt einen Hinweis auf den Grad der Globalisierung eines Landes als Anbieter von F\&E-Leistungen ( $g$ lobal sourcing im F\&E-Bereich).

Trotz bescheidener Finanzierung von Unternehmens-F\&E durch das Ausland fließen in Österreich mehr als 90\% der ausländischen F\&E-Mittel an Unternehmen. Dies illustriert den noch relativ geringen Internationalisierungsgrad der gesamten österreichischen Forschung.

- Wie Abbildung 1.2.4 zeigt, liegt der Anteil der Finanzierung der Unternehmens-F\&E durch das Ausland im Schnitt (Median) bei 7,5\%.

- Starke Abweichungen gibt es im Fall von Griechenland, Kanada und Großbritannien, wo ein verhältnismäßig hoher Anteil der privat durchgeführten F\&E aus dem Ausland finanziert wird (zwischen 15 und 27\%).

- Österreich befindet sich mit seiner Auslandsfinanzierung in einer seltsamen Gesellschaft mit den zwei forschungsintensivsten Ländern der Welt, Schweden und Japan. Gemeinsam ist dieser Gruppe der hohe Anteil an der ausländischen Finanzierung, der an die Industrie geht (über $80 \%$ ) und zweitens der geringe Anteil an der Unternehmensfinanzierung durch das Ausland insgesamt. Japan ist in dieser Hinsicht als ein Sonderfall zu betrachten. Was den Vergleich mit Schweden betrifft, so ist der Unterschied zu Österreich auf das Ausmaß der privaten F\&E in beiden Ländern zurückzuführen: 0,85\% Anteil am BIP des privaten Sektors in Österreich gegenüber $2,3 \%$ in Schweden.

- Österreichische Unternehmen ${ }^{3}$ ) beziehen knapp 4\% ihrer F\&E-Mittel aus dem Ausland und nehmen $91 \%$ der gesamten F\&E-Mittel aus dem Ausland für sich in Anspruch.

- Diesem hohen Anteil der ausländischen Finanzierung von F\&E, der an die Industrie geht, steht eine geringe Auslandsfinanzierung von F\&E außerhalb des Unternehmenssektors gegenüber. Dies deutet auf einen insgesamt geringen Internationalisierungsgrad der österreichischen Forschung hin, insbesondere aber auf eine weitgehende Absenz des österreichischen Hochschulsektors vom internationalen Forschungsmarkt. Es ist allerdings davon auszugehen, daß im Zuge der nunmehr massiven Teilnahme Österreichs an den Rahmenprogrammen der Europäischen Union für Forschung und technologische Entwicklung diese Lücke verringert wird.

\footnotetext{
$\left.{ }^{3}\right)$ Wie oben erläutert, werden die beiden größten außeruniversitären Forschungseinrichtungen Forschungszentrum Seibersdorf und die Joanneum Research GmbH - in der amtlichen Statistik dem Unternehmenssektor zugerechnet.
} 


\subsection{Finanzierung von Forschung und Entwicklung im Hochschulsektor}

Die Hochschul-F\&E in Österreich absorbiert einen international unübertroffen hohen Anteil der öffentlichen F\&E-Ausgaben und ist fast zur Gänze öffentlich finanziert. Entsprechend gering ist gemessen an den finanziellen Strömen die Vernetzung des Hochschulsektors mit dem Unternehmenssektor bzw. mit dem Ausland.

Einer landläufigen Auffassung zufolge wird an den Hochschulen Grundlagenforschung durchgeführt, die wiederum, weil ihre Ergebnisse den Charakter eines öffentlichen Gutes haben, öffentlich finanziert wird. Abbildung 1.3.1 zeigt folgendes:

- Das Ausmaß, in dem der öffentliche Sektor seine F\&E-Mittel auf den Hochschulsektor konzentriert, variiert sehr stark. Österreich nimmt mit Abstand hier die Spitzenposition ein. Der Hochschulsektor absorbiert in Österreich über $70 \%$ der gesamten öffentlichen Forschungsmittel, während es im internationalen Durchschnitt weniger als $50 \%$ sind.

- Die Hälfte aller Länder finanzieren über $90 \%$ der im Hochschulsektor ausgeführten F\&E durch die öffentliche Hand. Österreich führt mit $97 \%$ diese Gruppe jedoch an. Unmittelbare Folgerung aus dieser Tatsache ist die geringe Vernetzung der österreichischen Hochschulforschung mit anderen Sektoren, insbesondere mit dem Unternehmenssektor und dem Ausland.

Abbildung 1.2.4: Ausländische Finanzierung von Unternehmens-F\&E, 1993

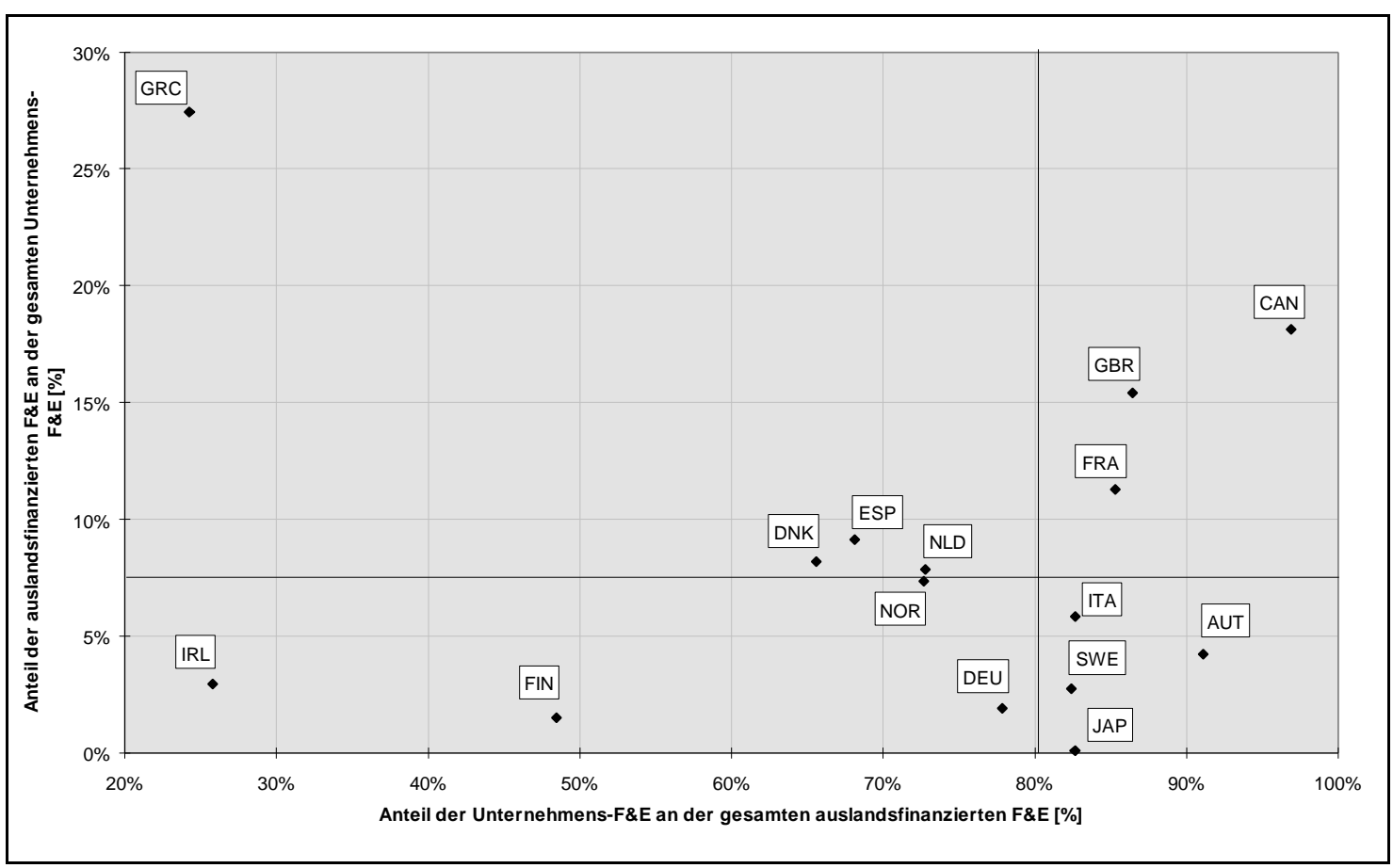

Q: OECD-MSTI, ÖSTAT. 
Abbildung 1.3.1: Öffentlich finanzierte F\&E im Hochschulsektor, 1993

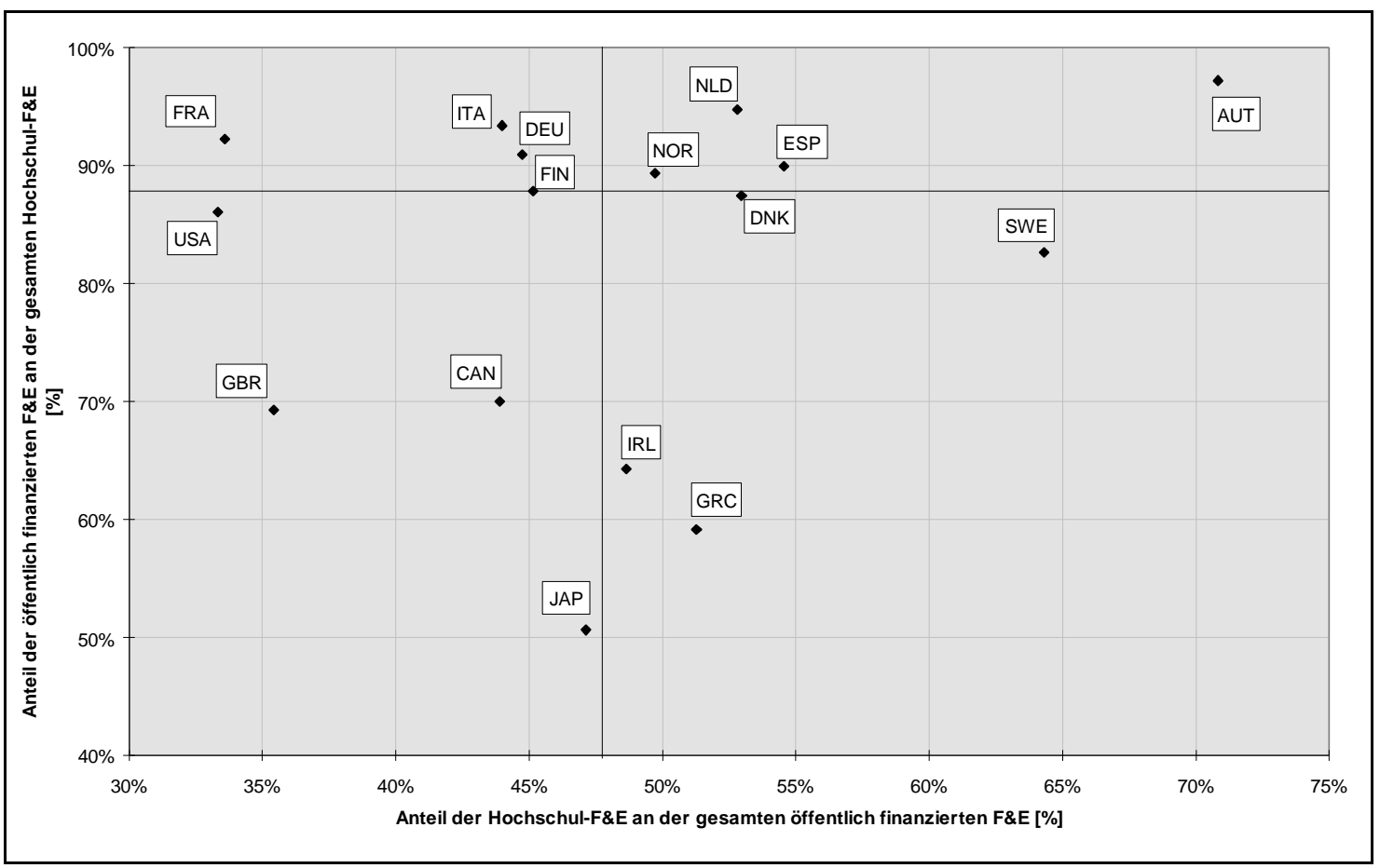

Q: OECD-MSTI, ÖSTAT.

Der Dominanz des öffentlichen Sektors bei der Finanzierung von Hochschul-F\&E entspricht ein geringes Engagement des Unternehmenssektors. Dies läßt sich aus Abbildung 1.3.2 ablesen.

- Die österreichischen Unternehmen lassen im Ausmaß von 1,4\% ihrer Forschungsausgaben F\&E an den österreichischen Hochschulen durchführen. Sie liegen damit deutlich unter dem Durchschnitt, jedoch gleichauf mit dem Unternehmenssektor in den USA und sogar vor jenem Frankreichs, der Niederlande, Dänemarks und Japans.

- Generell, d. h. über alle Länder hinweg, fällt der geringe Anteil von durchschnittlich 2,3\% der privaten F\&E-Mittel auf, der an die Hochschulen geht.

- Betrachtet man die Anteile der unternehmensfinanzierten F\&E an den Forschungsbudgets der Hochschulen, so liegt der Durchschnitt bei etwas über $5 \%$. Sieht man von Kanada ab (11\%), so sind diese nicht höher als $8 \%$ und nicht geringer als $2 \%$. An genau dieser unteren Schwelle liegt Österreich mit $2 \%$, was den bereits erhobenen Befund der geringen Vernetzung noch einmal in Erinnerung bringt.

- Es ist wenig riskant, aus diesen Zahlen, national wie international, den Schluß zu ziehen, daß Auftragsforschung im Sinne von unternehmensfinanzierter F\&E an den Hochschulen, von geringer Relevanz ist und Technologietransfer über andere Mechanismen als über Auftragsforschung vermittelt wird bzw. werden muß. 


\subsection{Unternehmensfinanzierte Forschung und Entwicklung im öffentlichen Sektor}

Während "F\&E im Unternehmenssektor" und "F\&E im Hochschulsektor" weitgehend selbsterklärend sind, ist die Kategorisierung von "F\&E im öffentlichen Sektor" im internationalen Vergleich nicht immer konsistent. In Österreich handelt es sich dabei um F\&E, die insbesondere in "Bundesinstitutionen (unter Ausklammerung der im Hochschulsektor zusammengefaßten), Landes-, Gemeinde-, Kammerinstitutionen sowie Einrichtungen von Sozialversicherungsträgern, Museen und Krankenanstalten" durchgeführt wird. In Übereinstimmung mit den OECD-Richtlinien werden daher das Österreichische Forschungszentrum Seibersdorf und Joanneum Research in der amtlichen F\&E-Statistik zum Unternehmenssektor gezählt. Im Gegensatz dazu werden etwa das finnische VTT und das niederländische TNO dem öffentlichen Sektor zugerechnet.

Der größte Teil der Länder hat einen öffentlichen Forschungssektor, der wenig mit dem Unternehmenssektor verknüpft ist.

Abbildung 1.3.2: Unternehmensfinanzierte F\&E im Hochschulsektor, 1993

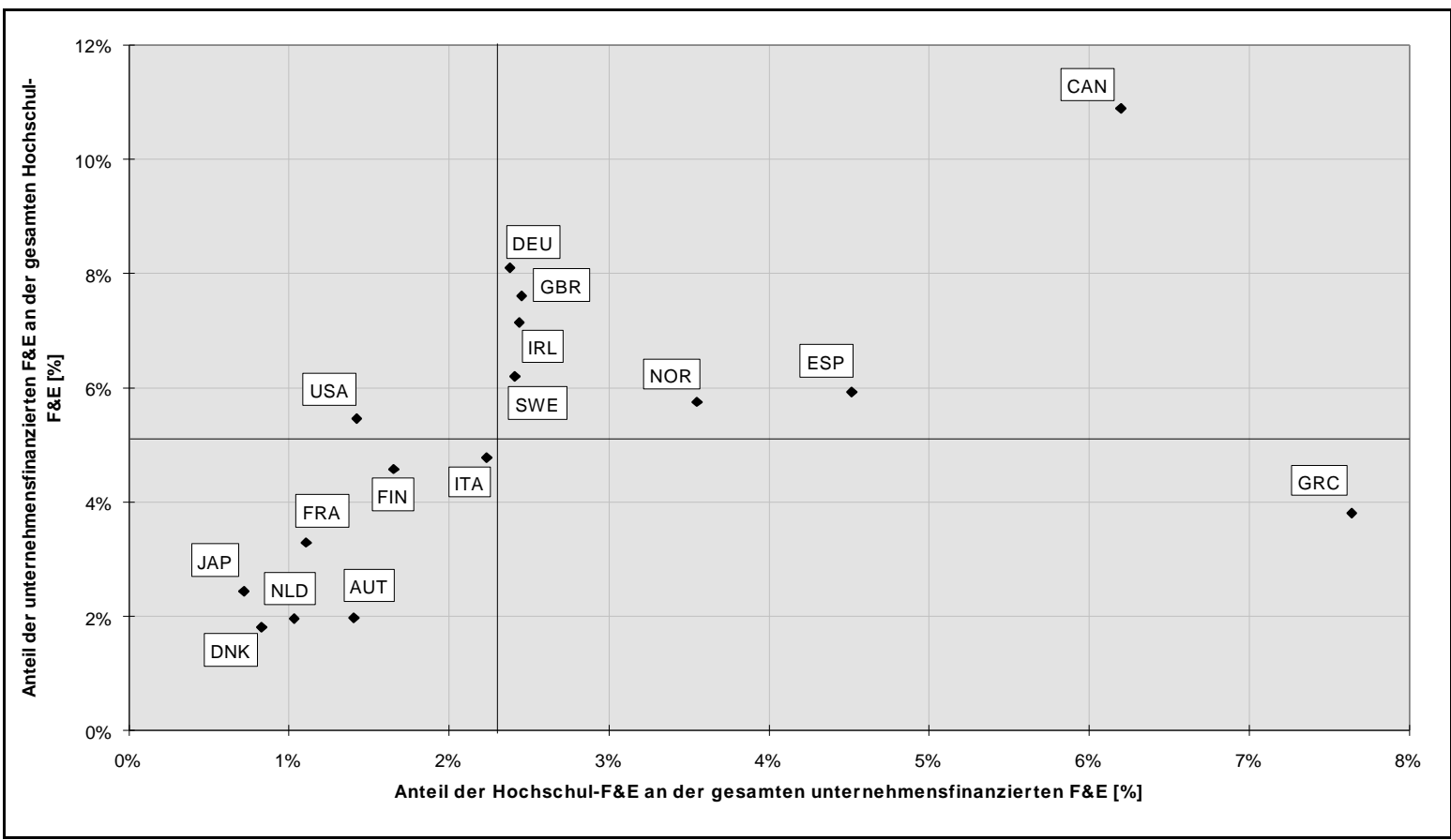

Q: OECD-MSTI, ÖSTAT. 
Abbildung 1.4.1: Unternehmensfinanzierte F\&E im öffentlichen Sektor, 1993

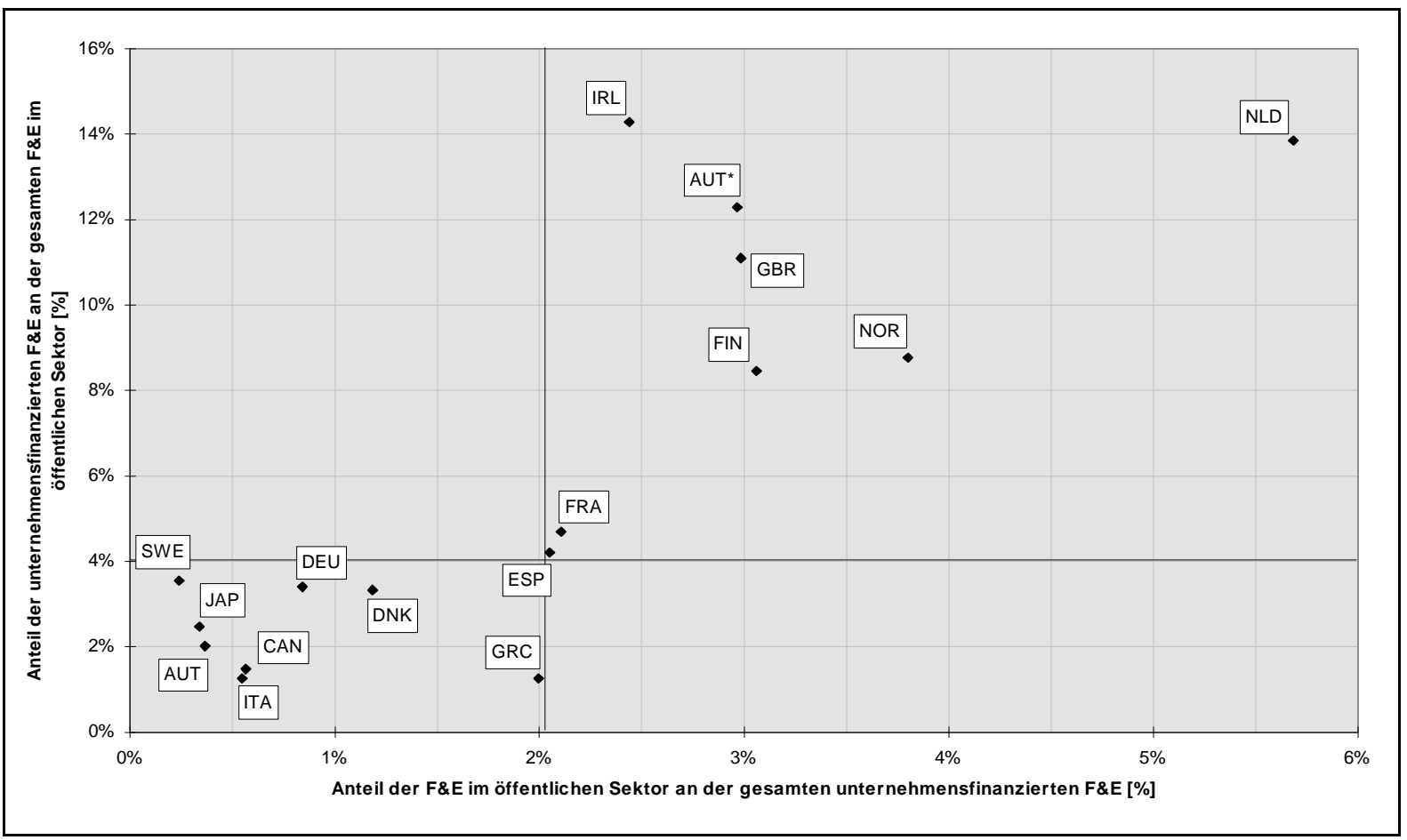

Q: OECD-MSTI, ÖSTAT.

Für die folgende Analyse sei nun neben der offiziellen Statistik (Position AUT) folgendes Gedankenexperiment (Position AUT*) durchgeführt: Das Forschungszentrum Seibersdorf und Joanneum Research werden dafür dem öffentlichen Sektor zugerechnet. Aus dem Vergleich der beiden Positionen AUT und AUT* in Abbildung 1.4.1 ergeben sich dann folgende Befunde:

- Basierend auf der Position AUT in Abbildung 1.4.1 ist die Forschung im öffentlichen Sektor in Österreich für den Unternehmenssektor weithin irrelevant, wie dies durch die geringen Zahlungsströme angezeigt wird. Lediglich 0,3\% der vom Unternehmenssektor finanzierten F\&E wird im öffentlichen Sektor ausgeführt. Dieser deckt damit $2 \%$ seines Budgets.

- Nach dem oben dargestellten Gedankenexperiment ändern sich diese Verhältnisse deutlich, wenn man das Forschungszentrum Seibersdorf und die Joanneum Research $\mathrm{GmbH}$ dem öffentlichen Sektor hinzurechnet. Dann kommt Österreich (Position AUT*) in jener Gegend zu liegen, in der Länder mit großen nationalen Forschungseinrichtungen wie Finnland, Großbritannien oder die Niederlande positioniert sind. 


\subsection{Die Vernetzung des Unternehmenssektors mit anderen Sektoren}

Österreich zählt zur Gruppe jener Länder mit eher geringer Interaktion des Unternehmenssektors mit anderen Sektoren. Der Anteil der außerhalb des Unternehmenssektors durchgeführten F\&E an der gesamten unternehmensfinanzierten F\&E ist in Österreich der geringste unter allen OECDLändern.

Eine besonders interessante Frage, die schon mehrfach berührt wurde, ist die jene nach dem Grad der Vernetzung des Unternehmenssektors mit anderen Sektoren. In Abbildung 1.5.1 wird dargestellt, welche Anteile der unternehmensfinanzierten F\&E in anderen Sektoren durchgeführt werden, also im Hochschulsektor, im öffentlichen Sektor oder im Ausland (horizontale Achse). Diesen wird gegenübergestellt, welche Anteile der vom Unternehmenssektor ausgeführten F\&E nicht von diesem finanziert wird, sondern insbesondere vom Staat bzw. vom Ausland. Dabei ergeben sich folgende Befunde:

- Es lassen sich zwei Gruppen von Ländern identifizieren: Solche mit einem hohen Grad an Interaktion, d. h. mit umfangreicheren Zahlungsströmen aus dem Unternehmenssektor an andere Sektoren und von anderen Sektoren an den Unternehmenssektor (Griechenland, Großbritannien, Kanada, Norwegen, Niederlande und Spanien) und solche mit geringer Interaktion (Italien, Dänemark, Deutschland, Schweden, Japan und Österreich). Es gibt eine klare Ausnahme (Frankreich) und einige Grenzfälle. Es läßt sich generell ein gewisser positiver Zusammenhang zwischen eingehender und ausgehender Finanzierung von F\&E feststellen. Dies bedeutet, daß in Ländern, in denen Unternehmens-F\&E in höherem Ausmaß öffentlich oder durch das Ausland finanziert wird, der Unternehmenssektor seinerseits auch vermehrt die Durchführung von F\&E in anderen Sektoren finanziert.

- Österreich zählt zu jener Gruppe, in der der Grad der Interaktion und Vernetzung zwischen dem Unternehmenssektor und anderen Sektoren eher gering ist. Hinsichtlich der externen Finanzierung von Unternehmens-F\&E liegt Österreich mit rund 14\% nahe am Durchschnitt von rund 17\%. Dagegen ist die Bereitschaft des Unternehmenssektors, F\&E anderswo durchführen zu lassen, extrem gering $(2 \%$ gegenüber 4,8\% im internationalen Durchschnitt) - folgt man der amtlichen Statistik (Position AUT). Berücksichtigt man hingegen oben entwickeltes Gedankenexperiment (Position AUT ${ }^{*}$ ), so erhöht sich der Wert von $2 \%$ auf $4,5 \%$. In dieser Sichtweise verzeichnet der österreichische Unternehmenssektor also eine durchschnittliche Vernetzung mit den übrigen Sektoren. Was das Verhältnis von eingehenden zu ausgehenden Finanzierungsströmen betrifft, so liegt ein (fiktives) Verhältnis von $3: 1$ vor - durchaus im Durchschnitt. 
Abbildung 1.5.1: Externe F\&E-Finanzierung des Unternehmenssektors und F\&EDurchführung für den Unternehmenssektor, 1993

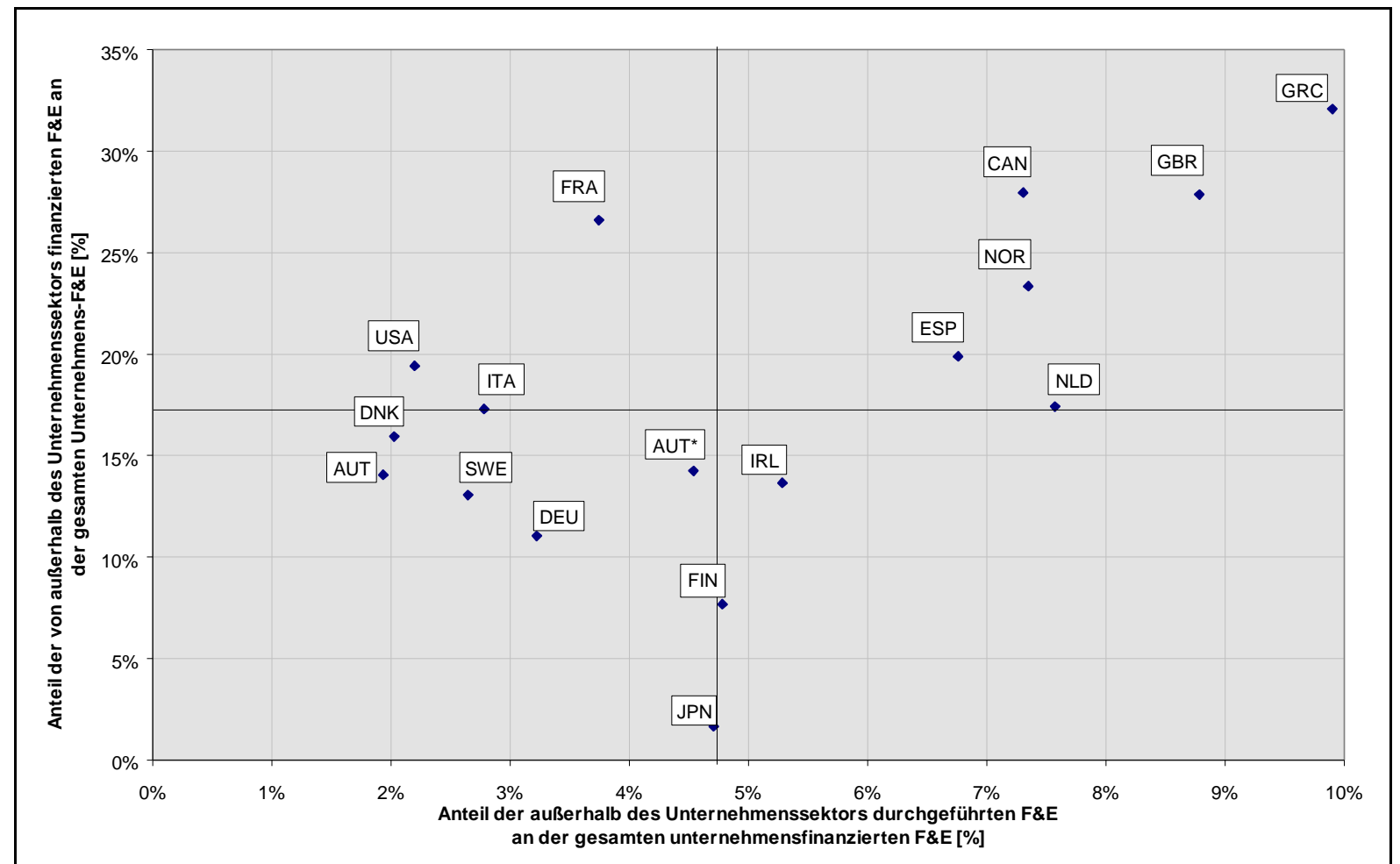

Q: OECD-MSTI, ÖSTAT. 


\section{Box 1: Österreichs Innovationssystem und Standortqualität}

Eine Befragung des WIFO unter Geschäftsführern und Vorständen der wichtigsten in Österreich tätigen Industrie- und einiger Dienstleistungsunternehmen bietet Einblicke in die Stärken und Schwächen des Industriestandorts Österreichs (Aiginger - Peneder, 1997). Unter den 15 bestbewerteten Faktoren der Standortqualität (Top-15) finden sich allerdings nur wenige, die sich auf Stärken des österreichischen Innovationssystems zurückführen lassen. Positiv bewertet wurden in diesem Zusammenhang die Verfügbarkeit hochqualifizierter bzw. gut ausgebildeter Arbeitskräfte und die Initiative und Innovationsbereitschaft der Führungskräfte. Die Top-5 unter den Faktoren der Standortqualität hingegen sind die politische Stabilität, die öffentliche Sicherheit, das Kulturund Freizeitangebot, die Umweltqualität und die Rechtssicherheit. Schwächen im Innovationssystem werden als Defizite der Standortqualität wahrgenommen. So etwa werden unter den 15 Standortfaktoren mit der schlechtesten Bewertung (Low-15) Mängel hinsichtlich Telekommunikationskosten, Verfügbarkeit von Risikokapital, Anpassungsfähigkeit und Reformbereitschaft, Forschungseinrichtungen von internationalem Format, Akzeptanz neuer Technologien und Öffnung der Telekommunikationsmärkte genannt.

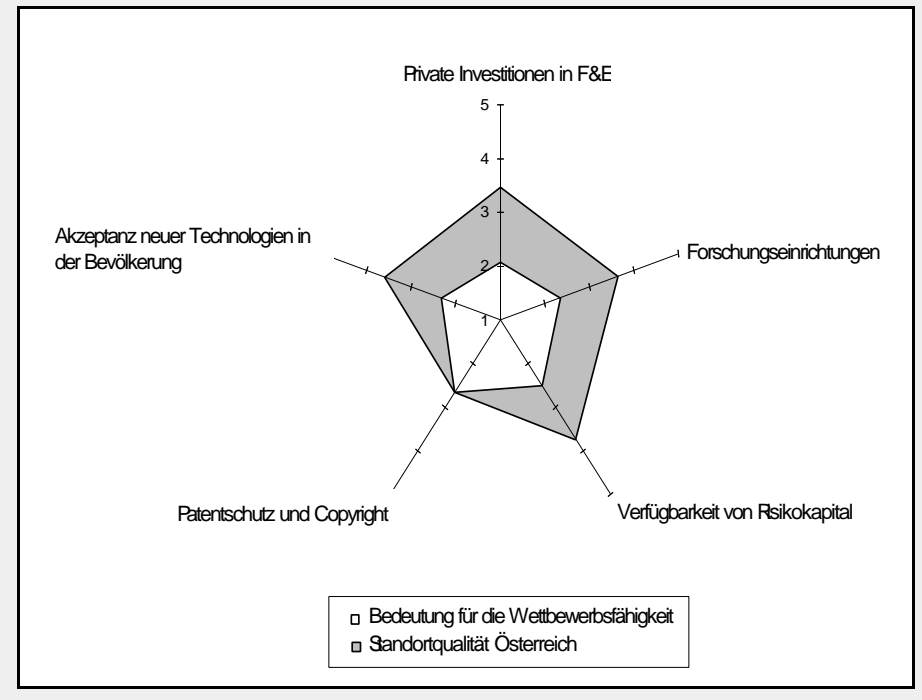

Wie die Abbildung zeigt, besteht eine Diskrepanz zwischen der Bedeutung, die die befragten Manager technologierelevanten Faktoren für die Wettbewerbsfähigkeit eines Landes wie Österreich zuordnen ("Bedeutung für die Wettbewerbsfähigkeit"), und der Bewertung der wahrgenommenen faktischen "Standortqualität Österreichs". Die Bewertung erfolgt anhand einer "Notenskala" von 1 ("hoch") bis 5 ("niedrig"). Die privaten F\&EInvestitionen, die Forschungseinrichtungen, die Verfügbarkeit von Risikokapital und die Akzeptanz neuer Technologien entsprechen demnach nicht ihrer Bedeutung für die 
Wettbewerbsfähigkeit. Lediglich im Bereich Patentschutz und Urheberrecht entspricht die derzeitige Lage den Anforderungen. 


\section{Spezialisierungsmuster des österreichischen Innovationssystems}

\section{$2.1 \quad$ Humanressourcen}

Die Qualifikation der Arbeitskräfte ist ein zentraler Faktor für die internationale Konkurrenzfähigkeit der Unternehmen und für die langfristigen Wachstumschancen einer Volkswirtschaft. Die Qualifikation der Arbeitskräfte ist ihrerseits wiederum eng verknüpft mit der Struktur und Qualität des Ausbildungssystems. Dabei ist jedoch zu berücksichtigen, daß Entwicklungen im Bildungssystem nicht unvermittelt auf das Beschäftigungssystem durchschlagen. Eine einseitige Fixierung auf die Ausbildungs- bzw. Angebotsseite Stichwort Akademikerquote - ist unter technologie- oder beschäftigungspolitischen Gesichtspunkten nicht zielführend.

Die Verfügbarkeit hochqualifizierter und gut ausgebildeter Arbeitskräfte wird allgemein zu den "Standortvorteilen" Österreichs gezählt ${ }^{4}$ ). Andererseits gibt es jedoch sowohl begründete Zweifel an der Effizienz als auch Fragen bezüglich der Struktur des Qualifikationssystems.

Hochqualifizierte und gut ausgebildete Arbeitskräfte zählen zu den wesentlichen Standortvorteilen Österreichs.

Nach einem internationalen Vergleich der OECD liegt Österreich gemessen am Anteil der öffentlichen und privaten Ausgaben für Bildungseinrichtungen am BIP mit 5,6\% (1994) unter 19 OECD-Ländern an 13. Stelle (siehe Abbildung 2.1.1).

Hinter den globalen Daten verbergen sich jedoch durchaus unterschiedliche Spezialisierungen der nationalen Qualifikationssysteme. So etwa unterscheidet sich das Qualifikationssystem Österreichs von jenem anderer Länder dadurch, daß der Anteil der Sekundarschulbildung (allgemeinbildende höhere Schulen, berufsbildende mittlere und höhere Schulen etc.) überdurchschnittlich und jener der Tertiärschulbildung (Hochschulen, Akademien etc.) unterdurchschnittlich ist. Bemerkenswert ist weiters der im internationalen Vergleich hohe Anteil der Berufsbildung in Relation zur Allgemeinbildung innerhalb der oberen Sekundarstufe (darunter Oberstufe der AHS, berufsbildende mittlere und höhere Schulen). Zur Zusammensetzung der Bevölkerung zwischen 25 und 64 Jahren nach dem höchsten abgeschlossenen Ausbildungsniveau siehe Abbildung 2.1.2.

Die Struktur der Absolventen gibt einen Hinweis auf das Angebot von Arbeitskräften der entsprechenden Qualifikationen. Bemerkenswert ist in diesem Zusammenhang, daß Österreich unter 20 OECD-Ländern - vor den Niederlanden - den zweitgeringsten Anteil

$\left.{ }^{4}\right)$ Siehe etwa die in Box 1 dargestellten Ergebnisse der Manager-Befragung von Aiginger-Peneder (1997). 
natur- und ingenieurwissenschaftlicher Neu-Absolventen der Universitäten an den Erwerbstätigen zwischen 25 und 34 Jahren aufweist (siehe Abbildung 2.1.3).

Österreich weist unter 20 OECD-Ländern den zweitniedrigsten Anteil natur- und ingenieurswissenschaftlicher Universitätsabsolventen an den Erwerbstätigen zwischen 25 und 34 Jahren auf.

Eine neuere Untersuchung über die Entwicklung in den siebziger und achtziger Jahren kam zu dem überraschenden Ergebnis, daß das leicht steigende Angebot an Ingenieuren mit Universitätsausbildung in sinkendem Maße in Unternehmen des Produktionssektors beschäftigt wird ${ }^{5}$. Die Autoren der Studie sehen darin einen Hinweis auf Verzerrungen im Verhältnis zwischen den Universitäten und dem Unternehmenssektor.

Die Einrichtung eines Fachhochschulsystems bringt eine sinnvolle Erweiterung des Qualifikationsangebots, insbesondere in wirtschaftsrelevanten Bereichen. Darüber hinaus könnte sich die neue Konkurrenz durch die Fachhochschulen auf Sicht positiv auf die Leistungsfähigkeit der Universitäten auswirken.

Abbildung 2.1.1: Bildungsausgaben (öffentlich und privat) in Prozent des BIP, 1994

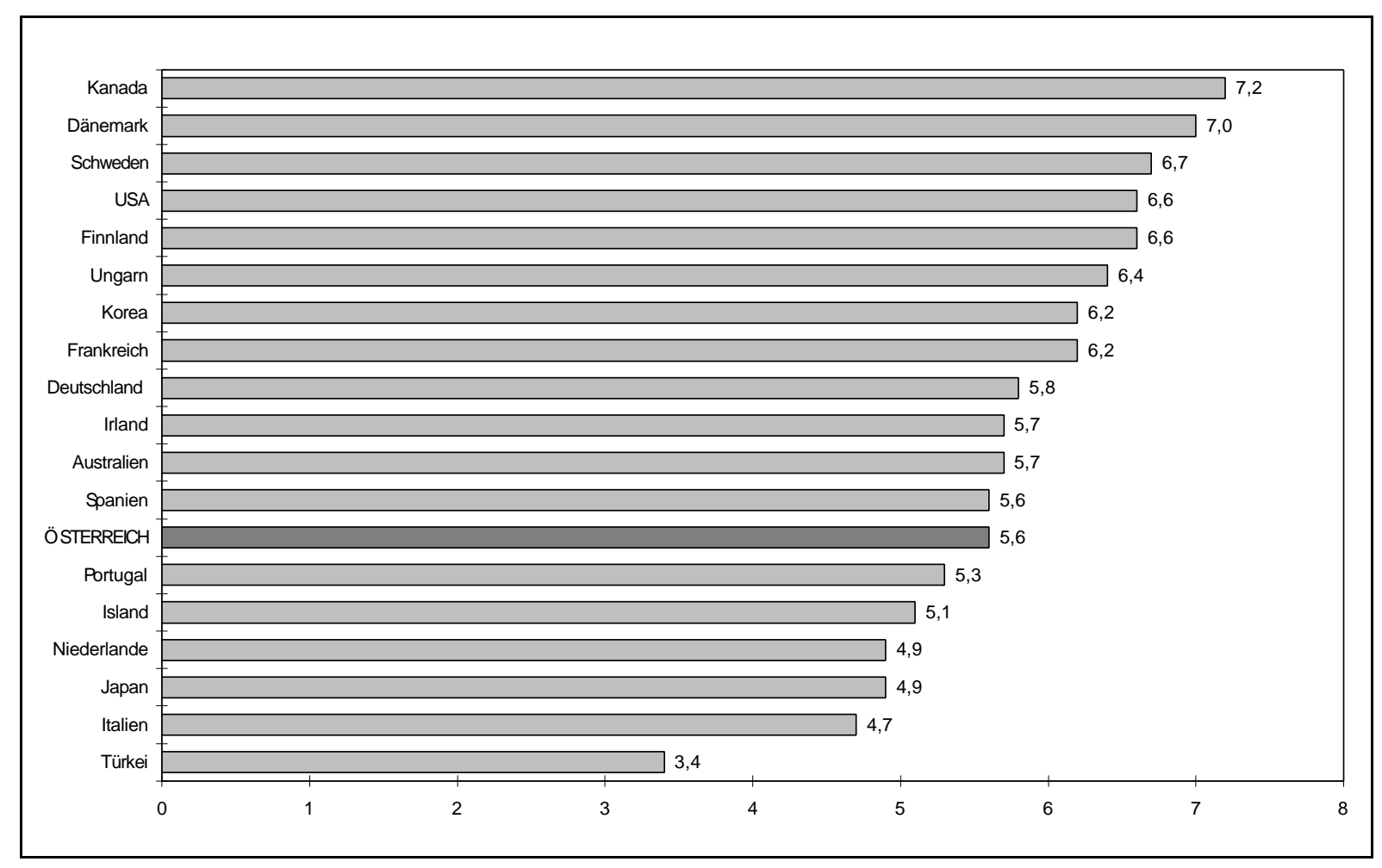

Q: OECD (1997).

$\left.{ }^{5}\right)$ Lassnigg - Pollan (1996). 
Abbildung 2.1.2: Ausbildungsstruktur der 25- bis 64jährigen Bevölkerung, 1995

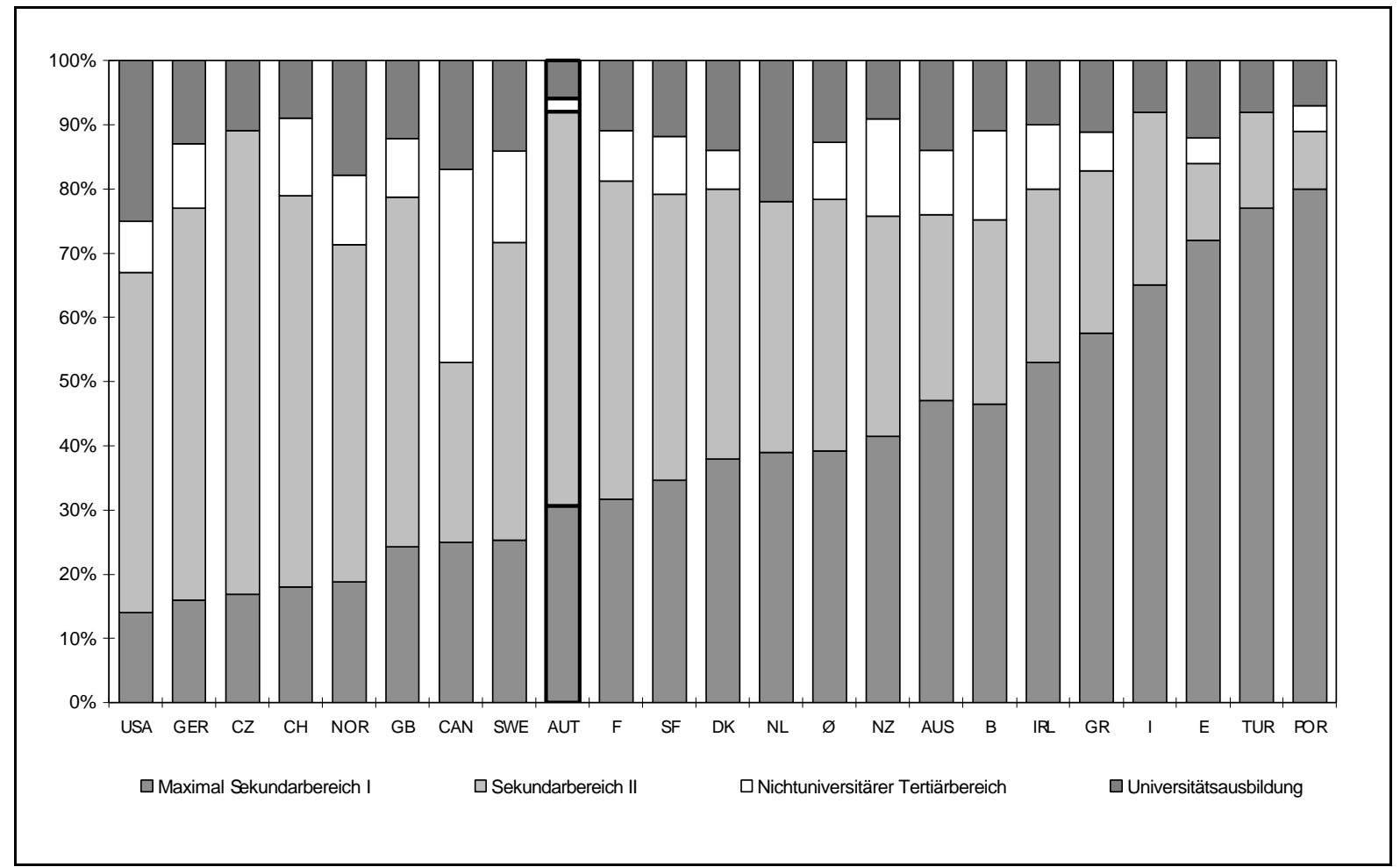

Q: OECD (1997).

\subsection{Patentspezialisierung}

Der Schutz von technischen Erfindungen durch Patente schafft Marktzugangsbarrieren und damit ein zeitliches und räumliches Verfügbarkeitsmonopol. Gleichzeitig ist mit der Anmeldung eines Patentes eine inhaltliche Offenlegung verbunden, wodurch Informationen über die Erfindung allgemein zugänglich gemacht werden. Patentstatistiken zählen neben F\&E-Statistiken zu den am häufigsten verwendeten empirischen Grundlagen der Innovationsforschung. F\&E-Daten sind Inputindikatoren, d. h. sie messen den Umfang von Inputs, die im Innovationsprozeß eingesetzt werden. Patentdaten hingegen werden oft als "Throughput"-Indikatoren bezeichnet, da sie den Übergang von der Erfindung zur Innovation markieren. Outputindikatoren schließlich sind etwa Kennzahlen über neue Produkte und Prozesse. Trotz mancher Probleme bei der Interpretation von Patentstatistiken ist ihre Funktion als Frühindikator für Innovationen unbestritten.

In der evolutionären Innovationstheorie werden Patente als ein Indikator für technologische Lernprozesse betrachtet, die zu einem wesentlichen Teil aus der Gewinnung nicht kodifizierbaren Wissens ("tacit knowledge") bestehen, das im Zuge der Entwicklung eines Patentes generiert wird. Auf der Makro-Ebene eignen sich Patentstatistiken dazu, verschiedene Länder ("Nationale Innovationssysteme") hinsichtlich ihrer technologischen 
Spezialisierung zu vergleichen und ihre Position im internationalen Technologiewettlauf festzustellen.

Der Anteil österreichischer Patenterteilungen am Europäischen Patentamt bewegt sich im Zeitraum 1987-1995 um einen Wert von 1,2\%.

Die Etablierung des Europäischen Patentamtes als überregional anerkannter Institution ermöglicht internationale Vergleiche der Patentstrukturen bzw. -spezialisierungen auf einheitlicher Grundlage. Der Anteil österreichischer Patenterteilungen schwankte in den letzten Jahren um einen Wert von rund 1,2\%. Im Jahr 1995 wurden 430 österreichische Patente am Europäischen Patentamt erteilt. Damit lagen die Erteilungen deutlich unter jenen des Vorjahres (siehe Abbildung 2.2.1).

Die Patentintensität (Anzahl der Patentanmeldungen pro Kopf) erreicht in der Schweiz und den skandinavischen Ländern die höchsten Werte. Österreich befindet sich im unteren Mittelfeld der europäischen Länder.

Abbildung 2.1.3: Natur- und ingenieurwissenschaftliche Neu-Absolventen der Universitäten je 100.000 Erwerbstätige im Alter von 25 bis 34 Jahre, 1995

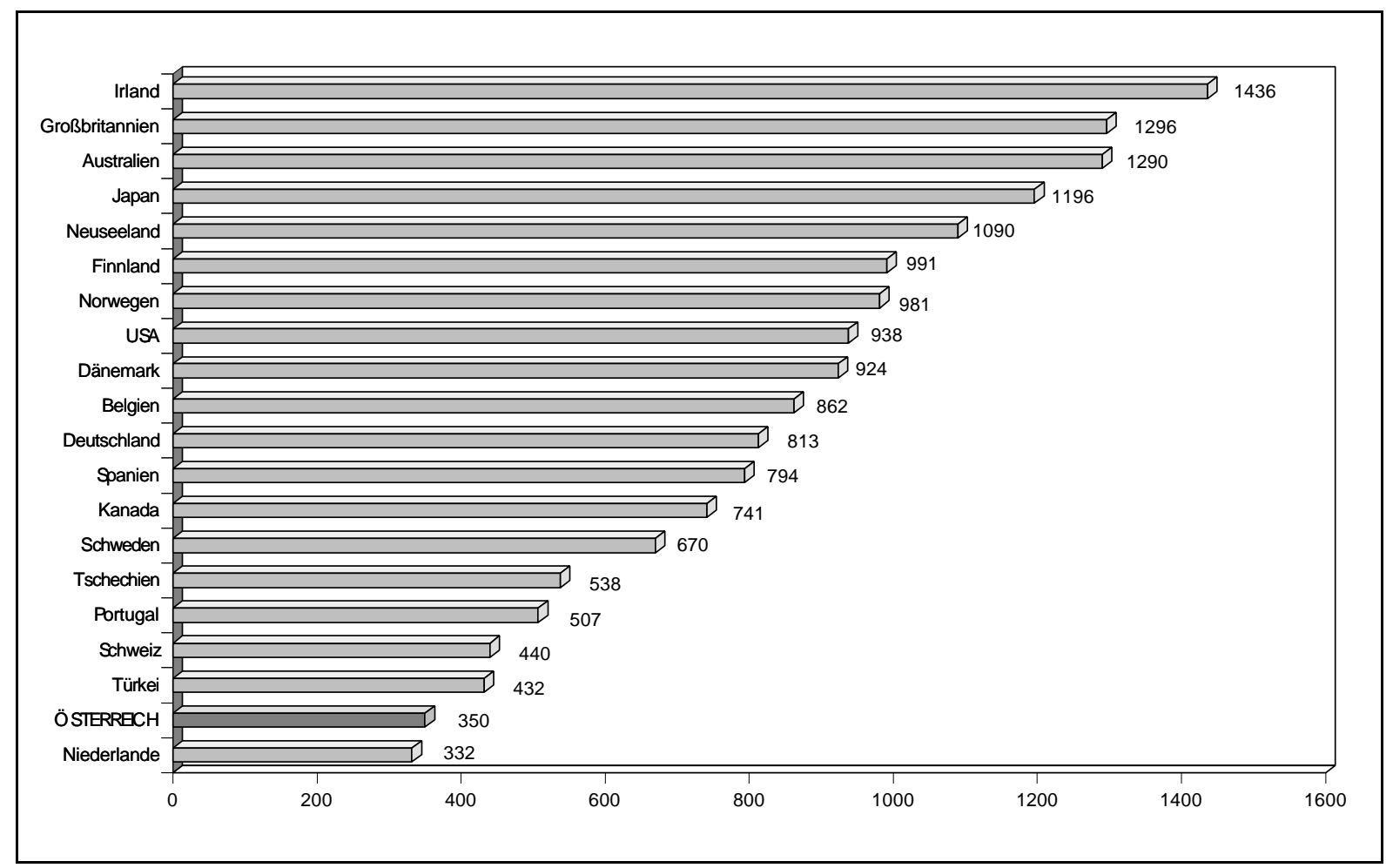

Q: OECD (1997). 
Abbildung 2.2.1: Österreichische Patente am Europäischen Patentamt, 1987-1995

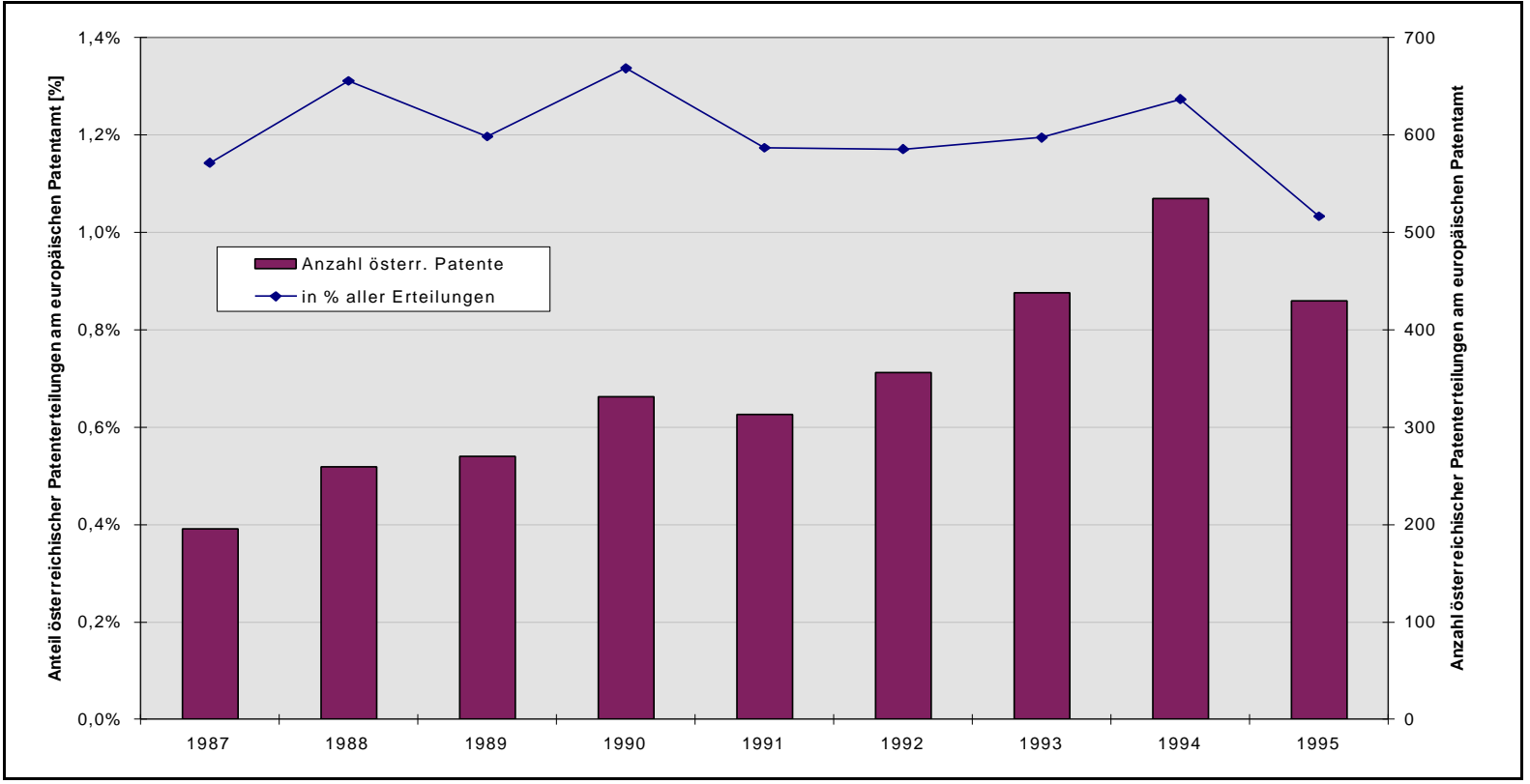

Q: Europäisches Patentamt (1987-1995).

Abbildung 2.2.2: Anzahl inländischer und externer Patentanmeldungen bezogen auf das BIP bzw. auf 100.000 Einwohner, 1995

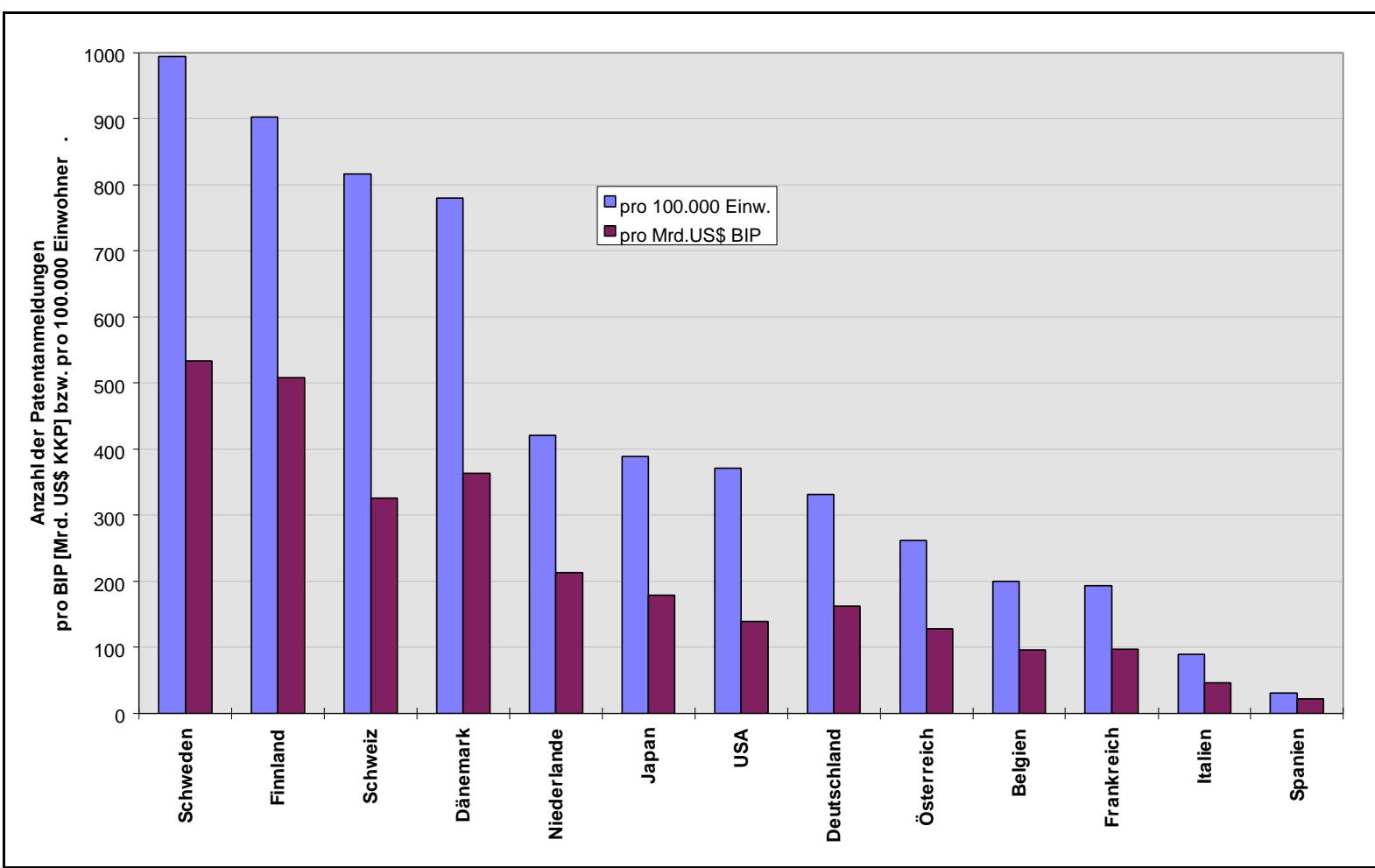

Q: OECD Main Main Science and Technology Indicators, eigene Berechnungen. 
Abbildung 2.2.2 gibt die Summe aus inländischen (von Inländern am jeweiligen nationalen Patentamt getätigten) und externen (von Inländern im Ausland getätigten) Patentanmeldungen wieder. Da ein Patent zumeist in mehreren Ländern angemeldet wird, sind Mehrfachzählungen möglich. Dies wird hier bewußt in Kauf genommen, da durch Patente unter anderem Exportmärkte geschützt werden. Kleine Länder mit für den Weltmarkt unbedeutenden nationalen Patentämtern werden dadurch jedoch überbewertet, da für kleine Volkswirtschaften internationale Beziehungen wichtiger sind als für Länder mit einem großen nationalen Markt.

Nach Angaben der OECD wies im Jahr 1995 Schweden mit nahezu 1.000 Patentanmeldungen je 100.000 Einwohner die höchste Patentintensität auf, gefolgt von Finnland, Schweiz, Dänemark und den Niederlanden. Österreich befand sich mit 262 Patentanmeldungen im unteren Mittelfeld der europäischen Länder, aber noch vor Ländern wie Belgien, Frankreich und Italien.

Das Verhältnis von externen zu inländischen Patentanmeldungen eines Landes liefert einen Hinweis auf die potentielle internationale Diffusion seiner Innovationen und kann somit als Fähigkeit seines Innovationssystems zur technologischen Internationalisierung interpretiert werden.

Die Patentanmeldungen weisen auf eine starke Tendenz zur Internationalisierung hin. Am weitesten ist diese in kleinen offenen Volkswirtschaften wie in den Niederlanden, Belgien, den skandinavischen Ländern und in der Schweiz fortgeschritten. Österreichs Internationalisierungsgrad ist im Vergleich dazu noch eher gering, nimmt aber rasch zu.

Im Lauf der achtziger Jahre kam es in allen betrachteten Ländern zu einer bemerkenswerten Internationalisierung der Patentanmeldungen, die sich in den neunziger Jahren noch beschleunigt hat. In besonderem Maße gilt das für kleine offene Volkswirtschaften wie Belgien, die Niederlande, die skandinavischen Länder sowie die Schweiz. In den Niederlanden, dem Land mit traditionell starker internationaler Ausrichtung, stieg das Verhältnis externe/inländische Anmeldungen von 6,1 im Jahr 1981 auf 34,3 im Jahr 1995. In Österreich stieg dieses Verhältnis im gleichen Zeitraum von 2,0\% auf 9,6\%. Weniger stark internationalisiert haben sich die Patentanmeldungen in einer Gruppe von Ländern mit großem Binnenmarkt wie Deutschland, Frankreich, USA, aber auch Spanien. Bemerkenswert ist der nach wie vor geringe Internationalisierungsgrad Österreichs, der eher mit der zweiten Gruppe konform geht und nur halb so hoch ist wie in der vergleichbaren Gruppe der kleinen offenen Volkswirtschaften (siehe Abbildung 2.2.3).

Die relative Position der Länder im internationalen technologischen Wettbewerb kann qualitativ an Technologieprofilen wie in Abbildung 2.2.4 abgelesen werden. Darin sind die Patentanmeldungen nach Herkunftsland erfaßt und über den Technologiefeldern ${ }^{6}$ ) als

${ }^{6}$ ) Definition der Technologiefelder gemäß Europäischem Patentamt. 
Prozentanteil an der jeweiligen Gesamtzahl aufgetragen. Die österreichische Patentstruktur wird somit im Vergleich zur Verteilung der Patentanmeldungen aller Ländern deutlich.

Die österreichischen Patentanmeldungen sind überaus stark auf das Technologiefeld "Bauwesen" konzentriert. Hochtechnologiebereiche wie Instrumente, Elektrotechnik, Elektronik und Nachrichtentechnik spielen im Vergleich zur Mehrheit der untersuchten Länder eine relativ geringe Rolle.

Die internationale Patentstruktur ist jene eines Aggregats aus zwölf Ländern: Belgien, Deutschland, Frankreich, Großbritannien, Italien, Japan, die Niederlande, Österreich, Schweden, Schweiz, Spanien und USA. In vier dieser Länder (Belgien, Großbritannien, Japan, USA) ist das Technologiefeld "Instrumente I" das bedeutendste; da sich darunter auch die in absoluten Zahlen größten Patentanmelder befinden, ist "Instrumente I" mit annähernd $10 \%$ der gesamten Anmeldungen auch das wichtigste Technologiefeld für die Gesamtheit dieser Länder. Es folgen die Technologiefelder "Elektrotechnik", das in drei Ländern (Deutschland, Frankreich, Niederlande) an der Spitze liegt, und "Organische Chemie", auf das zwei Länder (Schweiz, Spanien) spezialisiert sind.

Abbildung 2.2.3: Verhältnis externer zu inländischen Patentanmeldungen in den Jahren 1979, 1984, 1989, 1992 und 1995 im internationalen Vergleich

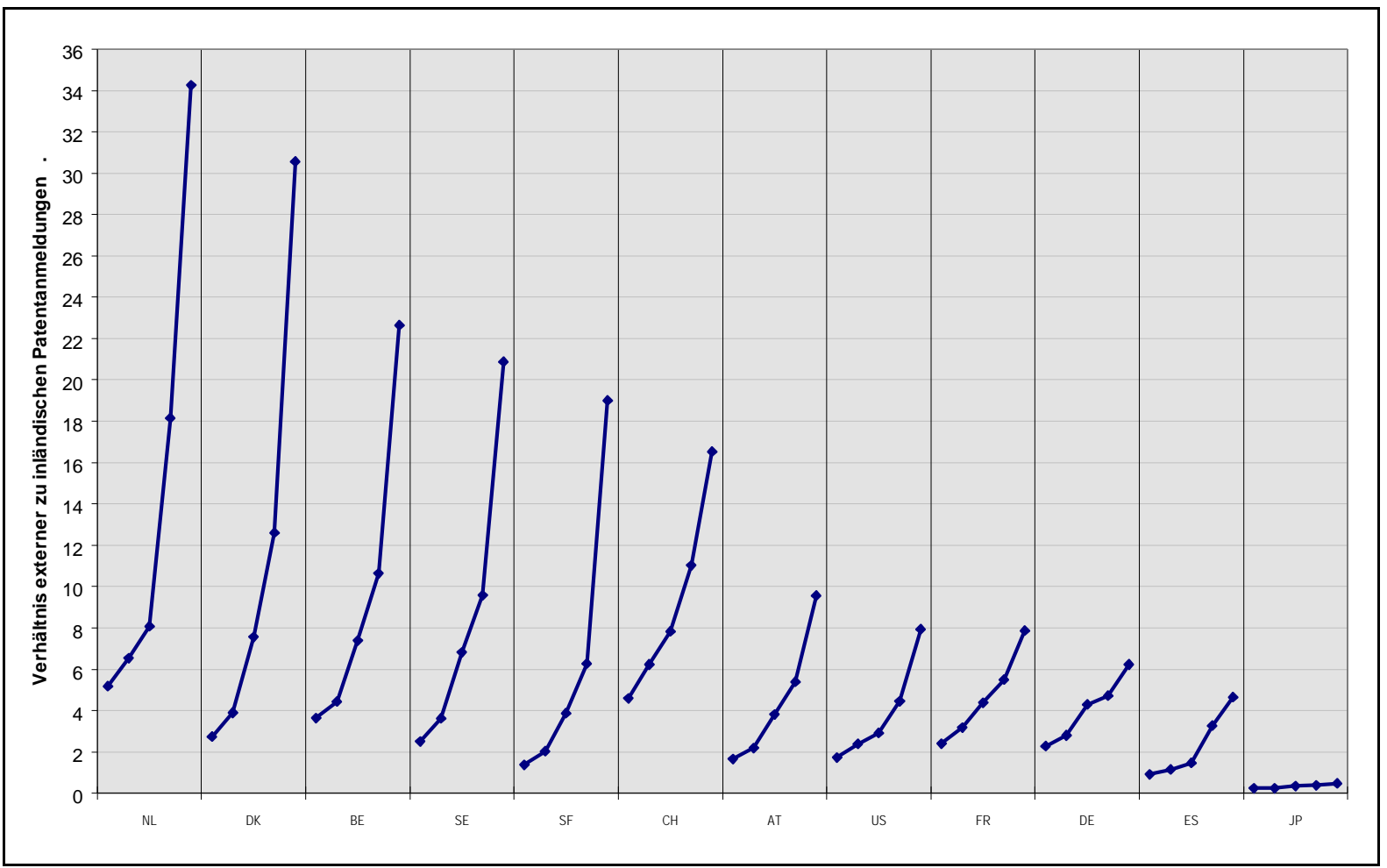

Q: OECD Main Science and Technology Indicators, eigene Berechnungen. 
Abbildung 2.2.4: Patentanmeldungen am Europäischen Patentamt nach Technologiefeldern in Prozent der jeweiligen Gesamtsumme, 1987 bis 1994

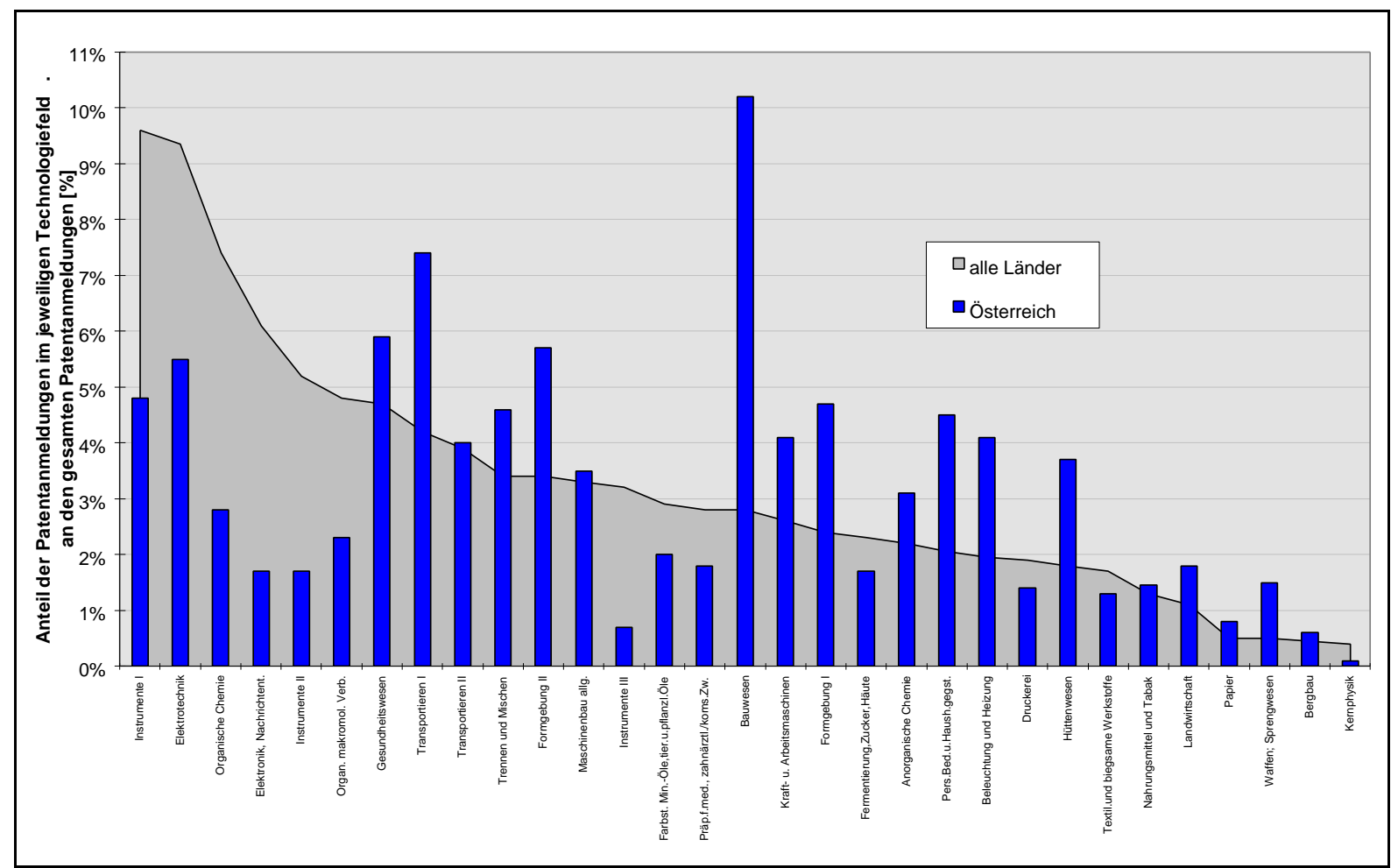

Q: Gassler et al. (1996).

Bemerkenswert ist die Tatsache, daß in Österreich mit dem Technologiefeld "Bauwesen" ein Feld an der Spitze steht, das in keinem anderen Land unter den vier wichtigsten Technologiefeldern zu finden ist. Dieses Feld ist sehr breit definiert und umfaßt alle Baubereiche bis hin zu Schlössern, Beschlägen und Leitern. In manchen dieser Bereiche hat Österreich patentintensive Unternehmen, die z. T. in Marktnischen Weltgeltung aufweisen. Trotzdem deutet die Tatsache, daß in Österreich Hochtechnologiebereiche wie Instrumente, Elektrotechnik, Elektronik und Nachrichtentechnik im Gegensatz zur Mehrheit der betrachteten Länder keine besondere Rolle spielen, auf erhebliche technologische Schwächen hin.

Die evolutionäre Theorie der Innovation betont den kumulativen und pfadabhängigen Charakter der technologischen Entwicklung. Gemeinsam mit externen Effekten und steigenden Skalenerträgen führen technologische Lerneffekte dazu, daß einzelne Länder (bzw. Unternehmen) einmal eingeschlagene technologische Pfade nicht ohne weiteres verlassen (können).

Die Patentdaten weisen auf eine ausgeprägte Stabilität nationaler Spezialisierungsmuster hin. Dies konnte auch anhand der österreichischen Patente beim Europäischen Patentamt gezeigt werden. 
In einer Reihe von empirischen Analysen wird diese Hypothese durch den Nachweis einer ausgeprägten zeitlichen Stabilität der nationalen Spezialisierungsmuster gestützt. Auch für Österreich wurde eine ausgeprägte zeitliche Persistenz des technologischen Spezialisierungsmusters nachgewiesen ${ }^{7}$ ). Auf diese Weise konnte die These von der Pfadabhängigkeit technologischen Wandels auch am Beispiel des österreichischen Innovationssystems bestätigt werden.

Die Unterschiede in den Marktbedingungen (Marktkonzentration, Nachfragewachstum etc.) sowie den technologischen Möglichkeiten führen zu spezifischen, branchentypischen Mustern des Innovationsverhaltens. So sind die Patentaktivitäten in Österreich stark auf einige wenige Branchen und Technologiefelder konzentriert, ein Umstand, der im übrigen auch in F\&E-Statistiken und bei Innovationszählungen zutage tritt.

Die Spezialisierung Österreichs auf das Bauwesen geht auch aus Daten des österreichischen Patentamtes hervor. Die Elektro- sowie die Maschinen- und Stahlbauindustrie sind die patentintensivsten Branchen in Österreich.

Die Patentaktivitäten der heimischen Industrie (Unternehmen mit 20 und mehr Beschäftigten) von 1987 bis 1994 wurden anhand von Daten des österreichischen Patentamtes untersucht und nach Branchen und Technologiefeldern analysiert ${ }^{8}$ ). Danach entfallen etwa 67\% der gesamten Patente (6.962 Anmeldungen) auf die vier patentstärksten Branchen Maschinen- und Stahlbauindustrie, Elektroindustrie, Eisen- und Metallwarenindustrie, sowie chemische Industrie. Der Anteil der patentierenden Unternehmen an der Gesamtheit der Unternehmen einer Branche reicht von 2,7\% in der Bekleidungsindustrie bis zu einem Maximum von 57,1\% in der eisenerzeugenden Industrie.

Hinsichtlich der Patentintensität (z. B. Anzahl der Patente pro 1.000 Beschäftigten oder Anzahl der Patente pro Unternehmen) zeigen sich ebenfalls beträchtliche Unterschiede zwischen den Branchen. Bezogen auf 1.000 Beschäftigte kommen im österreichischen Durchschnitt etwa 10 Patente. Unter den Branchen weist die Bekleidungsindustrie mit 0,5 Patenten die geringste Intensität auf, Spitzenreiter ist die Maschinen- und Stahlbauindustrie mit 23,8 Patenten je 1.000 Beschäftigte. Bezogen auf Unternehmen ergibt sich ein ähnlich heterogenes Bild: Durchschnittlich wurden im Beobachtungszeitraum 1,3 Patente pro Unternehmen angemeldet. An der Spitze liegt mit 5,4 Patenten die Elektroindustrie, gefolgt von der eisenerzeugenden Industrie mit 5,1 und der Maschinenund Stahlbauindustrie mit 3,5 Patenten pro Unternehmen.

Übersicht 2.2.5 zeigt eine Branchen-Technologiefelder-Matrix, in welcher die Zahl der Patente angegeben ist, die von einer Branche in einem bestimmten Technologiefeld angemeldet wurden. Daraus ist ersichtlich, welche Branche in welchem Ausmaß zur

\footnotetext{
$\left.{ }^{7}\right)$ Gassler et al. (1996). Die Autokorrelation der Technologieprofile Österreichs im Zeitraum 1987-1994 (Datenbasis: Europäisches Patentamt) ist ausgesprochen hoch.

$\left.{ }^{8}\right)$ Gassler et al. (1996).
} 
Generierung von Innovationen beiträgt. Darüber hinaus läßt sich die technologische Spezialisierung einer Branche feststellen bzw. umgekehrt untersuchen, welche Branchen zum Fortschritt in einem bestimmten Technologiefeld beitragen.

Die Aufschlüsselung der Patentierungen nach Technologiefeldern ergibt ebenfalls eine starke Konzentration. Die größte Zahl an Patenten findet sich mit 712 Anmeldungen im Technologiefeld "Bauwesen", einem äußerst heterogenen Feld, dem viele Bereiche der Infrastruktur (Wasserversorgung, Kanalisation, Straßen- und Eisenbahnbau etc.) bis hin zu Bereichen wie Schlösser und Beschläge zugeordnet sind und das nicht mit der Bauwirtschaft allein in Verbindung gebracht werden kann. Tatsächlich wird das Technologiefeld "Bauwesen" am stärksten von der Eisen- und Metallwarenindustrie bestimmt, gefolgt von der Maschinen- und Stahlbauindustrie und der Steine- und Keramikindustrie. Diese drei Branchen sind für zwei Drittel der Patentierungen in diesem Technologiefeld verantwortlich.

Das zweitwichtigste Technologiefeld mit 487 Patentanmeldungen ist "Transportieren I", das vor allem von den Branchen Fahrzeugindustrie, Elektroindustrie und Erdölindustrie dominiert wird. Das Technologiefeld "Elektrotechnik" liegt hinsichtlich der Patentierungen mit 486 Anmeldungen praktisch gleichauf. Es wird in besonderem Ausmaß von einer einzigen Branche, der Elektroindustrie, dominiert. 
Übersicht 2.2.5: Patentanmeldungen am österreichischen Patentamt durch österreichische Unternehmen (20 Beschäftigte und mehr) nach Branchen und Technologiefeldern, 1987 bis 1994

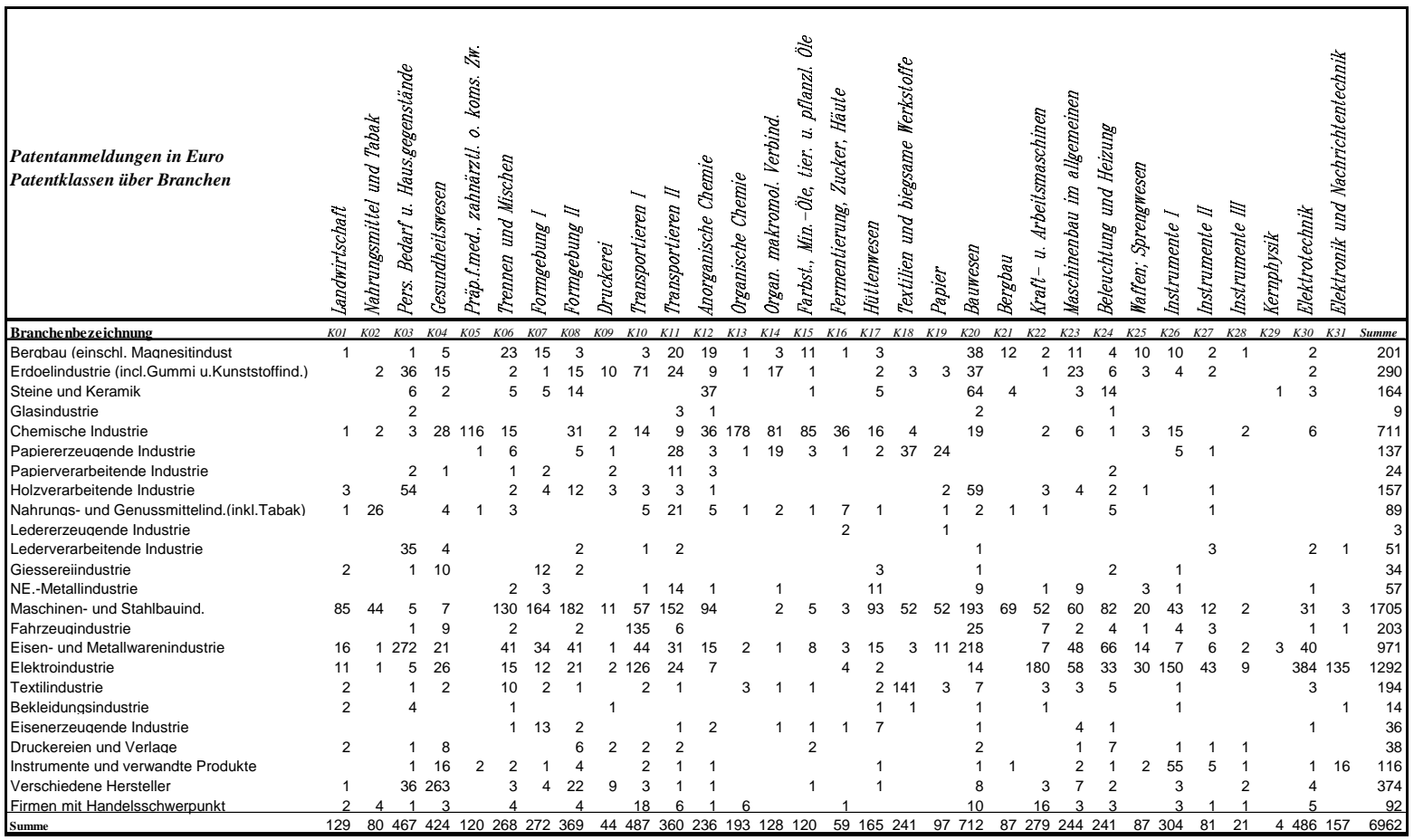

Q: Österreichisches Patentamt; Betriebs- und Produktdatenbank des Österreichischen Forschungszentrums Seibersdorf, zitiert aus Gassler et al. (1996). 


\section{Internationale Technologieströme}

Technologisches Wissen verbreitet sich nicht nur innerhalb eines Landes, sondern in zunehmendem Maße über nationale Grenzen hinweg. Vor allem für kleine, offene Volkswirtschaften ist der Import von Wissen seit langem eine entscheidende Determinante der wirtschaftlichen Entwicklung. Dies trifft umso mehr für Länder zu, die sich nicht im technologischen Spitzenfeld befinden. Die Diffusion von Technologie trug wesentlich zu der nach dem zweiten Weltkrieg beobachtbaren Konvergenz der Pro-Kopf-Einkommen in den OECD-Ländern bei. Gerade die Entwicklung Österreichs in der Nachkriegsperiode ist ein anschauliches Beispiel für einen erfolgreichen wirtschaftlichen und technologischen Aufholprozeß.

In offenen Wirtschaften, die miteinander durch internationalen Handel, Direktinvestitionen und Informationsströme verflochten sind, hängt die Entwicklung der wirtschaftlichen Leistungsfähigkeit - insbesondere der Produktivität - nicht nur von eigenen technologischen Anstrengungen ab. Die Fähigkeit von Ländern, im Ausland produziertes Wissen aufzunehmen und intelligent zu nutzen wird zu einem entscheidenden Faktor für die Realisierung eines angemessenen

Wirtschaftswachstums. Zur Herstellung dieser Fähigkeit bedarf es eigener Forschung und Entwicklung.

In Verbindung mit der zunehmenden Durchlässigkeit der Weltwirtschaft - Stichworte: Integration, Liberalisierung des Welthandels, Globalisierung - und die neue Rolle von Information im Wirtschaftsprozeß - Stichwort: Wissens- und Informationsgesellschaft erhöht sich die Bedeutung von internationalen Wissensströmen weiter. Zwischen den beiden wichtigsten Quellen des Produktivitätsfortschritts - im Land und im Ausland hervorgebrachtes technologisches Wissen - besteht kein Gegensatz. Vieles weist darauf hin, daß eigene F\&E-Anstrengungen und im Ausland hervorgebrachtes Wissen einander ergänzen. Information fließt nicht passiv. Es bedarf eigener Forschungs- und Innovationsanstrengungen, um in der Lage zu sein, internationale technologische Entwicklungen $\mathrm{zu}$ beobachten und $\mathrm{zu}$ bewerten, aufzunehmen und intelligent weiterzuentwickeln.

\subsection{Direktinvestitionen}

In den achtziger Jahren haben die Direktinvestitionen mehr als doppelt so rasch zugenommen wie die internationalen Handelsströme. Sie waren damit die dynamischste Komponente im weltweiten Prozeß der wirtschaftlichen Umstrukturierung. Unter dem Aspekt des Wissens- und Technologietransfers sind grundsätzlich sowohl aktive (hinausgehende) als auch passive (hereinkommende) Auslandsinvestitionen von Interesse. Aktive Auslandsinvestitionen, etwa in Form von Beteiligungen an und Übernahmen von Unternehmen im Ausland, können beispielsweise zum Aufbau technologischer Kompetenz in österreichischen Unternehmen beitragen. Passive Auslandsinvestitionen sind häufig mit 
Technologie- und Know-how-Tranfers aus dem Ausland verbunden. Das Ausmaß und die Zusammensetzung der passiven Auslandsinvestitionen lassen darüber hinaus Rückschlüsse auf die "Standortqualität" Österreichs zu.

Der als Relation zwischen den nach Österreich fließenden passiven Auslandsinvestitionen und den aktiven Auslandsinvestitionen aus dem OECD-Raum definierte Marktanteil Österreichs war in den achtziger Jahren auf $0,2 \%$ gefallen. Anfang der neunziger Jahre stieg dieser Anteil deutlich und schwankte bis Mitte der neunziger Jahre um etwa das dreifache Niveau. Im Jahr 1996 kam es - vor allem bedingt durch einige spektakuläre Übernahmen durch ausländische Unternehmen - neuerlich zu einer kräftigen Zunahme der österreichischen passiven Auslandsinvestitionen.

Gegen Ende der achtziger Jahre haben die aktiven Auslandsinvestitionen Österreichs drastisch zugenommen. Dieser Internationalisierungsschub fand ihren Niederschlag in einem kräftig steigenden Anteil der österreichischen aktiven Auslandsinvestitionen an den aktiven Auslandsinvestitionen der OECD. Einen vorläufigen Höhepunkt erreichte der österreichische Anteil mit 1,2\% im Jahr 1992. Seither ist dieser Anteil rückläufig (1995: $0,5 \%)$.

Es zeichnet sich ab, daß Österreich den zu Beginn der neunziger Jahre erreichten Anteil an den aktiven Auslandsinvestitionen nur sehr schwer auf Dauer halten wird können. Verantwortlich dafür sind u. a. die Anteilsverluste der österreichischen Direktinvestitionen in den mittel- und osteuropäischen Ländern. Der Anteil Österreichs an den neuen Direktinvestitionen in Ost-Mitteleuropa, der 1990 mehr als $41 \%$ betrug, sank bis 1995 auf $5,6 \%$. Insbesondere blieb eine maßgebliche Beteiligung österreichischer Unternehmen an den Privatisierungen im Bereich der öffentlichen Versorgungsunternehmen (Telekommunikation etc.) aus. Dieser Umstand ist auch unter technologiepolitischen Gesichtspunkten von hohem Interesse.

\subsection{Das technologische Profil des Außenhandels}

Mit dem Import von Waren bzw. Dienstleistungen wird auch technologisches Wissen importiert $^{9}$ ). Zum einen sind die international gehandelten Waren in einem unmittelbaren Sinn Träger von Information: Mit dem Warenhandel wird Wissen über Produkteigenschaften verbreitet; durch die Informations- und Lernprozesse, die mit dem Engagement im Außenhandel einhergehen, trägt dieser auch in indirekter Weise zur internationalen Verbreitung von Wissen bei. Zum anderen trägt der Import innovativer Kapital- und Vorleistungsgüter zur Erhöhung der Produktivität in der österreichischen Wirtschaft bei. Damit werden in Österreich Investitionen in Forschung und Technologie wirksam, die an-

\footnotetext{
${ }^{9}$ ) Der vorliegende Abschnitt stützt sich u. a. auf Peneder (1995) und Hutschenreiter - Peneder (1997). Dabei wurden Produktgruppen der Außenhandelsstatistik mehreren Klassen unterschiedlichen technologischen Anspruchsniveaus zugeordnet. Die dabei verwendete Klassifikation nach Legler (1982) und Schulmeister (1990) beruht im wesentlichen auf quantitativ meßbaren Größen der Faktoreinsatzmengen.
} 
derswo in der Welt getätigt wurden. Grenzüberschreitende Produktivitätseffekte von Forschung und Entwicklung werden als "internationale F\&E-Spillovers" bezeichnet ${ }^{10}$ ).

Zum anderen sind Unterschiede im technologischen Entwicklungsniveau auch eine Quelle nationaler Wettbewerbsvorteile im Export und haben daher unmittelbare Auswirkungen auf die in einer Volkswirtschaft erzielbaren Einkommen. Unternehmen mit technologisch anspruchsvollen Produkten können ihre Marktmacht leichter behaupten und über bessere Produktpreise höhere Faktoreinkommen erzielen. Länder, die am unteren Ende der "Qualitätsleiter" und auf weniger anspruchsvollen Produktmärkten operieren, müssen das Defizit an technologischen Qualifikationen durch geringere Faktorentgelte (niedrigere Löhne und Gewinne), einen größeren Einsatz natürlicher Ressourcen (Umwelt, Energie, Rohstoffe) oder eine intensivere Ausschöpfung von Produktivitätsreserven (Rationalisierungen etc.) kompensieren.

\section{Box 2: Klassifikation "typischer" Produktionstechnologien nach Legler und Schulmeister}

Die verschiedenen Merkmalskombinationen nach Legler (1982) wurden von Schulmeister (1990) zu verwandten Produktionstechnologien zusammengefaßt, wobei jede Produktgruppe nur einer Technologieklasse eindeutig zugeordnet werden darf. Die Klassifikation unterscheidet vier Hauptgruppen:

1. "Humankapitalintensiv" sind alle Produktgruppen mit besonders großem Einsatz von qualifizierten Arbeitskräften. Weiter wird unterschieden zwischen "Hochtechnologie" und "Gebrauchstechnologie", die wiederum jeweils überwiegend "sachkapital-" oder "arbeitsintensiv" sein können. Beispiele für die Kategorie "Hochtechnologie" sind Flugzeuge ebenso wie optische Instrumente, Prüf- und Analyseinstrumente, pharmazeutische Produkte oder bestimmte organische Chemikalien und Spezialkunststoffe. Als "Gebrauchstechnologie" werden Güter wie Papier- und Druckmaschinen, Nachrichtengeräte, Haushaltsgeräte, Geräte der Unterhaltungselektronik, Fotozubehör, Farben und Pigmente, Straßenfahrzeuge, organische und anorganische Chemikalien usw. eingestuft.

2. "Sachkapitalintensiv": Diese Obergruppe faßt nur jene Güter zusammen, deren Herstellung große Mengen des Produktionsfaktors Sachkapital erfordert, die gleichzeitig aber nicht als humankapital- oder ressourcenintensiv eingestuft wurden (z. B. Bandstahl, verschiedene Gewebe und Bodenbeläge).

3. "Arbeitsintensiv" sind nur jene Waren, die mit überdurchschnittlich hohem Einsatz des Faktors Arbeit hergestellt werden, aber gleichzeitig weder humankapital- noch ressourcenintensiv sind (z. B. Lederwaren, Textilien, Bekleidung, Schuhe, Möbel, Papierwaren sowie Spiel- und Sportgeräte).

\footnotetext{
$\left.{ }^{10}\right)$ Siehe Hutschenreiter (1995).
} 
4. "Ressourcenintensiv": Diese Obergruppe enthält nur Güter mit hohem Verbrauch an agrarischen oder mineralischen Rohstoffen und wird weiter nach dem Grad des Verbrauchs in "schwach" und "stark" ressourcenintensive Güter unterteilt. Letztere umfassen auch einige Produktgruppen, die vor allem ressourcenintensiv sind, aber (obwohl in geringerem Maße) überdurchschnittlich viel Humankapital einsetzen. Beispiele dieser Obergruppe sind Grundstoffe organischer Chemikalien, Düngemittel, Eisen und Stahl sowie Papier und Pappe (alle "stark ressourcenintensiv") sowie Baumaterialien, Zement, Glas, Garne oder verschiedene Holzwaren ("schwach ressourcenintensiv"). 
Im langfristigen Vergleich wächst das Volumen des Außenhandels (Exporte und Importe von Industriewaren auf Dollarbasis) der OECD-Länder bei technologieintensiven Produktgruppen am schnellsten: Die durchschnittliche jährliche Wachstumsrate betrug von 1970 bis 1994 für humankapitalintensive Güter 12,1\%, für arbeitsintensive Güter 11,4\%, für sachkapitalintensive Güter (die weder humankapital- noch ressourcenintensiv sind) 8,9\% und für ressourcenintensive Güter 8,8\%. Innerhalb der humankapitalintensiven Güter weist die Hochtechnologie $(+14,1 \%)$ langfristig ein deutlich höheres Marktwachstum auf als das Segment der Gebrauchstechnologie $(+11,6 \%)$, dessen Dynamik beständig nachläßt.

Im langfristigen Vergleich wachsen die internationalen Märkte für technologisch anspruchsvolle Güter rascher als jene für Waren mit geringem Technologiegehalt. Besonders der Handel mit Gütern der Hochtechnologie weist hohe Zuwachsraten auf. Der Anteil arbeitsintensiver und ressourcenintensiver Waren ist rückläufig.

Die unterschiedliche Wachstumsdynamik schlägt sich in Veränderungen der Außenhandelsstruktur nach Technologieklassen nieder (Übersicht 3.2.1): So stieg der Anteil humankapitalintensiver Güter am Export und Import der OECD-Länder in den siebziger Jahren zwar nur mäßig, seit Anfang der achtziger Jahre aber kräftig (1970 Exportanteil 52,9\%, Importanteil 47,3\%; 1980 54,7\% bzw. 47,8\%, 1994 63,7\% bzw. 55,7\%). Der überwiegende Teil des Marktwachstums humankapitalintensiver Güter ist auf die rasche Expansion des Handels mit Gütern der Hochtechnologie zurückzuführen, während sich der Anteil der Gebrauchstechnologie über mehr als zwei Jahrzehnte nur wenig verändert hat (1994: Hochtechnologie Exportanteil 17,5\%, Importanteil 16,5\%, Gebrauchstechnologie 38,1\% bzw. 32,4\%).

Sachkapitalintensive Güter, die weder humankapitalintensiv noch ressourcenintensiv sind, spielen im Außenhandel der OECD mit Industriewaren eine geringe und weiterhin abnehmende Rolle (Export 1970 3,4\%, 1994 2,0\%, Import 3,5\% bzw. 1,9\%). Der Exportanteil arbeitsintensiver Güter, die weder humankapital- noch ressourcenintensiv sind, war seit 1970 relativ stabil, zuletzt zeichnet sich aber ein Trend zur Produktionsverlagerung aus der OECD in wirtschaftlich weniger entwickelte Regionen ab. Der Importanteil dieser Kategorie stieg in den siebziger Jahren und seit 1990. Er war 1994 mit 26,3\% deutlich höher als der Anteil am OECD-Export (18,7\%). Das Gewicht ressourcenintensiver Produkte im Außenhandel mit Industriewaren sinkt seit 1970 beständig (1994 Exportanteil 11,8\%, Importanteil 12,4\%). 
Übersicht 3.2.1: Außenhandelsstruktur der OECD-Länder nach Technologieklassen (SITC 5 bis 8)

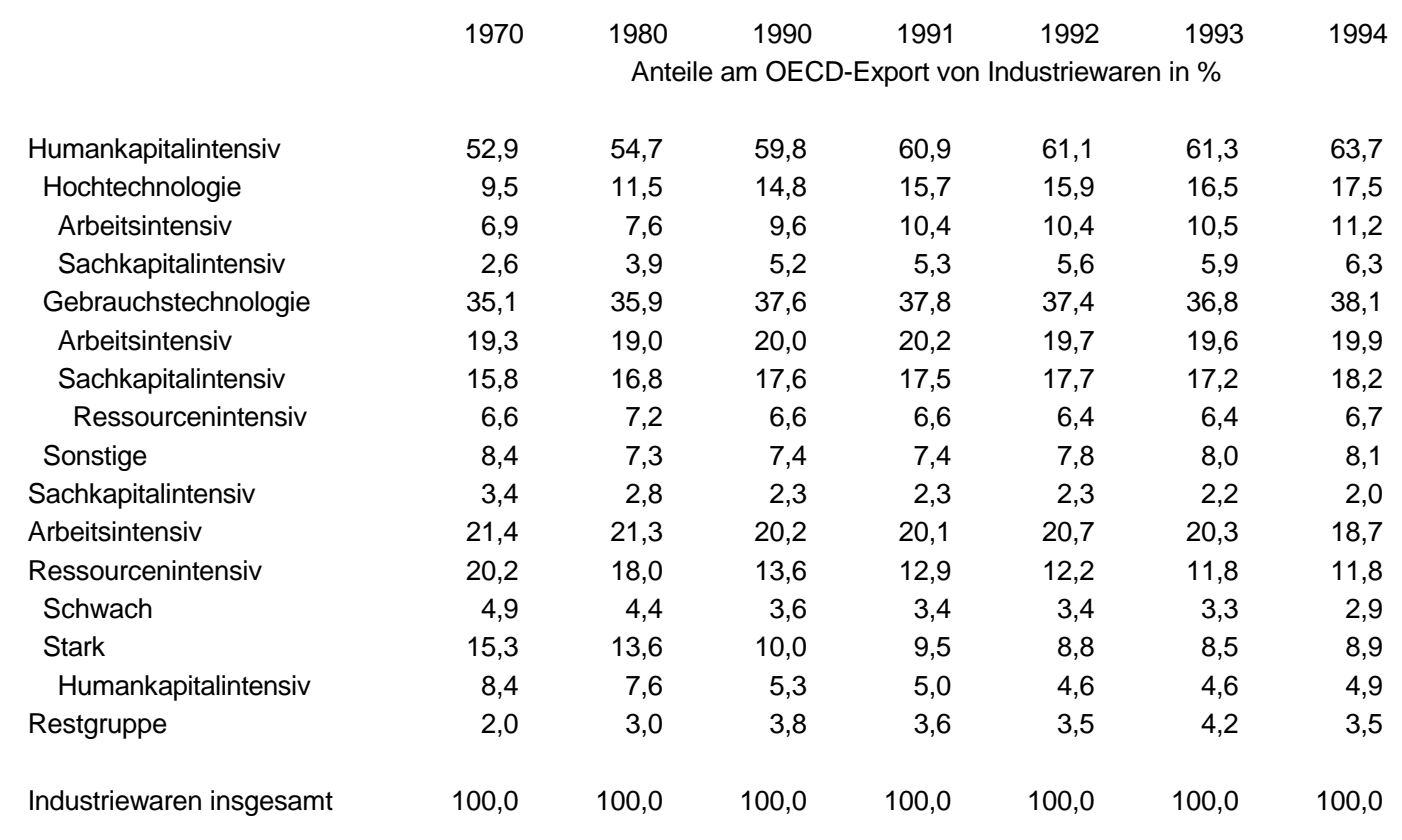

Humankapitalintensiv

Hochtechnologie

Arbeitsintensiv

Sachkapitalintensiv

Gebrauchstechnologie

Arbeitsintensiv

Sachkapitalintensiv

Ressourcenintensiv

Sonstige

Sachkapitalintensiv

Arbeitsintensiv

Ressourcenintensiv

Schwach

Stark

Humankapitalintensiv

Restgruppe

Industriewaren insgesamt

9,0

6,4

2,6

31,4

16,6

14,8

6,3

6,9

3,5

22,9

23,7

5,4

18,2

8,5

2,6

100,0
Anteile am OECD-Import von Industriewaren in \%

$\begin{array}{rrrrrr}47,8 & 54,7 & 55,6 & 55,5 & 55,4 & 55,7 \\ 11,2 & 14,2 & 15,1 & 15,3 & 16,1 & 16,5 \\ 7,2 & 8,5 & 9,1 & 8,9 & 9,1 & 9,5 \\ 4,0 & 5,7 & 6,0 & 6,4 & 7,0 & 7,0 \\ 30,8 & 34,1 & 34,0 & 33,6 & 32,5 & 32,4 \\ 15,5 & 17,6 & 17,6 & 17,2 & 16,7 & 17,0 \\ 15,3 & 16,5 & 16,4 & 16,4 & 15,8 & 15,5 \\ 6,2 & 6,0 & 5,9 & 5,8 & 5,6 & 5,5 \\ 5,8 & 6,5 & 6,5 & 6,6 & 6,8 & 6,7 \\ 3,0 & 2,3 & 2,2 & 2,2 & 2,0 & 1,9 \\ 25,7 & 24,7 & 25,2 & 26,0 & 26,4 & 26,3 \\ 19,6 & 14,4 & 13,4 & 12,7 & 12,1 & 12,4 \\ 4,9 & 4,0 & 3,8 & 3,8 & 3,7 & 3,6 \\ 14,7 & 10,4 & 9,6 & 8,9 & 8,4 & 8,9 \\ 7,0 & 5,0 & 4,7 & 4,4 & 4,2 & 4,5 \\ 4,0 & 3,9 & 3,6 & 3,6 & 4,1 & 3,7 \\ & & & & & \\ 100,0 & 100,0 & 100,0 & 100,0 & 100,0 & 100,0\end{array}$

Q: Hutschenreiter - Peneder (1997), Klassifikation nach Legler (1982), Schulmeister (1990). 1970: SITC rev. 1; 1980 : SITC rev. 2; 1990 bis 1994: SITC rev. 3. 
Österreichs weist ein strukturelles Defizit im Außenhandel mit Industriewaren auf. Im Jahr 1994 betrug dieses Defizit 75,8 Mrd. S (Übersicht 3.2.2). Dieses Handelsbilanzdefizit rückt durch die rückläufige Entwicklung im Bereich des Tourismus wieder stärker ins Zentrum wirtschaftspolitischer Aufmerksamkeit. In den neunziger Jahren nahm vor allem das Außenhandelsdefizit bei (nicht humankapitalintensiven) arbeitsintensiven Produktgruppen stark zu (1989: 17,9 Mrd. S, 1994: 35,4 Mrd. S). Diese Entwicklung ist für sich genommen nicht weiter besorgniserregend, da sie einem Strukturwandel im Außenhandel gemäß sich ändernden komparativen Vorteilen in einer sich globalisierenden Wirtschaft entspricht. Allerdings entsteht der größte Teil des Außenhandelsdefizits mit Industriewaren nach wie vor im Bereich humankapitalintensiver Produktgruppen. Im Jahr 1994 betrug das Handelsbilanzdefizit bei humankapitalintensiven Produktgruppen 57,1 Mrd. S, wovon 23 Mrd. S auf Güter der Hochtechnologie entfielen.

Österreich hat ein strukturelles Defizit im Außenhandel mit Industriewaren. Ein großer Teil dieses Defizits entsteht im Bereich der humankapitalintensiven Güter. Bei Gütern der Hochtechnologie erreichte das Außenhandelsdefizit 1994 rund 23 Mrd. S.

Österreich ist es nicht gelungen, die zu erwartende Zunahme des Außenhandelsdefizits bei arbeits- und sachkapitalintensiven Produktgruppen bzw. die abnehmenden Überschüsse bei ressourcenintensiven Produkten durch eine Verbesserung der Außenhandelsposition bei humankapitalintensiven Produkten zu kompensieren.

Der Grad der Exportspezialisierung Österreichs auf technologisch anspruchsvolle Güter ist für ein entwickeltes Industrieland nach wie vor außerordentlich gering: 1994 machten humankapitalintensive Güter 52,3\% der gesamten Exporte von Industriewaren aus, Produkte der Hochtechnologie nur 8,2\% (Übersicht 3.2.3). In der OECD insgesamt liegen diese Anteile hingegen wie gezeigt bei $63,7 \%$ und $17,5 \%$.

Österreich ist mit einem Anteil humankapitalintensiver Güter am gesamten Industriewarenexport von 52,3\% und einem Hochtechnologieanteil von 8,2\% deutlich weniger auf technologisch hochwertige Produkte spezialisiert als vergleichbare kleine offene Volkswirtschaften in Europa wie z. B. die Schweiz und Schweden. Der österreichische Außenhandel zeigt eine ausgeprägte "Technologielücke": Sowohl in den OECD- als auch in den EU-Ländern entfällt auf Hochtechnologie ein mehr als doppelt so hoher Exportanteil wie in Österreich.

Besonders interessant ist für Österreich ein Vergleich mit hochentwickelten kleinen offenen Volkswirtschaften wie z. B. der Schweiz und Schweden oder einem dynamischen Nachzügler wie Finnland (Abbildung 3.2.1). Gemessen am Anteil humankapitalintensiver Güter am gesamten Export von Industriewaren (1994 57,5\% bzw. 59,7\%) weisen die Schweiz und Schweden ein günstigeres technologisches Spezialisierungsprofil auf als Österreich (52,3\%) und Finnland (44,9\%). Auch in der Exportspezialisierung mit Gütern der Hochtechnologie rangieren die Schweiz $(18,0 \%)$ und Schweden $(10,4 \%)$ vor Österreich 
$(8,2 \%)$ und Finnland (6,7\%). In der Kategorie der Gebrauchstechnologien liegt Schweden $(41,7 \%)$ vor der Schweiz (34,2\%), Österreich $(33,3 \%)$ und Finnland $(30,9 \%)$.

Übersicht 3.2.2: Österreichs Handelsbilanz nach Technologieklassen

\begin{tabular}{|c|c|c|c|c|c|c|}
\hline & 1989 & 1990 & 1991 & 1992 & 1993 & 1994 \\
\hline Humankapitalintensiv & $-51.467,9$ & $-48.522,0$ & $-57.733,2$ & $-56.785,8$ & $-46.538,0$ & $-57.113,0$ \\
\hline Hochtechnologie & $-21.801,4$ & $-19.123,5$ & $-20.641,4$ & $-20.269,7$ & $-18.982,3$ & $-22.967,0$ \\
\hline Arbeitsintensiv & $-10.517,2$ & $-7.547,3$ & - $8.553,9$ & $-9.006,0$ & $-6.286,5$ & - $8.810,3$ \\
\hline Sachkapitalintensiv & $-11.284,2$ & $-11.576,1$ & $-12.087,6$ & $-11.263,6$ & $-12.695,8$ & $-14.156,7$ \\
\hline Gebrauchstechnologie & $-39.990,3$ & $-39.942,1$ & $-47.064,4$ & $-49.268,9$ & $-36.357,9$ & $-44.618,9$ \\
\hline Arbeitsintensiv & - $3.667,4$ & $1.537,7$ & $-2.666,6$ & $-\quad 3.602,6$ & 851,3 & $-\quad 2.444,6$ \\
\hline Sachkapitalintensiv & $-36.323,0$ & $-41.479,8$ & $-44.397,8$ & $-45.666,2$ & $-37.209,2$ & $-42.174,3$ \\
\hline Davon: Ressoursenintensiv & $1.208,4$ & $-\quad 2.804,6$ & $-\quad 2.703,0$ & - $4.357,4$ & $-4.453,5$ & - $5.688,4$ \\
\hline Sonstige & $10.323,8$ & $10.543,6$ & $9.972,7$ & $12.752,8$ & $8.802,2$ & $10.473,0$ \\
\hline Sachkapitalintensiv & 270,8 & 313,3 & 325,7 & 325,6 & 644,8 & 810,6 \\
\hline Arbeitsintensiv & $-17.875,6$ & $-23.980,1$ & $-27.933,0$ & $-26.229,2$ & $-28.522,9$ & $-35.383,7$ \\
\hline Ressourcenintensiv & $24.963,9$ & $26.056,2$ & $23.895,7$ & $21.656,9$ & $19.557,7$ & $18.386,2$ \\
\hline Schwach & $5.876,2$ & $6.474,6$ & $5.066,2$ & $4.911,3$ & $3.195,2$ & $2.924,9$ \\
\hline Stark & $19.087,8$ & $19.581,7$ & $18.829,5$ & $16.745,5$ & $16.362,6$ & $15.461,2$ \\
\hline Davon: Humankapitalintensiv & $8.707,2$ & $8.754,7$ & $7.474,0$ & $7.321,9$ & $8.440,7$ & $8.163,3$ \\
\hline Restgruppe & 250,9 & 531,5 & 429,5 & 412,3 & 514,5 & 739,4 \\
\hline Industriewaren insgesamt & $-44.387,2$ & $-46.818,7$ & $-62.723,5$ & $-62.274,1$ & $-56.804,0$ & $-75.813,4$ \\
\hline
\end{tabular}

Q: WIFO, Klassifikation nach Legler (1982), Schulmeister (1990). SITC rev. 3.

Abbildung 3.2.1: Österreichs Außenhandelsspezialisierung im Vergleich mit anderen kleinen offenen Volkswirtschaften
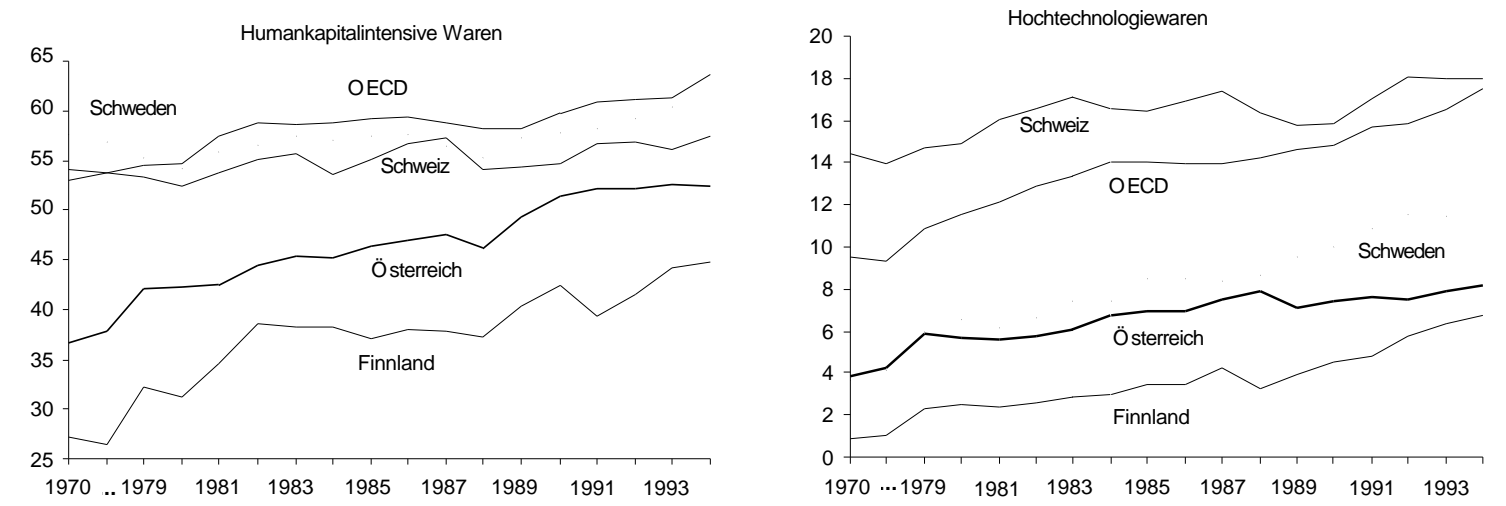

Q: Hutschenreiter - Peneder (1997), 1970 - 1979: SITC rev.1; 1980 - 1989; SITC rev.2; 1989 - 1994: SITC rev.3. 
Übersicht 3.2.3: Österreichs Außenhandelsspezialisierung nach Technologieklassen SITC 5 bis 8

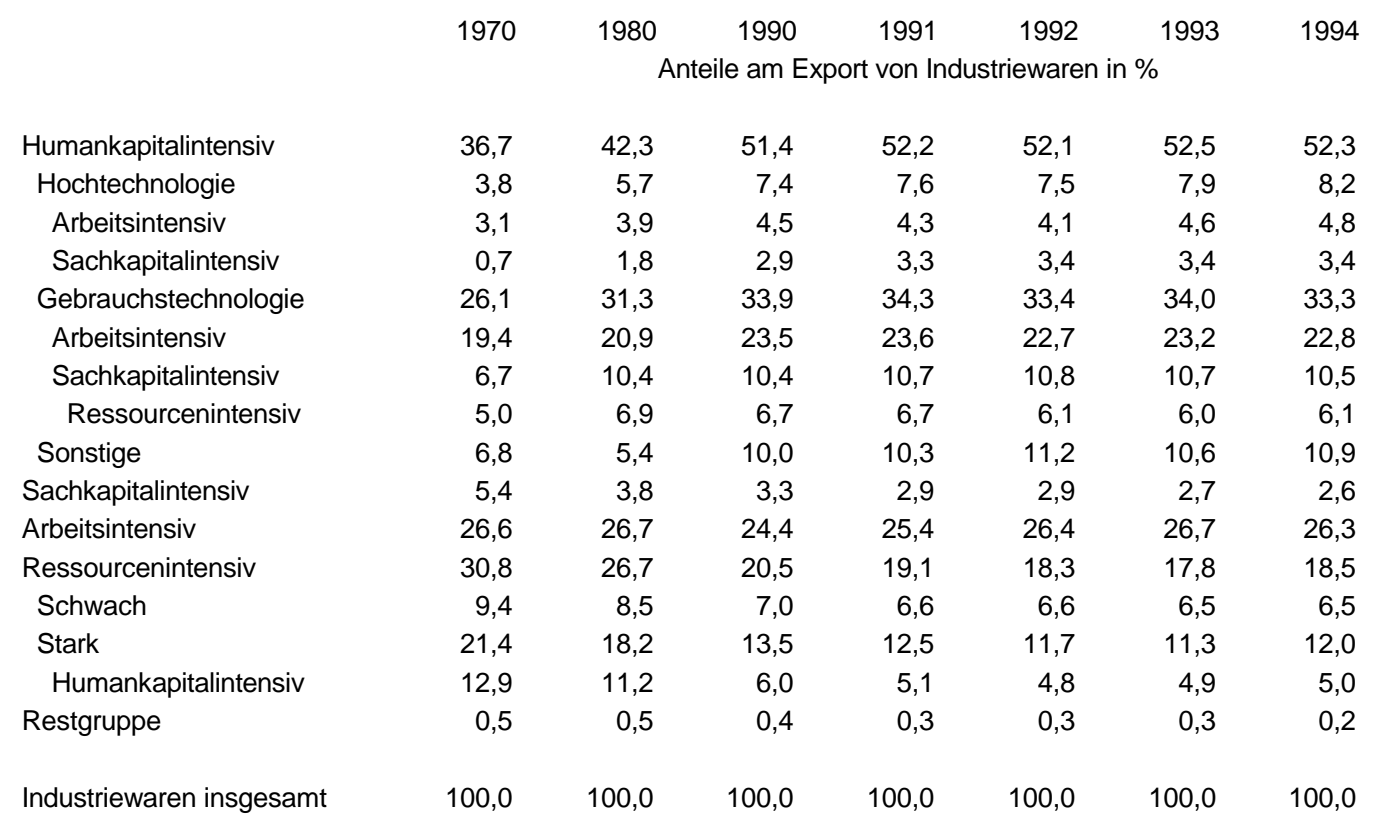

\begin{tabular}{|c|c|c|c|c|c|c|c|}
\hline Humankapitalintensiv & 53,2 & 51,2 & 56,5 & 57,2 & 56,9 & 55,9 & 55,6 \\
\hline Hochtechnologie & 7,3 & 8,5 & 10,7 & 10,8 & 10,5 & 10,9 & 11,3 \\
\hline Arbeitsintensiv & 4,6 & 4,8 & 5,7 & 5,4 & 5,3 & 5,3 & 5,8 \\
\hline Sachkapitalintensiv & 2,7 & 3,7 & 5,1 & 5,3 & 5,2 & 5,6 & 5,5 \\
\hline Gebrauchstechnologie & 40,3 & 37,6 & 39,0 & 39,4 & 39,0 & 37,5 & 36,9 \\
\hline Arbeitsintensiv & 20,8 & 19,6 & 20,9 & 21,2 & 20,6 & 20,3 & 20,0 \\
\hline Sachkapitalintensiv & 19,5 & 18,1 & 18,1 & 18,2 & 18,4 & 17,2 & 16,8 \\
\hline Ressourcenintensiv & 8,9 & 7,5 & 6,6 & 6,4 & 6,2 & 6,2 & 6,3 \\
\hline Sonstige & 5,6 & 5,1 & 6,8 & 7,1 & 7,3 & 7,6 & 7,4 \\
\hline Sachkapitalintensiv & 4,4 & 3,8 & 2,9 & 2,6 & 2,6 & 2,6 & 2,4 \\
\hline Arbeitsintensiv & 22,8 & 28,4 & 27,1 & 27,8 & 28,3 & 29,4 & 29,1 \\
\hline Ressourcenintensiv & 19,4 & 16,1 & 13,1 & 11,9 & 11,9 & 11,7 & 12,6 \\
\hline Schwach & 8,0 & 6,8 & 5,0 & 4,8 & 4,8 & 5,0 & 5,1 \\
\hline Stark & 11,4 & 9,3 & 8,1 & 7,2 & 7,0 & 6,6 & 7,5 \\
\hline Humankapitalintensiv & 5,7 & 4,7 & 3,6 & 3,0 & 2,8 & 2,6 & 2,8 \\
\hline Restgruppe & 0,2 & 0,5 & 0,4 & 0,4 & 0,3 & 0,3 & 0,3 \\
\hline Industriewaren insgesamt & 100,0 & 100,0 & 100,0 & 100,0 & 100,0 & 100,0 & 100,0 \\
\hline
\end{tabular}

Anteile am Import von Industriewaren in \% 
Österreich erzielt im Handel mit humankapitalintensiven Waren sowohl der Hochtechnologie als auch der Gebrauchstechnologie deutlich geringere Unit Values (Erlöse je Mengeneinheit) als andere kleine offene Volkswirtschaften wie z. B. die Schweiz, Schweden und auch Finnland.

Österreichs Exporte an humankapitalintensiven Waren erzielen deutlich geringere Unit Values (Exporterlös je Mengeneinheit) als jene der Schweiz, Schwedens und Finnlands (Übersicht 3.2.4; 1994 Österreich 5,0 \$ je kg, Finnland 12,0 \$ je kg, Schweden 11,5 \$ je $\mathrm{kg})$. Die Schweiz nimmt hier mit 13,8 \$ je $\mathrm{kg}$ eine internationale Spitzenstellung ein. Im Export von Hochtechnologie erwirtschaftet Schweden die höchsten Unit Values unter den vier Ländern (1994 64,3 \$ je kg, Schweiz 42,1 \$ je kg, Finnland 37,2 \$ je kg), Österreich $(16,7 \$$ je $\mathrm{kg})$ bleibt abermals deutlich zurück. Im Export von Gebrauchstechnologie betragen die entsprechenden Unit Values $12,4 \$$ je $\mathrm{kg}$ in der Schweiz, 11,9\$ je kg in Schweden, 11,1 \$ je kg in Finnland und nur 4,0 \$ je kg in Österreich.

Innerhalb der gleichen Technologieklassen weisen also österreichische Exporte ein Qualitätsdefizit gegenüber vergleichbaren offenen Volkswirtschaften auf. Gegenüber einem Aggregat von 13 Industrieländern ${ }^{11}$ ) sind diese Unterschiede weniger ausgeprägt.

Das ungünstige Spezialisierungsmuster im österreichischen Außenhandel unterliegt einem Strukturwandel zugunsten technologisch anspruchsvollerer Produkte, der allerdings nur relativ langsam vorankommt (Übersicht 3.2.3). So stieg der Anteil humankapitalintensiver Waren am gesamten Export von Industriewaren von 36,7\% (1970) auf 52,3\% (1994). Diese Entwicklung geht sowohl auf eine zunehmende Bedeutung der Hochtechnologie (1970 3,8\%, 1994 8,2\%) als auch der Gebrauchstechnologie zurück (1970 26,1\%, 1994 33,3\%). Der Strukturwandel geht vor allem zu Lasten ressourcenintensiver Produktgruppen (Exportanteil 1970 30,8\%, 1994 18,5\%) und sachkapitalintensiver Güter (die weder humankapital- noch ressourcenintensiv produziert werden; 1970 5,4\%, 1994 2,6\%). Der Anteil arbeitsintensiver Güter (die weder humankapital- noch kapitalintensiv erzeugt werden) blieb hingegen stabil (1970 26,6\%, 1994 26,3\%).

\footnotetext{
${ }^{11}$ ) Die Auswahl der Länder erfolgte nach der Verfügbarkeit der zur Ermittlung der Unit Values erforderlichen Daten.
} 
Table 3.2.4: Unit Values im Außenhandel 1994

\begin{tabular}{|c|c|c|c|c|c|c|c|c|c|c|}
\hline & Öster & eich & & & Schw & den & Finn & and & Deuts & hland \\
\hline & Export & Import & Export & Import & Export & Import & Export & Import & Export & Import \\
\hline & $\$ \mathrm{je}$ & & & & $\$ \mathrm{je}$ & & $\$ \mathrm{je}$ & & $\$ \mathrm{je}$ & \\
\hline Humankapitalintensiv & 5,034 & 5,737 & 13,841 & 6,566 & 11,548 & 6,381 & 11,988 & 7,092 & 6,425 & 5,884 \\
\hline Hochtechnologie & 16,704 & 20,974 & 42,110 & 18,337 & 64,272 & 23,924 & 37,150 & 39,470 & 20,133 & 28,573 \\
\hline Arbeitsintensiv & 21,071 & 25,504 & 138,368 & 59,793 & 206,251 & 14,370 & 49,648 & 38,919 & 46,467 & 58,737 \\
\hline Sachkapitalintensiv & 7,508 & 15,154 & 15,664 & 10,096 & 44,648 & 46,343 & 30,567 & 41,105 & 6,477 & 12,865 \\
\hline Gebrauchstechnologie & 3,963 & 4,745 & 12,378 & 5,334 & 11,928 & 5,653 & 11,126 & 5,739 & 5,454 & 4,245 \\
\hline Arbeitsintensiv & 12,407 & 12,560 & 30,674 & 16,636 & 12,505 & 15,946 & 12,034 & 17,734 & 17,940 & 12,212 \\
\hline Sachkapitalintensiv & 1,601 & 2,723 & 6,168 & 3,214 & 11,149 & 2,617 & 6,665 & 2,721 & 3,286 & 2,427 \\
\hline Ressourcenintensiv & 1,091 & 1,280 & 5,840 & 1,842 & 2,200 & 1,487 & 3,214 & 1,718 & 1,536 & 1,222 \\
\hline Sonstige & 9,769 & 7,298 & 13,133 & 8,282 & 9,079 & 5,028 & 8,761 & 4,179 & 6,482 & 6,122 \\
\hline Sachkapitalintensiv & 2,311 & 3,371 & 6,033 & 2,878 & 2,308 & 1,881 & 2,321 & 3,691 & 4,245 & 3,324 \\
\hline Arbeitsintensiv & 6,297 & 7,545 & 11,371 & 9,184 & 4,315 & 5,419 & 2,956 & 7,279 & 7,503 & 7,319 \\
\hline Ressourcenintensiv & 0,836 & 0,873 & 2,599 & 1,106 & 0,839 & 0,871 & 0,751 & 0,910 & 0,842 & 0,647 \\
\hline Schwach & 1,127 & 0,829 & 2,485 & 1,056 & 0,486 & 0,867 & 0,944 & 0,877 & 1,155 & 0,541 \\
\hline Stark & 0,733 & 0,906 & 2,667 & 1,134 & 1,352 & 0,873 & 0,738 & 0,925 & 0,757 & 0,712 \\
\hline Humankapitalintensiv & 0,569 & 0,548 & 6,200 & 0,918 & 1,145 & 0,679 & 0,812 & 0,624 & 0,558 & 0,507 \\
\hline Restgruppe & 1,674 & 0,894 & 6,446 & 4,342 & . & 1,334 & 1,095 & 1,814 & 10,629 & 10,888 \\
\hline Industriewaren insgesamt & 2,581 & 3,384 & 8,637 & 4,058 & 4,012 & 3,524 & 1,556 & 3,865 & 3,708 & 2,772 \\
\hline
\end{tabular}


Von geringem Niveau aus vollzieht sich ein Strukturwandel der österreichischen Exporte zugunsten humankapitalintensiver Güter sowohl der Hoch-als auch der Gebrauchstechnologie, der sich in internationalen Marktanteilsgewinnen äußert.

Der Strukturwandel der österreichischen Exporte zugunsten humankapitalintensiver Produkte ist nicht ausschließlich auf die international höhere Wachstumsdynamik technologisch anspruchsvoller Güter zurückzuführen:

- Der Beitrag technologisch anspruchsvoller Produkte zum österreichischen Export wächst rascher als ihr Beitrag zum Import. Der Grad der Außenhandelsspezialisierung auf humankapitalintensive Güter (RCA-Wert) ${ }^{12}$ ) stieg entsprechend von -0,37 (1970) auf $-0,19$ (1980) und $-0,06$ (1994). Der RCA-Wert für Hochtechnologie verbesserte sich ebenfalls von $-0,66$ (1970) und $-0,41$ (1980) auf $-0,32$ (1994) und jener für die Gebrauchstechnologie von $-0,44$ (1970) und $-0,19$ (1980) auf $-0,10$ (1994).

- Der Exportanteil technologisch anspruchsvoller Produktgruppen wächst in Österreich rascher als im Durchschnitt der OECD-Länder. Österreich verzeichnete daher im Außenhandel mit humankapitalintensiven Gütern Marktanteilsgewinne von 0,96\% (1970) auf 1,22\% (1980), 1,62\% (1990) und zuletzt 1,55\% (1994). Diese Marktanteilsgewinne gehen sowohl auf die Hochtechnologie (Marktanteil 1994 0,89\%) als auch auf die Gebrauchstechnologie (1,65\%) zurück (Übersicht 3.2.5). Zum Vergleich: Am Außenhandel der OECD-Länder mit Industriewaren insgesamt hielt Österreich 1994 einen Marktanteil von 1,89\%.

Die Ergebnisse der Analyse belegen nicht nur, daß sich die Struktur der österreichischen Exporte langfristig zugunsten technologisch höherwertiger Produkte verändert, sondern auch daß Österreich in diesem Segment Marktanteile gewonnen hat. Gleichzeitig ist der Anteil technologisch anspruchsvoller Produktgruppen im internationalen Vergleich aber nach wie vor gering.

\subsection{Technologische Zahlungsbilanz}

Analysen des Warenhandels erlauben eine Abschätzung des Umfangs der in tangiblen Waren gebundenen internationalen Technologieströme. Mit der Technologischen

\footnotetext{
${ }^{12}$ ) Der RCA-Wert ("revealed comparative advantage") wird in logarithmierter Form aus dem Verhältnis der Exporte zu den Importen einer bestimmten Produktgruppe im Zähler und dem gleichen Verhältnis für Industriewaren insgesamt im Nenner berechnet:
}

$R C A_{i}=\ln \left(\frac{X_{i} / M_{i}}{X_{T} / M_{T}}\right)$,

$i \ldots$ Technologieklasse, $T \ldots$ Industriewaren insgesamt). Ein Wert größer als Null steht für eine positive Außenhandelsspezialisierung auf diese Produktgruppe, ein Wert kleiner als Null für eine negative Spezialisierung. 
Zahlungsbilanz hingegen wird versucht, die Stellung eines Landes im internationalen Handel mit technischem Wissen und mit Dienstleistungen mit technologischem Inhalt zu ermitteln. In der Technologischen Zahlungsbilanz werden Zahlungsströme zwischen Staaten erfaßt, die mit dem Kauf und Verkauf wissenschaftlichen oder technischen Wissens zusammenhängen.

Gemäß den Richtlinien der OECD umfaßt der in der Technologischen Zahlungsbilanz abgebildete Technologiehandel die folgenden beiden Kategorien von Zahlungsströmen:

- Transaktionen in Zusammenhang mit industriellem Eigentum (Patente, Lizenzen, Techniken, Prozesse, Know-how, Design, Muster);

- Transaktionen in Zusammenhang mit Dienstleistungen mit technischem Inhalt und intellektuellen Dienstleistungen (Engineering-Studien, technische Hilfsleistungen, F\&EDienstleistungen etc.).

Die größte Bedeutung kommt dabei Einnahmen und Ausgaben in Verbindung mit Patenten und Erfindungen und der Lizensierung von Patenten und Know-how zu.

Österreich weist ein strukturelles Defizit in der Technologischen Zahlungsbilanz (Überhang der Ausgaben über die Einnahmen) aus. Im Jahr 1995 betrugen die Ausgaben 5,369 Mrd. S und die Einnahmen 1,478 Mrd. S. Die Deckungsquote (Verhältnis von Einnahmen zu Ausgaben) schwankt langfristig (seit Anfang der siebziger Jahre) um einen leicht steigenden Trend. In den letzten Jahren deckten die Einnahmen aus dem Technologiehandel ein Viertel bis ein Drittel der entsprechenden Ausgaben. Im letztverfügbaren Jahr (1995) betrug Österreichs Deckungsquote lediglich 25\%.

Übersicht 3.2.5: Österreichs Marktanteile am Export der OECD nach Technologieklassen SITC 5 bis 8

$\begin{array}{llllllll}\text { Humankapitalintensiv } & 0,96 & 1,22 & 1,62 & 1,56 & 1,58 & 1,45 & 1,55 \\ \text { Hochtechnologie } & 0,55 & 0,78 & 0,95 & 0,88 & 0,87 & 0,82 & 0,89 \\ \text { Arbeitsintensiv } & 0,62 & 0,81 & 0,89 & 0,75 & 0,72 & 0,73 & 0,81 \\ \text { Sachkapitalintensiv } & 0,38 & 0,72 & 1,06 & 1,13 & 1,13 & 0,96 & 1,02 \\ \text { Gebrauchstechnologie } & 1,03 & 1,38 & 1,70 & 1,65 & 1,65 & 1,57 & 1,65 \\ \text { Arbeitsintensiv } & 1,40 & 1,73 & 2,21 & 2,12 & 2,12 & 2,01 & 2,16 \\ \text { Sachkapitalintensiv } & 0,59 & 0,98 & 1,12 & 1,10 & 1,13 & 1,06 & 1,09 \\ \quad \text { Ressourcenintensiv } & 1,06 & 1,52 & 1,92 & 1,83 & 1,74 & 1,60 & 1,72 \\ \text { Sonstige } & 1,13 & 1,17 & 2,55 & 2,54 & 2,67 & 2,25 & 2,54 \\ \text { Sachkapitalintensiv } & 2,20 & 2,15 & 2,63 & 2,31 & 2,28 & 2,13 & 2,42 \\ \text { Arbeitsintensiv } & 1,73 & 1,98 & 2,28 & 2,29 & 2,35 & 2,23 & 2,67 \\ \text { Ressourcenintensiv } & 2,12 & 2,34 & 2,85 & 2,69 & 2,77 & 2,56 & 2,96 \\ \text { Schwach } & 2,66 & 3,04 & 3,71 & 3,52 & 3,58 & 3,33 & 4,25 \\ \text { Stark } & 1,94 & 2,11 & 2,54 & 2,39 & 2,45 & 2,26 & 2,54 \\ \quad \text { Humankapitalintensiv } & 2,14 & 2,31 & 2,15 & 1,85 & 1,92 & 1,83 & 1,96 \\ \text { Restgruppe } & 0,36 & 0,24 & 0,18 & 0,15 & 0,15 & 0,11 & 0,13 \\ & & & & & & & \\ \text { Industriewaren insgesamt } & 1,39 & 1,58 & 1,89 & 1,81 & 1,85 & 1,70 & 1,89\end{array}$


Q: Hutschenreiter - Peneder (1997), Klassifikation nach Legler (1982), Schulmeister (1990). 1970: SITC rev. 1; 1980: SITC rev. 2; 1990 bis 1994: SITC rev. 3.

Übersicht 3.3.1: Technologische Zahlungsbilanz von OECD-Ländern, 1994¹)

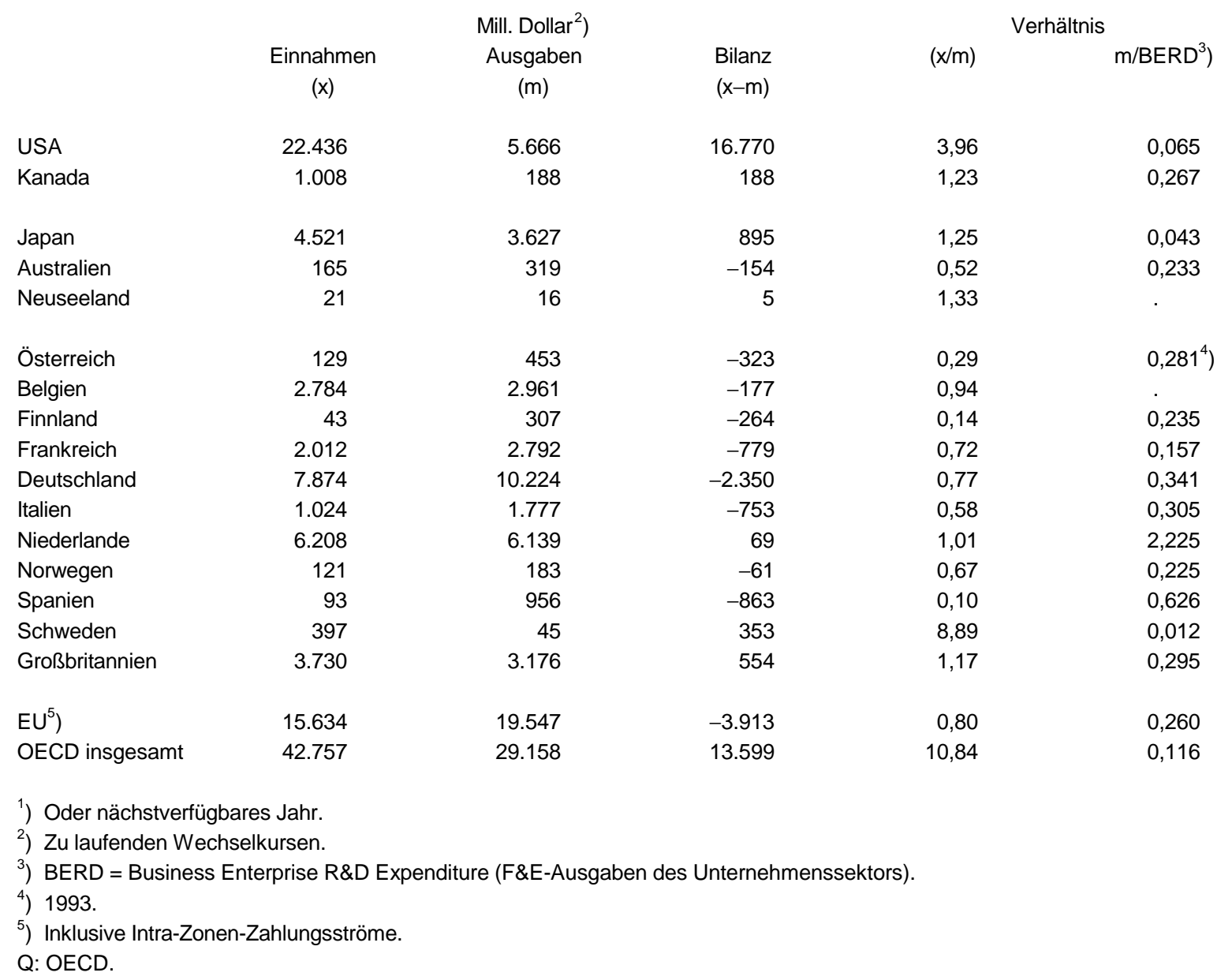

Österreich weist ein strukturelles Defizit in der Technologischen Zahlungsbilanz auf. Die österreichische Deckungsquote war 1994 die drittniedrigste unter 16 erfaßten OECD-Länder. Im Jahr 1995 lag die Deckungsquote bei $25 \%$.

Nur wenige Länder sind Netto-Technologieexporteure. Die absolut höchsten Überschüsse weisen (nach wie vor mit großem Abstand) die USA aus, gefolgt von Japan und Großbritannien. Aber auch einige kleine entwickelte Volkswirtschaften erzielen Überschüsse im immateriellen internationalen Technologiehandel oder zumindest eine hohe Deckungsquote. Zu diesen Ländern zählen Schweden, die Niederlande und Belgien mit Deckungsquoten (1994 bzw. letztverfügbares Jahr) von 889\% (singulär hohe Einnahmen 1993), 101\% bzw. 94\%. Für die Schweiz liegen keine Angaben vor. In scharfem Kontrast dazu wies Österreich 1994 mit 29\% die - mit Ausnahme Spaniens und bemer- 
kenswerterweise auch Finnlands - geringste Deckungsquote unter 16 erfaßten OECDLändern auf (siehe Übersicht 3.3.1). Ein Defizit in der Technologischen Zahlungsbilanz kann (unter anderem) einerseits als Zeichen für technologische Rückständigkeit, andererseits jedoch als Kennzeichen von Ländern in einem technologischen Aufholprozeß interpretiert werden. Im zuletzt genanntem Fall kann jedoch erwartet werden, daß sich die Deckungsquote im Zeitablauf allmählich erhöht.

\subsection{Internationale Produktivitätseffekte von Forschung und Entwicklung}

Über Umfang und Rolle internationaler F\&E-Spillovers, d. h. der Wirkungen einer Veränderung der F\&E-Investitionen im Ausland auf die Produktivitätsentwicklung in Österreich liegen derzeit - im Unterschied zu anderen OECD-Ländern - keine detaillierten empirischen Untersuchungen $\operatorname{vor}^{13}$ ). Es gibt aber Hinweise dafür, daß diese eine bedeutende Rolle spielen. Eine bekannte ökonometrische Studie ${ }^{14}$ ) über die Periode 19701990 kam zu dem Ergebnis, daß die Totale Faktorproduktivität (=Verhältnis des aggregierten Outputs zur gewichteten Summe des Arbeits- und Kapitaleinsatzes) in Österreich außerordentlich stark auf relative Veränderungen des F\&E-Kapitalstocks in Deutschland reagiert. Nur für einige wenige Länder wurden höhere Elastizitäten der Totalen Faktorproduktivität in Bezug auf den F\&E-Kapitalstock eines Partnerlandes geschätzt (Belgien in Bezug auf den deutschen F\&E-Kapitalstock, Kanada, Israel, Irland, Belgien, Niederlande und Norwegen gegenüber jenem der USA).

${ }^{13}$ ) Eine empirische Untersuchung für Österreich wird im Rahmen des Programms tip durchgeführt.

${ }^{14}$ ) Coe - Helpman (1995). 


\section{Technologiediffusion im internationalen Vergleich}

Unter der Diffusion einer Technologie versteht man den Prozeß der Verbreitung einer Innovation vom erstmaligen Einsatz durch den Innovator bis hin zur weitverbrei teten, allgemeinen Anwendung. In den achtziger Jahren änderte sich die Auffassung von technologischem Wandel dahingehend, daß Technologiediffusion nicht einfach als linearer Transfer technischer Infrastruktur und zugehörigen Know-hows zu verstehen ist. Vielmehr resultiert die Diffusion von Innovationen aus dem komplexen Zusammenwirken von Forschern, Vermittlern und Anwendern. Im besonderen wurde auf der Unternehmensebene eine Reihe von Voraussetzungen identifiziert, welche die Adoptionsfähigkeit von Unternehmen für Innovationen nach haltig gewährleisten.

Aus diesem Grund legt die Innovationspolitik heute in vielen OECD-Staaten besonderen Wert auf die Beseitigung von Adoptionshemmnissen auf Unternehmensseite, meist mit einem Schwerpunkt bei kleinen und mittleren Unternehmen. In der Vielfalt der bestehenden öffentlichen Fördermaßnahmen sind verschiedene Optionen zur Beeinflussung der Technologiediffusion erkennbar.

- Die erste Option beinhaltet die Förderung der Verbreitung spezieller Technologien.

- Die zweite Option zielt - technologie- und branchenübergreifend - auf eine generelle Verbesserung der Fähigkeit von Unternehmen zur Übernahme extern verfügbarer Technologien ab. Dabei bieten die Programme technische Hilfestellung, Zugang zu Information oder Unterstützung zur Netzwerkbildung an.

- Die dritte Option richtet sich auf die Fähigkeit der Unternehmen, selbst Innovationen durchzuführen (anstatt fremde Technologien zu übernehmen). Dies geschieht durch Angebot von Programmen auf den Gebieten Innovationsmanagement, strategische Innovationsplanung oder Erweiterung der Know-how-Basis.

Im folgenden wird die Diffusion in zwei Technologiebereichen, und zwar in der Informationsund Kommunikationstechnologie sowie in ausgewählten Technologien für eine nachhaltige Entwicklung dargestellt:

- Die Informations- und Kommunikationstechnologie durchdringt als generische Technologie zunehmend alle Bereiche der Wirtschaft von der Sachgüterproduktion bis hin zu Dienstleistungen, und das bereits weitgehend gesteuert durch Marktmechanismen.

- Die Technologien für eine nachhaltige Entwicklung (sustainable development) hingegen scheinen - trotz intensiver Diskussion auch auf internationaler Ebene - gerade in Zeiten schärferer internationaler Konkurrenz, hoher Arbeitslosigkeit und des Abbaus sozialer Sicherung in den Hintergrund zu treten. Aus diesem Grund werden, unter Hinweis auf die aktuellen Forschungsschwerpunkte der EU, zwei als nachhaltig eingestufte Technologien behandelt: Der Kombinierte Gütertransport aus dem Verkehrsbereich und die Kraft-Wärme-Kopplung aus dem Bereich der Energieerzeugung. 


\subsection{Informations- und Kommunikationstechnologie}

Das rapide Wachstum des Einsatzes von Informations- und Kommunikationstechnologien (IKT) in allen Bereichen des wirtschaftlichen und gesellschaftlichen Lebens führt zu strukturellen Veränderungen, die unter dem Titel Informationsgesellschaft diskutiert werden. Diese strukturellen Veränderungen basieren auf geänderten technologischen Rahmenbedingungen wie z. B. der digitalen Aufbereitung und Verarbeitung von Information sowie der Nutzung von (Tele-)Kommunikationsnetzen und -diensten für die Verteilung von und für den dezentralen Zugriff auf Information. Die entstehenden Informationsinfrastrukturen, bilden die Basis für zahlreiche Dienste und An wendungen, deren Nutzung bei der Erstellung von Gütern und Leistungen, der Durchführung von Transaktionen sowie beim Konsum von Informationsangeboten international an Bedeutung gewinnt.

Der europäische Markt für Informationstechnologie (IT) umfaßt etwa 28\% des Weltmarktes und büßt insgesamt leicht an Bedeutung ein. Innerhalb Europas verbreitert sich die Kluft zwischen IT-intensiven und IT-schwachen Ländern. Einzig Österreich konnte als IT-schwaches Land stark aufholen und-gemessen an den Ausgaben für IT bezogen auf das BIP - Anschluß an den westeuropäischen Durchschnitt finden.

Der Weltmarkt für Informations- und Kommunikationstechnologie erreichte im Jahr 1996 ein Volumen von 1,1 Billionen ECU. Im OECD-Raum, der 92\% des IKT-Weltmarktes umfaßt, betrug das Wachstum dieses Sektors im Zeitraum von 1987 bis 1994 durchschnittlich 9,6\% und lag damit deutlich über dem allgemeinen Wirtschaftswachstum von $6,9 \%{ }^{15}$ ).

Im globalen Vergleich stellen die USA den wichtigsten IKT-Markt dar, noch ausgeprägter ist ihre Dominanz im Sektor Informationstechnologie (IT, d. h. IKT ohne den Bereich der Kommunikationstechnologie), in dem die USA im Jahr 1996 bei steigender Tendenz 41,4\% des Weltmarktes einnahmen. Wie aus Übersicht 4.1.1 zu entnehmen ist, fällt zur Zeit der Anteil Europas an den Märkten für IT (1996: 28,3\%) und IKT (1996: 29,7\%) leicht, wobei die relativ stärkere Position Europas im Bereich der Kommunikationstechnologie bemerkenswert ist. Die japanischen Märkte nehmen 1996 bei leichten Rückgängen 16,8\% (IT) bzw. 14,9\% (IKT) ein. Die fernöstlichen Tiger staaten und die übrigen Länder nehmen stark an Bedeutung zu.

Obwohl der IT-Markt in Europa schneller wächst als andere Industriebereiche, bleibt dieses Wachstum deutlich hinter dem IT-Wachstum in den USA und in Japan zurück. Aber auch innerhalb Europas läßt sich eine breite Kluft zwischen IT-intensiven und IT-schwachen Ländern feststellen.

$\left.{ }^{15}\right)$ EITO (1997). 
Die USA geben traditionell weitaus am meisten für IT aus. Nach einem sehr kräftigen Wachstum in den letzten Jahren waren es im Jahr 1996 über 4\% des BIP. Aber auch Japan holte seit 1993 ge genüber Europa mit überdurchschnittlich hohen Wachstumsraten auf und gab 1996 bereits $2,7 \%$ 
Übersicht 4.1.1: IT- und IKT-Märkte nach Weltregionen 1995-1997

\begin{tabular}{|c|c|c|c|c|c|c|c|c|}
\hline & \multicolumn{4}{|c|}{ IT } & \multicolumn{4}{|c|}{ IKT } \\
\hline & $\begin{array}{c}1996 \\
\text { in Mrd. ECU }\end{array}$ & 1995 & $\begin{array}{l}1996 \\
\text { in \% }\end{array}$ & 1997 & $\begin{array}{c}1996 \\
\text { in Mrd. ECU }\end{array}$ & 1995 & $\begin{array}{l}1996 \\
\text { in \% }\end{array}$ & 1997 \\
\hline Europa & 154 & 29,4 & 28,3 & 27,5 & 328 & 30,3 & 29,7 & 29,3 \\
\hline USA & 225 & 41,0 & 41,4 & 41,8 & 381 & 34,5 & 34,6 & 34,7 \\
\hline Japan & 92 & 16,9 & 16,8 & 16,6 & 164 & 15,1 & 14,9 & 14,6 \\
\hline 4 Tiger & 16 & 2,7 & 2,9 & 3,0 & 44 & 3,9 & 4,0 & 4,0 \\
\hline Rest & 57 & 10,0 & 10,5 & 11,1 & 185 & 16,2 & 16,8 & 17,4 \\
\hline Gesamt & 544 & 100 & 100 & 100 & 1.102 & 100 & 100 & 100 \\
\hline
\end{tabular}

Q: EITO, Europa $=$ West und Osteuropa, 4 Tiger $=$ Hong Kong, Südkorea, Singapur, Taiwan, Rest $=$ Rest der Welt.

Abbildung 4.1.1: IT-Ausgaben in Prozent des BIP im internationalen Vergleich, 1993-1996

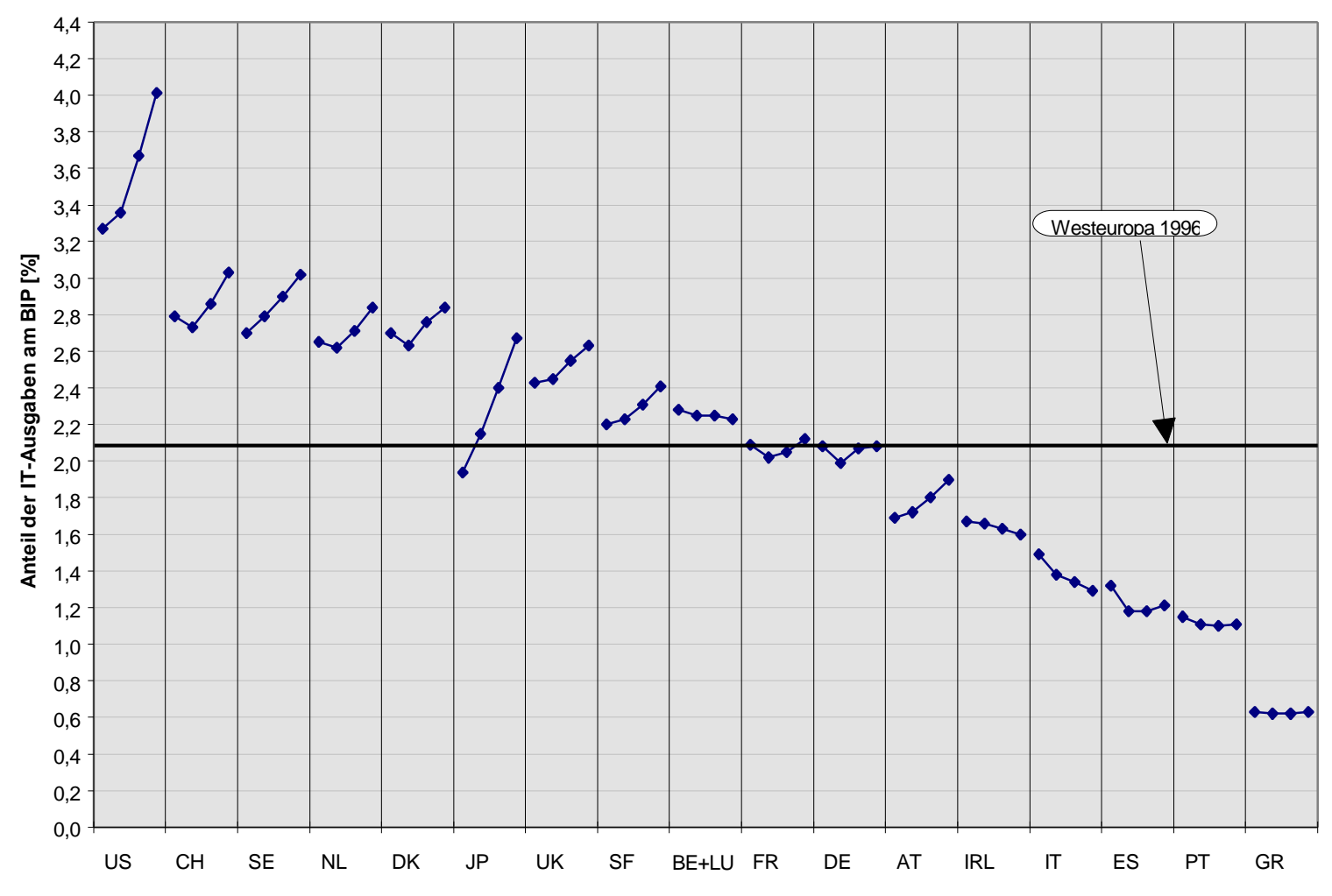

Q: EITO (1997). 
des BIP für IT aus. Dieser Wert liegt fast so hoch wie in den Vorreiterländern Europas, den skandinavischen Ländern, den Niederlanden und der Schweiz, deren Ausgaben nach moderatem Anstieg 1996 zwischen 2,8\% und 3\% des BIP liegen. Belgien, Frankreich und Deutschland liegen mit stagnierenden Anteilen am BIP etwa im westeuropäischen Durchschnitt von 2,1\%. Dagegen weisen die südeuropäischen Staaten auf niedrigem Niveau stagnierende oder, wie Italien, sogar deutlich fallende Anteile auf. Österreich hingegen findet nach einem bemerkenswerten Aufholprozeß mit zuletzt 1,9\% des BIP Anschluß an den westeuropäischen Durchschnitt (siehe Abbildung 4.1.1).

Der österreichische IKT-Markt wächst deutlich schneller als der im globalen Vergleich schwache EU-Durchschnitt. Dies ist hauptsächlich auf einen starken Aufholprozeß im Softwaresegment zurückzuführen.

Eine grobe Gliederung des IT-Sektors wird gemeinhin nach Hardware (vor allem PCs, Client-Server-Systeme), Software (PC-Anwendungen, Kommunikationssoftware) und Dienstleistungen vorgenommen. Daneben wird die Telekommunikation angeführt, was die zunehmende Verschmelzung von Informations- und Kommunikationstechnologien widerspiegelt, die vor allem durch die Verbreitung von Internet und Multimedia vorangetrieben wird. Die Telekommunikation ist gekennzeichnet durch die Entwicklung digitaler Übertragungstechniken, wie intelligente Netzwerke, Breitband-ISDN oder ATM, deren Ausbreitung in größerem Stil allerdings noch bevorsteht.

Der österreichische Markt für IKT nimmt 1996 mit 6.879 Mill. ECU etwa 2,3\% des EUMarktes ein. Aufgeschlüsselt auf die Segmente Hardware, Software und Dienstleistungen sind die österreichischen Märkte in Relation zu den jeweiligen EU-Märkten in Übersicht 4.1.2 dargestellt.

Die im globalen Vergleich schwachen Zuwachsraten des europäischen IT-Marktes werden in den meisten Segmenten von den österreichischen Märkten übertroffen, wenngleich nur wenig. Österreich hat leicht über dem EU-Schnitt liegende Wachstumsraten, die Dynamik läßt aber, dem europäischen Trend folgend, nach. Eine Ausnahme bilden die Dienstleistungen, deren Wachstum sich im EU-Durchschnitt beschleunigt, während es in Österreich weniger kräftig ist und nach den Prognosen des EITO in den nächsten Jahren sogar abnehmen wird.

Die Verteilung der IT-Ausgaben auf die Segmente Hardware, Software und Dienstleistungen ergibt im internationalen Vergleich folgendes Bild (siehe Abbildung 4.1.2): Auf den Bereich Hardware entfällt in fast allen Län dern der größte Anteil, Spitzenreiter sind Finnland und Spanien mit etwa 50\% des gesamten nationalen IT-Marktes. Ausnahmen bilden nur Frankreich und Italien, wo Dienstleistungen mit $42 \%$ bzw. $40 \%$ die bedeutendste Rolle spielen. Auch in Schweden (41\%) und Deutschland (35\%) kommt den Dienstleistungen erhebliche Bedeutung zu. Eine ausgeprägte Spezialisierung auf den Softwaremarkt weist Belgien mit über 30\% auf, wogegen in Schweden der Softwareanteil mit etwa $17 \%$ am niedrigsten ist. Für Österreich läßt sich im europäischen Vergleich der 
Marktsegmente mit 46\% Hardware, 31\% Dienstleistungen und 23\% Software keine besondere Ausprägung erkennen.

Übersicht 4.1.2: IKT-Marktsegmente in der EU und in Österreich

\begin{tabular}{lrrrrrrrrrr} 
& \multicolumn{1}{c}{ Volumen (in Mill. ECU) } & \multicolumn{7}{c}{ Wachstum (in \%) } \\
& 1994 & 1995 & 1996 & 1997 & 1998 & $95 / 96$ & $96 / 97$ & $97 / 98$ & $98 / 99$ \\
Computer Hardware & & & & & & & & & & \\
& & & & & & & & & & \\
& 1.287 & 1.274 & 61.813 & 65.908 & 70.260 & 9,1 & 6,1 & 6,6 & 6,6 & EU \\
Software & 24.200 & 26.507 & 28.866 & 31.521 & 34.492 & 9,5 & 8,9 & 9,2 & 9,4 & EU \\
& 578 & 664 & 754 & 843 & 927 & 14,8 & 13,6 & 11,8 & 10,0 & AT \\
Dienstleistungen & 43.066 & 45.267 & 47.729 & 50.366 & 53.599 & 5,1 & 5,4 & 5,5 & 6,4 & EU \\
& 1.051 & 1.105 & 1.163 & 1.213 & 1.262 & 5,1 & 5,3 & 4,3 & 4,0 & AT \\
IT gesamt & 120.662 & 130.048 & 138.408 & 147.796 & 158.350 & 7,8 & 6,4 & 6,8 & 7,1 & EU \\
& 2.916 & 3.173 & 3.429 & 3.686 & 3.952 & 8,8 & 8,1 & 7,5 & 7,2 & AT \\
Telekom gesamt & 133.466 & 145.733 & 157.302 & 168.788 & 180.420 & 9,2 & 7,9 & 7,3 & 6,9 & EU \\
& 2.934 & 3.175 & 3.450 & 3.743 & 4.075 & 8,2 & 8,7 & 8,5 & 8,9 & AT \\
IKT gesamt & 254.128 & 275.782 & 295.710 & 316.584 & 338.770 & 8,5 & 7,2 & 7,1 & 7,0 & EU \\
& 5.850 & 6.347 & 6.879 & 7.429 & 8.027 & 8,5 & 8,4 & 8,0 & 8,0 & AT
\end{tabular}

Q: EITO (1997); Die schattierten Felder bezeichnen jene Marktsegmente, in denen Österreich höhere Wachstumsraten aufweist als der EU-Durchschnitt. 
Abbildung 4.1.2: IT-Marktsegmente im internationalen Vergleich, 1995

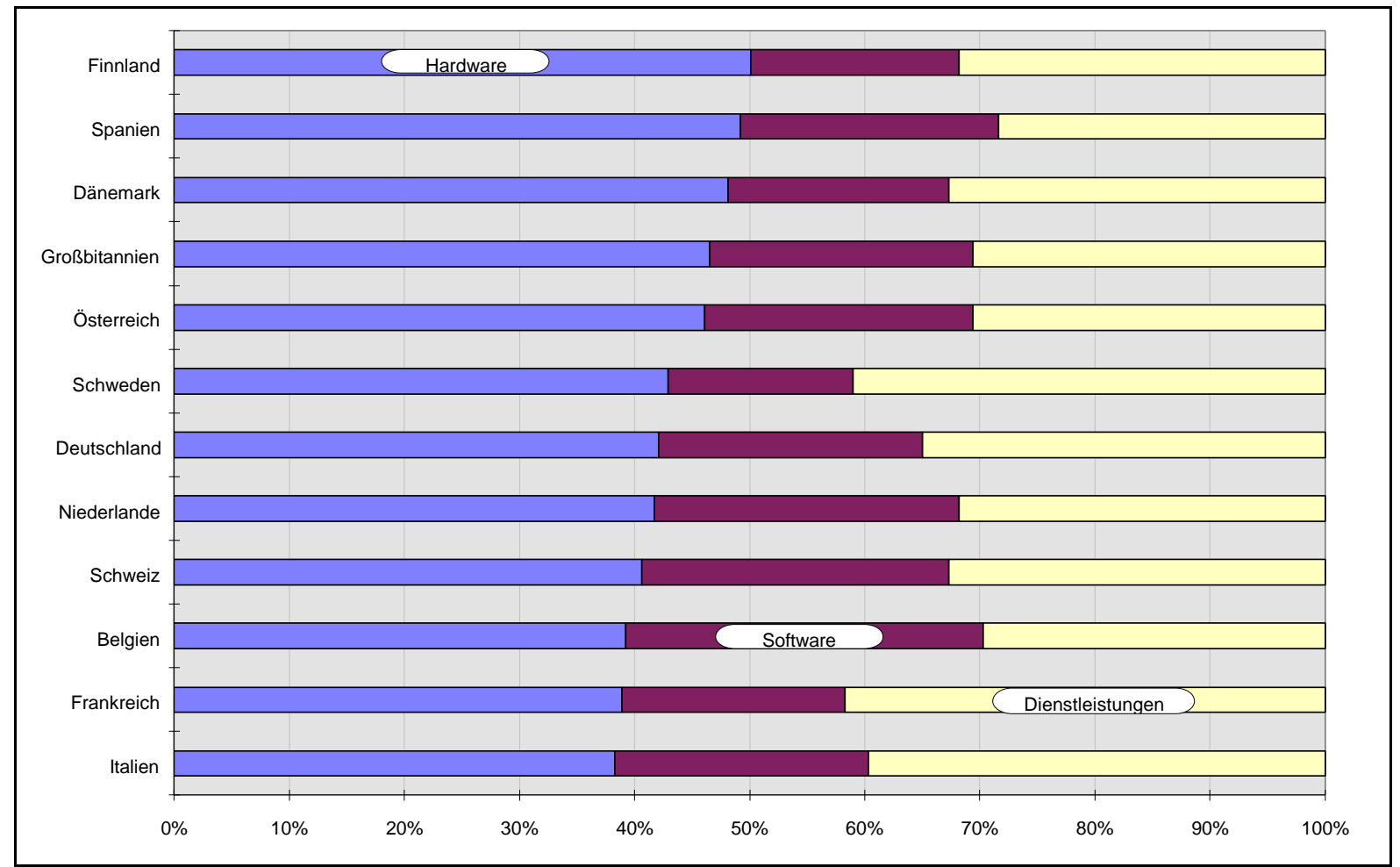

Q: OECD IT-Outlook.

In den USA entfielen 1994 auf Hardware und Datenkommunikationseinrichtungen etwa $44 \%$, etwa $20 \%$ auf Softwarepakete und $36 \%$ auf Dienstleistungen. Im selben Jahr kam das Hardwaresegment in Japan auf $56 \%$, und das Dienstleistungssegment auf $31 \%$. Softwarepakete nahmen in Japan nur 13\% des IT-Marktes ein.

Die IKT-Industrie ist eine Verbindung verschiedener Bereiche. Die Hardwareproduktion (Datenverarbeitungs- und Bürotechnik, Telekommunikation und elektronische Bauteile) zählen zum Industriesektor. Softwareproduktion, parallele und nachgelagerte Dienstleistungen und Dienste gehören zum Dienstleistungssektor. Wie sich in den letzten Jahren deutlich gezeigt hat, verlagert sich der Schwerpunkt zunehmend hin zur Produktion von Dienstleistungen und integrierten Lösungen. Die Gewinnspanne der Unternehmen hat sich immer mehr von der Hard ware- zur Softwareproduktion hin verschoben.

Die Produktion von Hardware ist in allen europäischen Ländern stark konzentriert (siehe Abbildung 4.1.3). Der Marktanteil der zehn größten (Top-10-)Unternehmen übersteigt überall 50\%, mit national unterschiedlichen Trends. In manchen Ländern, etwa in Schweden, ist zwischen 1994 und 1995 eine stark steigende Tendenz feststellbar. In Österreich nimmt die Konzentration leicht ab, von über 63\% im Jahr 1993 auf 58\% im Jahr 
1995. Die stärkste Abnahme verzeichnet Deutschland, wo die Konzentration im Hardwaresektor im Jahr 1995 auf 42\% gesunken ist.

Durchwegs deutlich weniger konzentriert (Top-10-Anteil unter 40\%) sind der Software- und Dienstleistungsbereich. Im Softwarebereich überwiegen die Konzentrationstendenzen, während der Dienstleistungssektor vor allem von kleinen und mittleren Unternehmen besetzt ist. In Österreich ist die Konzentration im Softwaresektor von 11\% (1993) auf über $25 \%$ (1995) gestiegen.

\subsection{Telekommunikationsinfrastruktur}

Der Verfügbarkeit und der Qualität der Telekommunikationsinfrastruktur kommt in mehrfacher Hinsicht hohe technologiepolitische Bedeutung zu. So wird etwa aufbauend auf ITAnwendungen durch die Nutzung von Telekommunikation die Grundlage für eine effiziente Abwicklung der Kommunikation in Zusammenhang mit wirtschaftlichen Aktivitäten gesetzt. Der Auf- und Ausbau der Infrastrukturen vollzieht sich unter den Bedingungen eines rasch voranschreitenden technologischen Wandels und bildet die Basis für Produktinnovationen und die rasche Diffusion neuer Telekommunikationsdienste und -anwendungen. Für die Modernisierung der Telekominfrastruktur selbst kommt neben den laufenden technologischen Entwicklungen die Änderung der Wettbewerbsregime auf den Märkten - Marktzutritt im Gefolge von Liberalisierungs bestrebungen und Zunahme der Wettbewerbsintensität zum Tragen. Hinsichtlich Ausbau und Modernisierung lassen sich trotz nationaler Unterschiede eine Reihe von gemeinsamen Entwicklungen feststellen. Das Festnetz für den Telefondienst bildet nach wie vor die wesentliche Basis der Telekommunikation, ist die Grundlage für den Zugang zum Teilnehmer sowie bestimmend für Investitionen und Erlöse der Öffentlichen Telekommunikationsnetzbetreiber (PTO, Public Telecommunications Operator). Die Liberalisierung des Marktzutritts im Bereich der Mobil kommunikation führt seit Anfang der neunziger Jahre zu einer rapiden Ausbreitung entsprechender Diensteangebote. Mit der steigenden Verbreitung des Internet entsteht eine Plattform für die standortübergreifende Datenkommunikation.

\subsubsection{Das digitalisierte Telefonnetz als Basisinfrastruktur}

Auch in Ländern mit hoher Marktdurchdringung ist trotz Abschwächung des Wachstumstrends ein signifikantes Teilnehmerwachstum beim Telefoniedienst festzustellen. Im Zeitraum von 1985 bis 1995 beispiels weise stieg die Marktdurchdringung (gemessen in Hauptanschlüssen pro 100 Einwohner) OECD-weit von 35,4\% auf 47,2\%. Selbst in Ländern, die bereits 1985 hohe Marktdurchdringungswerten hatten wie Schweden mit 62,8\% oder die Schweiz mit 50,2\% (zum Vergleich: Öster reich 36,1\%) wurden in diesem Zeitraum Steigerungen auf 68,1\% in Schweden bzw. 62,3\% in der Schweiz (zum Vergleich: Österreich 46,6\%) verzeichnet. Die Unterschiede in der Marktdurch dringung innerhalb der EU reichten 1995 von 36,1\% (Portugal) bis 68,1\% (Schweden); der Durchschnitt der 15 EUMitgliedstaaten lag mit 51,4\% nur geringfügig über dem Wert für Japan (48,8\%) jedoch deutlich unter dem Niveau in den USA (62,7\%) (siehe auch Abbil dung 4.2.1). 
Zwar ist in den Ländern mit vergleichsweise hoher Marktdurchdringung eine Wachstumsabschwächung beim Festnetztelefondienst erkennbar, für die meisten OECDLänder - darunter auch Österreich - sind beim bisher erreichten Niveaus dennoch Zuwächse zu erwarten. OECD-weit verringerte sich das durchschnittliche jährliche Teilnehmerwachstum im Zeitraum von 1990-1995 verglichen mit dem Zeitraum 1985-1990 lediglich von 3,97\% auf 3,93\%. Sättigungstendenzen führten in Schweden zu einer Abschwächung des Wachstums von $2,21 \%$ auf $0,56 \%$, in Österreich von 3,38\% auf 3,07\%.

OECD-weit ist eine leichte Sättigungstendenz im Wachstum des Telefonfestnetzes festzustellen. Die rasche Digitalisierung des Telefonnetzes ist eine Voraussetzung sowohl für Produktivitätssteigerungen als auch für die Erweiterung des

Serviceangebotes (z. B. ISDN).

Neben der Erschließung neuer Teilnehmer ist die Digitalisierung der Telefonnetze ein wichtiger Trend bei der Modernisierung der Netzinfrastrukturen, zumal dadurch beim Netzbetreiber eine Vereinfachung des Netzmanagements - und somit eine Erhöhung der Produktivität - sowie eine Erweiterung des Angebots neuer Dienste und Anwendungen erreicht werden kann. OECD-weit stieg der Anteil der an eine digitale Vermittlungsstelle angeschlossenen Teilnehmer zwischen 1990 und 1995 von rund $40 \%$ auf rund $83 \%$. Einen Digitalisierungsgrad von 100\% erreichten 1995 lediglich Frankreich, Island, Luxemburg und die Niederlande. In den Mitgliedsländern der EU lag der Digitalisierungsgrad nur in Griechenland (mit 35,3\%) deutlich niedriger als in den nächstfolgenden Ländern Deutschland und Spanien (beide 56\%), sodaß sich für den Durchschnitt der $15 \mathrm{EU}$ Mitgliedsländer mit $82,4 \%$ ein nur unwesentlich geringeres Niveau als in Japan $(90,4 \%)$ oder der USA (89,8\%) ergibt (siehe Abbildung 4.2.1). In Österreich führte eine Beschleunigung der Ausbauplanung der PTA zu einem Anstieg des Digitalisierungsgrades auf $72 \%$. 
Abbildung 4.1.3: Marktanteile der Top-10-Unternehmen in ihren IT-Sektoren, 1993-1995

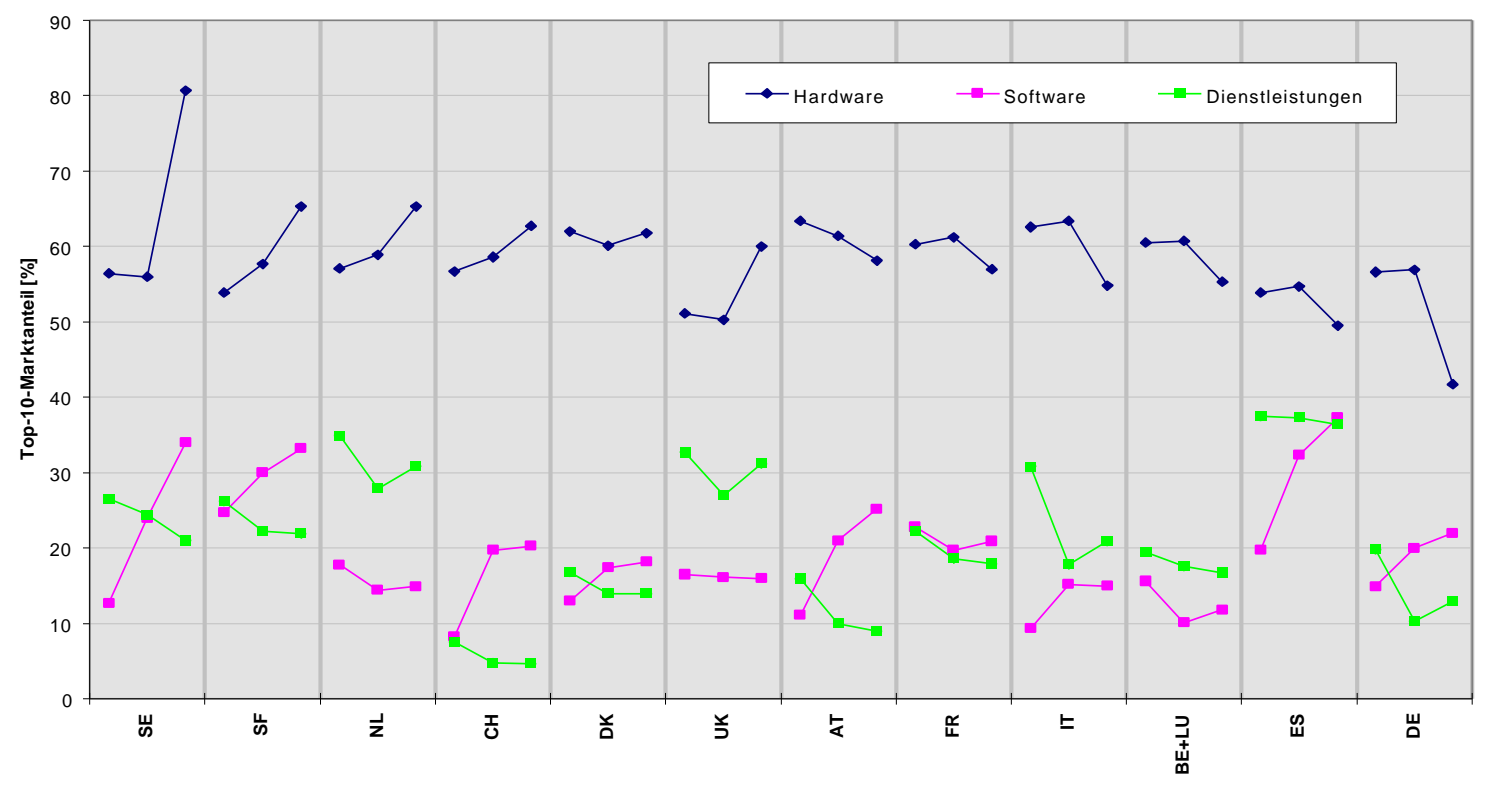

Q: EITO (1997).

Abbildung 4.2.1: Marktdurchdringung beim Telefondienst und Digitalisierung, 1995

•-Hauptanschlüsse pro 100 Einwohner (1995) $\rightarrow$ - Digitalisierungsgrad im Festnetz (1995)

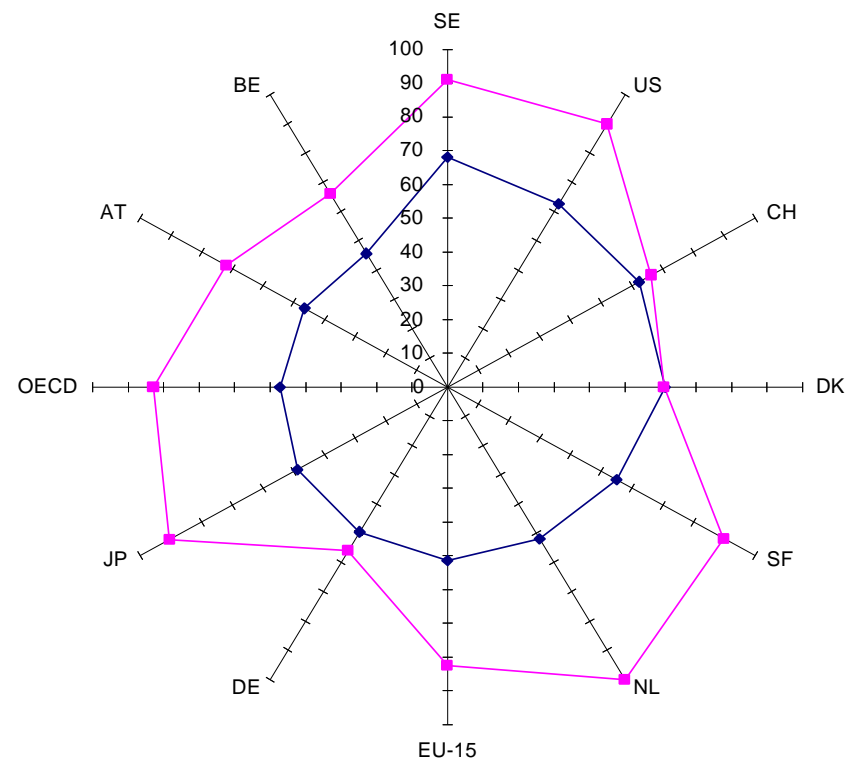

Q: OECD-ITU, OECD (1997). 
Der Digitalisierungsgrad selbst ist neben den Vermarktungsstrategien auch ein wichtiger Faktor für die Ausweitung des Angebots von ISDN (Integrated Services Digital Network). Beim ISDN-Angebot sind zwar in den letzten Jahren hohe Zuwachsraten erreicht worden, insgesamt liegt das Niveau allerdings - mit ausgeprägten nationalen Unterschieden der Nutzung - im Verhältnis zum Telefondienst niedrig. Führend in der Nutzung ist mit rund 960.000 der 1995 OECD-weit verfügbaren 2,5 Mill. ISDN-Anschlüsse die Bundesrepublik Deutschland, vor den USA, Japan und Großbritannien mit 507.000, 463.000 bzw. 260.000 Nutzern. Österreich lag mit rund 17.000 ISDN-Anschlüssen beträchtlich hinter der Schweiz (rund 70.000 Nutzer), aber nur unwesentlich hinter anderen vergleichbaren Ländern wie Schweden oder den Niederlanden (rund 20.000 bzw. 23.700 Nutzer).

\subsubsection{Mobil- und Datenkommunikation}

Das bei weitem stärkste Wachstum in der Nutzung moderner Telekommunikationsnetze entsteht derzeit im Bereich der Mobilkommunikation. Allein von 1990 bis 1995 wuchs die Anzahl der Mobiltelefonieteilnehmer innerhalb der OECD von rund 10,9 Mill. auf mehr als 74 Mill., was einer durchschnittlichen jährlichen Wachstumsrate von 46,6\% entspricht. Führend sind in diesem Bereich die skandinavischen Länder, die bereits gegen Mitte der achtziger Jahre analoge Mobiltelefonienetze aufgebaut haben und 1995 eine Marktdurchdringung (gemessen an Teilnehmern pro 100 Einwohner) von 22,8\% (Schweden), 22,5\% (Norwegen), 19,9\% (Finnland) und 15,7\% (Dänemark) erreichten. Für diese Länder ist auch abzusehen, daß im Jahr 1997 Marktdurchdringungen jenseits der 30\%-Marke erreicht werden. Wesentlich niedriger lagen 1995 die Durchdringungsraten in Ländern, die erst zu Beginn der neunziger Jahre mit einem Mobilkommunikations angebot für einen Massenmarkt auftraten wie z. B. die Schweiz (6,3\%), Österreich und Deutschland (je rund 4,6\%) oder die Niederlande (3,0\%). Der Durchschnitt der EU-Mitgliedstaaten lag 1995 mit $8,7 \%$ knapp vor Japan (8,2\%), aber deutlich hinter den USA (12,8\%) (siehe Abbildung 4.2.2). Die Marktdurchdringungsraten in den skandinavischen Ländern lassen darauf schließen, daß in den meisten OECD-Ländern eine Sättigung des Marktes für Mobiltelefonie auf absehbare Zeit nicht erreicht werden wird.

Mit hohen jährlichen Wachstumsraten (an die 50\%) entwickelte sich die Mobilkommunikation in den neunziger Jahren innerhalb der OECD überaus dynamisch. Eine Marktsättigung ist derzeit nicht in Sicht. Die Etablierung eines einheitlichen Digitalstandards und die Liberalisierung des Marktzuganges haben in Europa zu einer Vielzahl privater Netzanbieter geführt. Bereits 55\% der Mobiltelefonie wird in der EU über digitale Netze abgewickelt.

Zum rapiden Wachstum in der Mobilkommunikation hat insbesondere in Europa neben der technologischen Entwicklung (digitale, zellulare Systeme wie GSM und DCS-1800) eine Politik der Marktzutrittsliberalisierung wesentlich beigetragen. So wurden innerhalb der EU Maßnahmen verwirklicht, die zur Ausbildung eines ein heitlichen GSM-Standards und zur Erleichterung des Marktzutritts neuer Anbieter trotz zumindest im Festnetz 
marktdominierender PTOs beigetragen haben. Allein im Zeitraum von Oktober 1991 bis Oktober 1996 wurden innerhalb der EU 33 neue Mobiltelefonienetze - davon mehr als die Hälfte unter Beteiligung neuer Telekommunikationsanbieter - in Betrieb genommen; GSM und DCS-1800 sind in Europa die einzigen eingesetzten Technologien, sodaß bereits 1995 rund $55 \%$ der Mobiltelefonieteilnehmer in der EU ihre Kom munikation über digitale Netze abwickeln (siehe Abbildung 4.2.2). Die Wettbewerbsbedingungen unter denen Mobiltelefoniedienste nunmehr zumeist angeboten werden, führen im Gegensatz zur bislang meist vorherrschenden Monopolsituation sowohl zu niedrigeren Preisen als auch zu einer qualitativen Hebung des Angebots neuer mobiler Datendienste.

Ähnlich hohe Wachstumsraten wie die Mobiltelefonie verzeichnet das Internet, das die am weitesten verbreitete Plattform für Daten- und Textkommunikation von Computer zu Computer darstellt. Bereits etablierte Nutzungsmöglichkeiten des Internet wie Electronic Mail oder Zugang zu multimedialen Inhalten (etwa im World Wide Web) sowie künftige Anwendungen wie zum Beispiel Sprachtelefonie und Videoconferencing via Internet lassen auch weiterhin hohe Wachstumsraten erwarten.

Mit hohen Wachstumsraten verbreitet sich das Internet als Plattform für Datenkommunikation zwischen Computern. Ein Indikator für die Marktdurchdringung ist die Anzahl von Rechnern, die über das Internet erreichbar sind (Internet-Hosts). Finnland erreichte 1997 die international höchste Anzahl von Internet-Hosts in Relation zur Bevölkerung. Österreich liegt gemessen an diesem Indikator etwas unter den Durchschnittswerten der EU und der OECD jedoch deutlich vor Deutschland.

Einen Indikator für die derzeitige Entwicklung bildet die Anzahl der Internet-Hosts, also der über das Internet erreichbaren Computer. Innerhalb der OECD war im Zeitraum von Juli 1991 bis Jänner 1997 ein Anwachsen von rund 570.000 auf über 15 Mill. Internet-Hosts zu verzeichnen. Besonders hohe Ausprägungen sind in den skandinavischen Ländern sowie in den USA vorzufinden und spiegeln sich in der Markt durchdringung (siehe Abbildung 4.2.3) wider. Mit rund 555 Internet-Hosts pro 10.000 Einwohner zu Anfang 1997 erreicht Finnland die mit Abstand höchste Marktdurchdringung, liegt deutlich vor der USA (384) und vor den anderen skandinavischen Ländern (Norwegen, 394; Schweden, 264; Dänemark, 203) sowie der Schweiz (182). Die Marktdurchdringung in Österreich liegt gemessen an diesem Indikator mit 114 knapp unter den Durchschnittswerten der EU (124) und der OECD (149) jedoch deutlich vor Ländern wie Deutschland (88) und Belgien (63).

\subsection{Informationstechnologie in der Produktion: Industrieroboter}

Die industrielle Güterproduktion hat in den letzten Jahren einen umfassenden Wandel weg von starrer Massenproduktion hin zu wachsender Flexibilisierung vieler Produktions abläufe durchgemacht. Ausgelöst durch einen gestiegenen Bedarf nach Produktspezialitäten und raschen Produktinnovationen sind erhebliche Fortschritte bei der Umrüstbarkeit von Fertigungsanlagen und der Beschleunigung von Durchlaufzeiten erreicht worden. Unter 
dem Begriff Flexible Automation ist dieser umfassende Produktionsansatz zu einer Ganzheitstechnologie geworden. Die Kopplung aller informationserzeugenden und -verarbeitenden Teilschritte mit dem Ziel der gemeinsamen Nutzung einer einheitlichen Produkt- und Produktionsdatenbank ist das Konzept des Computer Integrated Manufacturing $\left.(\mathrm{CIM})^{16}\right)$. Eine wesentliche Basiskomponente darin bilden die Industrieroboter $(\mathrm{IR})^{17}$ ), die gleichzeitig in der Innovationsforschung als wichtiger In dikator dienen und als das Symbol für Flexible Automation schlechthin gelten.

Erste quantitative Modelle für technologische Innovationszyklen in Industriebranchen stammen aus den siebziger Jahren ${ }^{18}$ ). Diese Modelle beschreiben eine mehr oder weniger starre Automatisierung, die ausgehend von flexibler, nachfrageorientierter Produktion mit geringem Organisationsgrad in die Etablierung einer hochsystematischen Massenproduktion mündet. Seit der Verbreitung der Flexiblen Automation in den achtziger Jahren jedoch sind Flexibilität und Effizienz in der Pro duktion keine unvereinbaren Gegensätze mehr. Dies führt dazu, daß nicht mehr nur die reifen In dustrien automatisiert sind (starre Automation), sondern zunehmend die jungen, innovativen In dustriezweige, die sich in der Wachstumsphase befinden, in (flexible) Automatisie rung investieren. In einer kanadischen Untersuchung wurde darauf hingewiesen, daß die Hochtechnologie-Industrie den höchsten Grad an (flexibler) Automation aufweist ${ }^{19}$ ). Das technologische Niveau und das Marktwachstum sind danach statistisch die bedeutendsten Faktoren für die Automationsintensität ${ }^{20}$ ) eines Industriezweiges.

Die hohe globale Marktdynamik bei Industrierobotern wird von der überragenden Stellung Japans in der flexiblen Automation bestimmt, wo rund 60\% der weltweit eingesetzten Industrieroboter in Betrieb sind. Die hohen weltweiten Wachstumsraten von bis zu $20 \%$ in den achtziger Jahren scheinen zwar der Vergangenheit anzugehören, doch gehen Schätzungen für die nächsten Jahre wieder von einem stabilen Wachstum nahe $15 \%$ aus.

Nach dem ersten Industrieroboter-Patent im Jahr 1954 in den USA setzte die Verwendung von IR in der Industrie gegen Ende der sechziger Jahre ein. Seitdem erfuhren IR eine stürmische Marktentwicklung, die in bis zu 20\%igen Wachstumsraten in den achtziger Jahren gipfelte. In den neunziger Jahren drückte die Rezession in vielen Industrieländern die jährlichen Wachstumsraten auf durchschnittlich 6\%.

\footnotetext{
${ }^{16}$ ) WIFI (1989).

$\left.{ }^{17}\right)$ "Industrieroboter sind universell einsetzbare Bewegungsautomaten mit mehreren Achsen, deren Bewegungen hinsichtlich Bewegungsfolgen und Wegen bzw. Winkeln frei (d. h. ohne mechanischen Eingriff) programmierbar und gegebenenfalls sensorgeführt sind. Sie sind mit Greifern, Werkzeugen oder anderen Fertigungsmitteln ausrüstbar und können Handhabungs- und/oder Fertigungsaufgaben ausführen" (Zitat aus der Definition des VDI, Richtlinie 2860).

${ }^{18}$ ) Utterback - Abernathy (1975).

19) Martinez Sanchez (1995).

$\left.{ }^{20}\right)$ Anzahl der Industrieroboter bezogen auf Beschäftigte.
} 
Abbildung 4.2.2: Mobiltelefonie im internationalen Vergleich, 1995

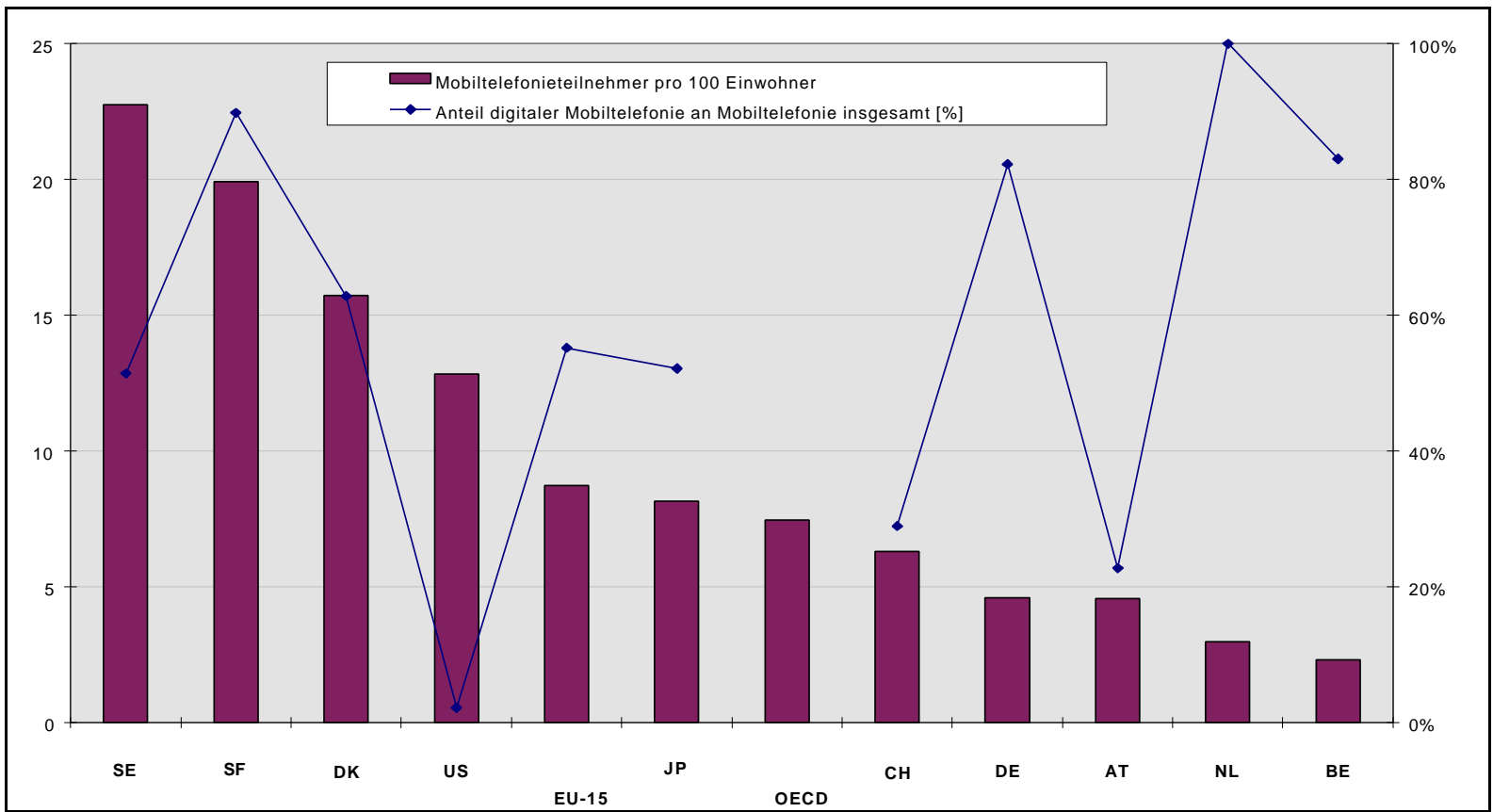

Q: OECD-ITU, OECD (1997).

Abbildung 4.2.3: Wachstum des Internet, 1994-1997

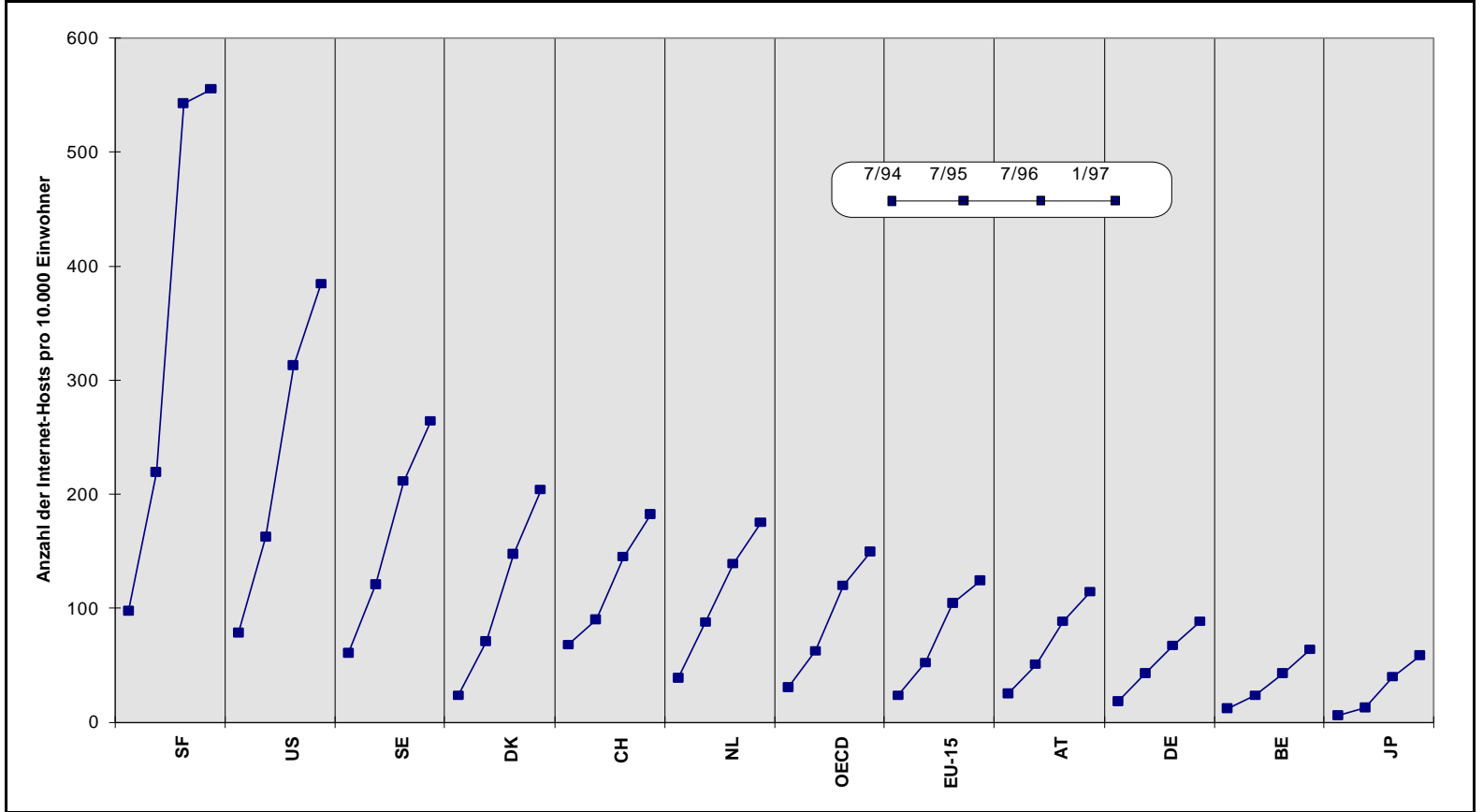

Q: OECD (1997), basierend auf Network Wizards [http://www.nw.com]. 
Bis 1995 wurden insgesamt weltweit etwa 790.000 Einheiten verkauft. Die Zahl der IR im Einsatz (operational stock, siehe Abbildung 4.3.1) ist etwas niedriger, da die Geräte nach einer gewissen Zeit wieder aus dem Betrieb genommen werden. Bei einer Einsatzdauer von 12 Jahren wird die Anzahl der 1995 in Betrieb befindlichen IR auf etwa 650.000 Einheiten geschätzt ${ }^{21}$ ).

Neben der Gesamtentwicklung ist aus Abbildung 4.3.1 die regionale Verteilung der IR ersichtlich. Im Jahr 1995 befanden sich knapp 60\% der weltweit betriebenen IR in Japan, das aufgrund dieser Dominanz die globale Marktdynamik bei Industrierobotern bestimmt. Starke Zuwächse wurden in den letzten Jahren in den ostasiatischen Ländern verzeichnet, besonders in Südkorea und Singapur, und dieser Trend wird sich den Prognosen zufolge fortsetzen. Die optimistischen Prognosen der UN/IFR für die nächsten Jahre gehen, mit regionalen Unterschieden, von jährlichen Wachstumsraten von etwa 15\% aus.

Der Automatisierungsgrad der Industrie ist weltweit deutlich im Steigen begriffen. In Japan ist er mindestens viermal so hoch wie in den wichtigsten Vergleichsländern. Mit Ausnahme von Japan und Norwegen hat auch das Wachstum des Automatisierungsgrades seit 1990 in allen untersuchten Ländern zugenommen. Besonders stark aufgeholt hat Korea, das 1995 an die weltweit zweite Stelle aufrückte.

Eine Kennzahl, die einen internationalen Vergleich des Automatisierungsgrades der Industrie ermöglicht, ist die Anzahl der Industrieroboter bezogen auf die Anzahl der Beschäftigten. Generell ist eine Vervielfachung dieser IR-Intensität im Zeitraum 1985 bis 1995 festzustellen (siehe Abbildung 4.3.2).

Die bei weitem höchste IR-Intensität weist 1995 Japan mit 251 IR pro 10.000 Beschäftigten auf, was annähernd der vierfachen Intensität der am stärksten automatisierten Vergleichsländer entspricht. Mit Ausnahme von Japan und Norwegen hat das Intensitätswachstum in allen betrachteten Ländern trotz der wirtschaftlichen Rezession nach 1990 weiter zugenommen. Dies ist ein Ausdruck der Tatsache, daß die Beschäftigung in der Industrie leicht abgenommen hat, bei gleich zeitig moderater Zunahme des IREinsatzes und somit der Produktivität. Für die nächsten Jahre wird eine weitere kräftige Zunahme der IR-Intensitäten erwartet.

Die traditionelle Vorreiterrolle in der flexiblen Automatisierung kommt der Automobilindustrie zu. Ihr Roboteranteil erreichte 1994 etwa in Italien und den USA fast den zehnfachen Wert der Industrie insgesamt.

Im internationalen Vergleich weist der Fahrzeugbau, insbesondere die Branche Motorfahrzeuge (Automobilindustrie), die höchsten Automatisierungsgrade auf, sowohl auf

$\left.{ }^{21}\right) \quad U N-I F R(1996)$ 
die Wertschöpfung als auch auf die Beschäftigung bezogen (siehe Übersicht 4.3.1). In den siebziger und achtziger Jahren hatte diese Branche die Vorreiterrolle bei der Verbreitung von IR gespielt, in den neunziger Jahren übernahm in einzel nen Ländern die Elektro- und Elektronikbranche die Vorreiterrolle. Dies gilt außer für das Muster beispiel Japan auch für Taiwan und für Länder mit relativ unbedeutendem Fahrzeugbau wie Österreich und Finnland.

Ein Einfluß der Roboterintensität auf die Arbeitsproduktivität ist nicht direkt gegeben. Dies ist zwar naheliegend, da die flexible Automation nicht in erster Linie auf Effi zienzsteigerung abzielt, sondern auf Flexibilität, Qualitätssteigerung und Arbeitsplatzverbesserung. Als Beispiel sei die besonders hoch automatisierte Branche Motorfahrzeuge dreier ausgewählter Länder angeführt (siehe Abbildung 4.3.3). Empirische Daten über die Entwicklung von Arbeitsproduktivität und Roboterintensität darin illustrieren, daß allgemein weder die Höhe der IR-Intensität als auch inre Steigerung im Zeitablauf (1975-1993) positiv auf die Arbeitsproduktivität wirken ${ }^{22}$ ).

Der Weltmarkt für Industrieroboter läßt starke Konzentrationstendenzen erkennen. Darüber hinaus ist die Nachfrage nach Industrierobotern überaus konjunkturreagibel.

In den achtziger Jahren war die Roboterindustrie von einer Vielzahl kleiner Unternehmen dominiert. Diese Zahl hat sich seitdem signifikant reduziert. Im Jahr 1995 setzten die drei größten Produzenten zusammen über 1,8 Mrd. \$ um, was einem Weltmarktanteil von 33\% entspricht. Noch 1990 kamen diese Firmen zusammengenommen auf einen Weltmarktanteil von nur 7\%.

Verglichen mit anderen Investitionsgütern sind die Absatzzahlen für Industrieroboter überaus konjunkturreagibel. Im Zug von Rezessionen fällt die Nachfrage nach IR und numerischen Maschinensteuerungen drastischer als für jedes andere Produkt, andererseits steigen auch die Wachstumsraten in Perioden wirtschaftlicher Erholung am schnellsten.

Übersicht 4.3.1: Roboteranteile industrieller Branchen bezogen auf ihre Wertschöpfungsbzw. Beschäftigungsanteile, 1994

\begin{tabular}{|c|c|c|c|c|c|c|c|c|c|c|c|c|c|c|c|}
\hline \multirow{2}{*}{\multicolumn{2}{|c|}{ ISIC rev.2 }} & \multicolumn{7}{|c|}{ Roboteranteil / Wertschöpfungsanteil } & \multicolumn{7}{|c|}{ Roboteranteil / Beschäftigungsanteil } \\
\hline & & JP & US & $\mathrm{DE}$ & $\mathrm{FR}$ & UK & IT & SE & JP & US & $\mathrm{DE}$ & FR & UK & IT & SE \\
\hline 3 & Industrie & 1,0 & 1,0 & 1,0 & 1,0 & 1,0 & 1,0 & 1,0 & 1,0 & 1,0 & 1,0 & 1,0 & 1,0 & 1,0 & 1,0 \\
\hline 38 & Engineering & 1,8 & & 1,4 & 2,0 & 1,7 & 2,5 & 1,8 & 1,7 & & 1,3 & 1,8 & 1,6 & 2,5 & 1,8 \\
\hline 381 & Eisen- und Metallwaren & 0,8 & & 1,0 & 0,2 & 1,4 & 2,1 & 2,6 & 0,6 & & 0,9 & 0,2 & 1,2 & 2,0 & 2,3 \\
\hline 382 & Maschinen- und Stahlbau & 0,9 & & 0,6 & 1,8 & 0,5 & 1,4 & 1,1 & 0,8 & & 0,5 & 1,4 & 0,4 & 1,5 & 1,0 \\
\hline 383 & Elektro- und Elektronik & 2,5 & & 0,5 & 0,5 & 0,7 & 1,3 & 1,1 & 2,2 & & 0,5 & 0,5 & 0,7 & 1,4 & 0,9 \\
\hline 384 & Fahrzeugbau & 2,6 & & 3,7 & 5,1 & 4,6 & 6,7 & 3,1 & 3,1 & & 3,7 & 4,4 & 4,9 & 6,7 & 3,1 \\
\hline
\end{tabular}

\footnotetext{
${ }^{22}$ ) Natürlich ist die Anzahl der produzierten Fahrzeuge als Maß für die Arbeitsproduktivität in der Automobilindustrie zunehmend weniger aussagekräftig, da (zumindest in den Industrieländern) auf gesättigten Märkten nur noch die Wertschöpfung erhöht wird, nicht aber die Anzahl der produzierten Fahrzeuge.
} 
Q: UN - IFR(1996).

\section{Abbildung 4.3.1: Anzahl der Industrieroboter ${ }^{1}$ ) nach Ländern, 1990-1995 und Prognose} 1996-1999

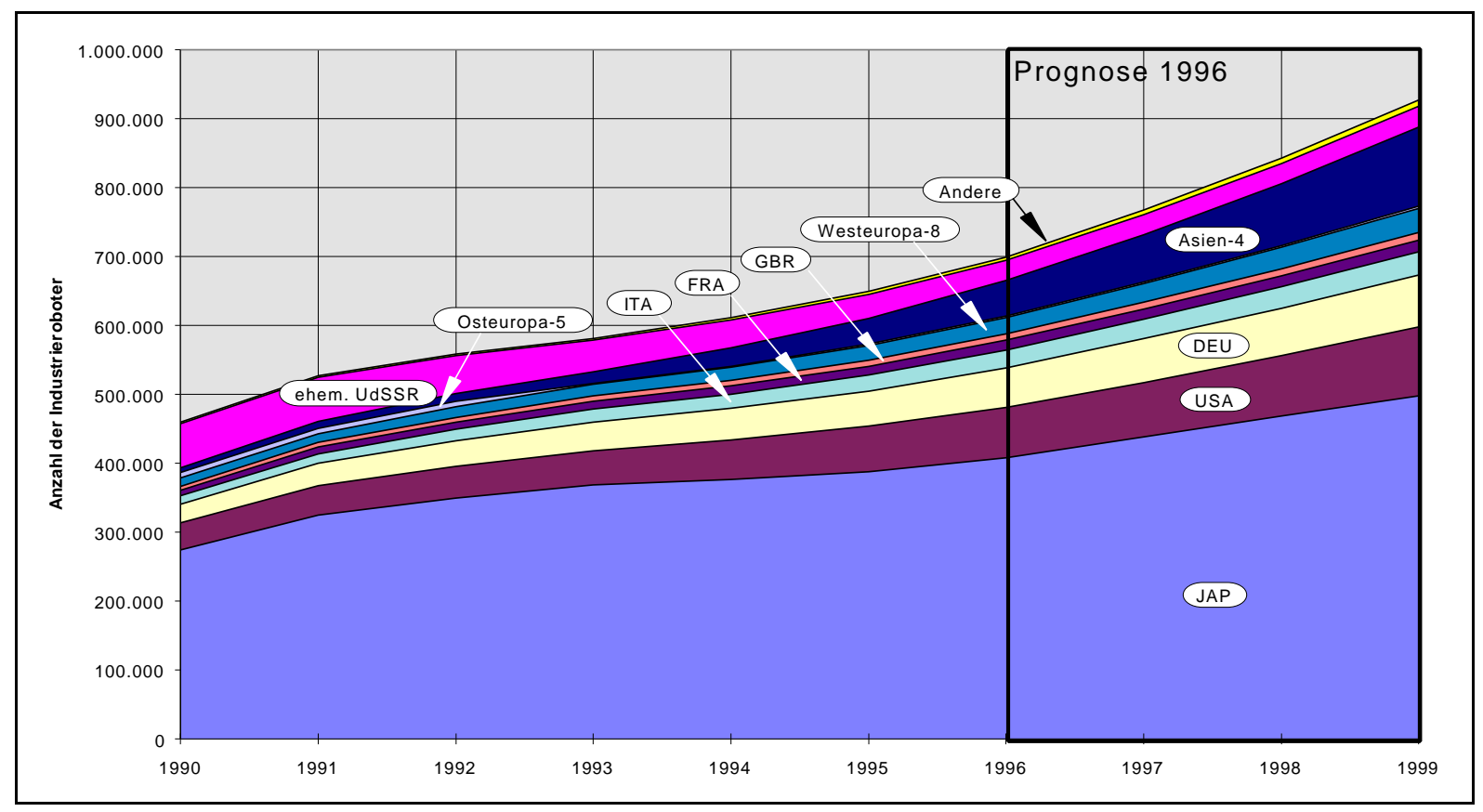

Q: UN - IFR (1996).

Legende: Asien-4: Australien, Singapur, Südkorea, Taiwan; Osteuropa-5: Polen, Slowakei, Slowenien, Tschechien, Ungarn; Westeuropa-8: Benelux, Dänemark, Finnland, Norwegen, Österreich, Schweden, Schweiz, Spanien.

$\left.{ }^{1}\right)$ Definition der IFR nach ISO 8373, operational stock. 
Abbildung 4.3.2: Industrieroboterintensität in den wichtigsten Industrieländern, 1985, 1990 und 1995

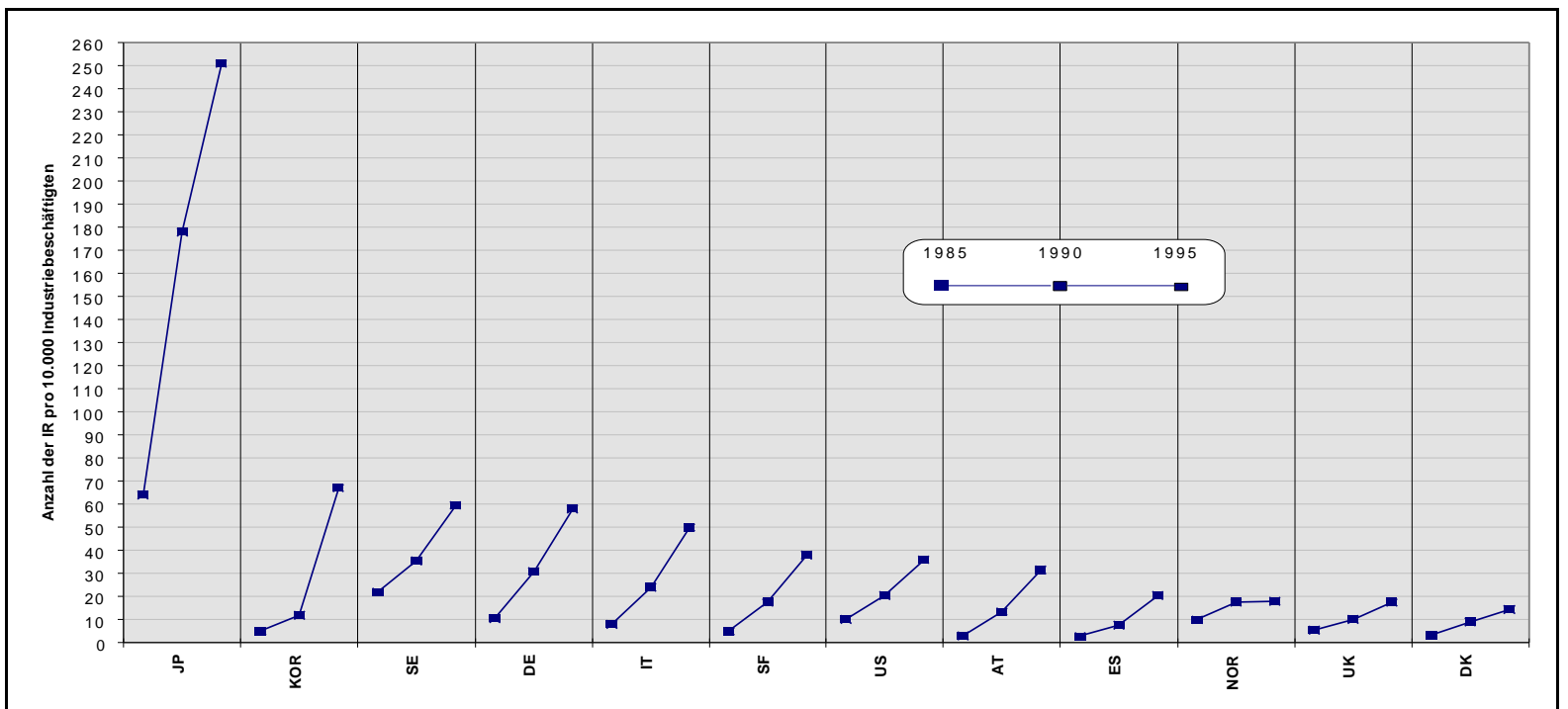

Q: UN - IFR (1996), eigene Berechnungen.

Abbildung 4.3.3: Arbeitsproduktivität verglichen mit Roboterintensität in der Automobilindustrie (ISIC rev.2 3843) in Italien, Japan und den USA, 1975-1993

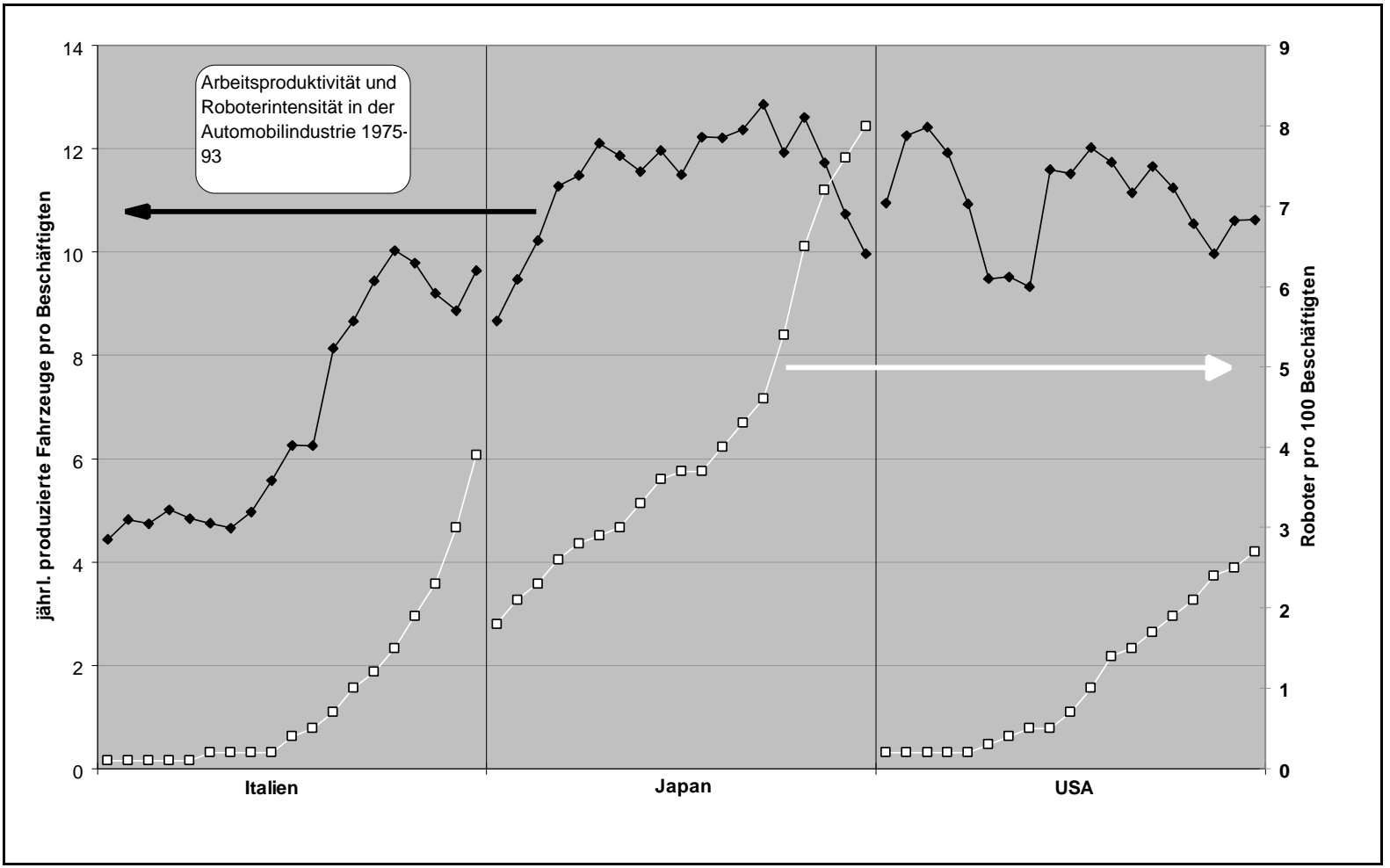

Q: UN - IFR (1996). 
Abbildung 4.3.4: Anzahl von Industrierobotern in Österreich, 1977-1995

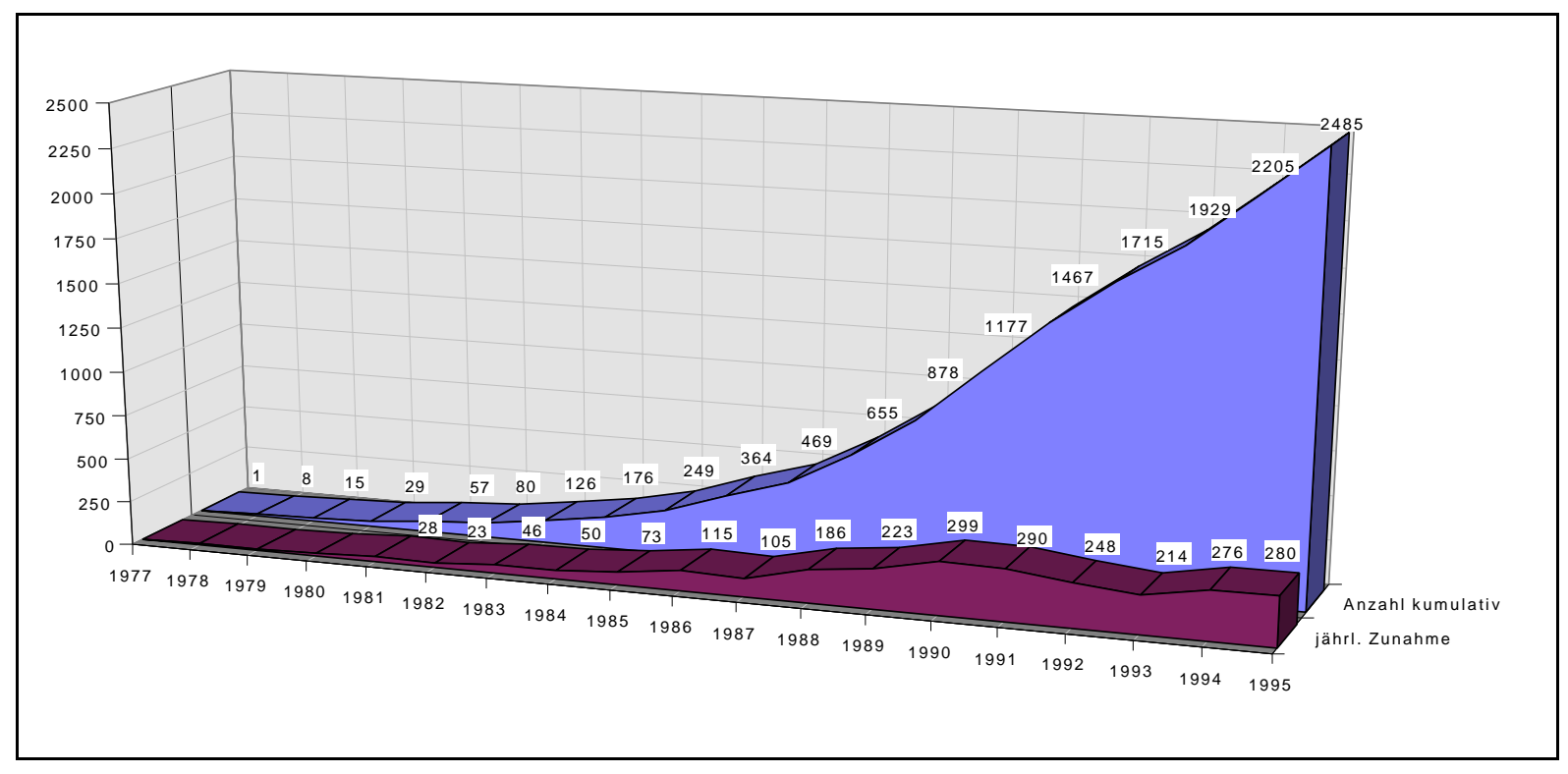

Q: WIFI (1995).

Die Automatisierung nahm in Österreich in den späten achtziger Jahren in allen Branchen stark zu. In den neunziger Jahren sind deutliche Verschiebungen bei IRNeuzugängen vom Fahrzeugbereich hin zu Elektro- und Elektronik sowie Maschinenund Stahlbau zu verzeichnen.

Die stärksten Zuwächse bei IR verzeichnet die Industrieroboterstatistik des WIFI ${ }^{23}$ ) Ende der achtziger, Anfang der neunziger Jahre. Im Jahr 1990 fanden die Investition en mit 299 Stück einen vorläufigen Höhepunkt (siehe Abbildung 4.3.4). Danach kam es zu einem deutlichen Rückgang. Im Jahr 1994 scheint sich wieder eine Umkehrung dieses Trends anzukündigen. Teilweise kann der Rückgang parallel zur internationalen Entwicklung der Rezession zugeschrieben werden.

Die österreichische Industrieroboterstatistik der Jahre 1986 bis 1994 zeigt starke Zuwächse in allen Branchen. Dabei sind jedoch deutliche Verschiebungen vom Fahrzeugbereich hin zur Elektro- und Elektronik- sowie zur Maschinen- und Stahlbauindustrie sowie eine Verbreitung auf mehr Branchen erkennbar. Noch 1986 waren der Fahrzeugbau mit 29\%, der Maschinen- und Stahlbau mit 25\%, sowie die Eisen- und Metallwarenindustrie mit 17\% des IR-Bestandes die drei wichtigsten Branchen. In den folgenden Jahren verschob sich der Schwerpunkt der Anwendung zur Elektro- und Elektronikindustrie, die 1994 mit einem Anteil von $25 \%$ die meisten IR einsetzte. Der Maschinen- und Stahlbau hielt den zweiten Rang, die Bedeutung des Fahrzeugbaus hingegen ging ständig zurück und lag 1994 mit

${ }^{23}$ ) Mooss (1995). 
etwa $15 \%$ des IR-Bestandes nur noch etwa gleichauf mit der Eisen- und Metallwarenerzeugung und dem Anteilsgewinner Chemische Industrie.

In Österreich verlagerte sich der Schwerpunkt der Automatisierung zu kleinen Unternehmen mit weniger als 200 Beschäftigten. Einen starken Anteilsrückgang verzeichneten hingegen die Firmen zwischen 500 und 1000 Beschäftigten.

Die Verteilung der Industrieroboter nach Unternehmensgrößenklassen zeigt von 1984 bis 1994 einen Anstieg der IR-Anteile in kleineren Unternehmen und eine Stagnation bzw. einen leichten Rückgang bei großen und mittleren Unternehmen. Auf Betriebe mit weniger als 200 Beschäftigten entfielen 1984 nur 27\% der IR, 1994 waren es bereits 35\%. Die stärksten Zuwächse verzeichneten Betriebe mit unter 50 Beschäftigten sowie Betriebe mit 101 bis 200 Beschäftigten. Den stärksten Anteilsrückgang verzeichnete die Größenklasse zwischen 501 und 1.000 Beschäftigten.

Ein gutes Drittel der Industrieroboter stammt aus heimischer Produktion, ein weiteres Drittel aus Deutschland. Die Angebotsseite ist hoch konzentriert, der schwedische Marktführer kommt auf 17\%, die Top-10 der Industrieroboter-Hersteller haben 56\% Marktanteil.

Die Herkunft der in Österreich in Betrieb genommenen Industrieroboter ist auf wenige Länder beschränkt. Die inländische Produktion ist dabei dominierend, 36\% kamen von österreichischen Produzenten, 34\% aus Deutschland und 17\% aus Schweden. Von den verbleibenden 13\% wurden mehr als drei Viertel aus Japan, der Schweiz und den USA eingeführt. Die Konzentration des IR-Marktes ist relativ hoch und vergleichbar mit jener des IT-Hardwaremarktes. Unter den 126 auf dem österreichischen Markt anbietenden Industrieroboterherstellern befinden sich nur 7 Großanbieter mit über 50 abgesetzten Stück (1977-1994). Die zehn wichtigsten Hersteller, 6 davon österreichische, halten zusammen $56 \%$ des Marktes, der Marktführer (zugleich das einzige schwedische Unternehmen) hält einen Anteil von etwa $17 \%$.

\subsection{Technologien für eine nachhaltige Entwicklung}

Unter dem Begriff nachhaltige Entwicklung (sustainable development) ist in den letzten Jahren eine ökologische und ökonomische Ziele einschließendes Konzept zum Leitbild der internationalen umwelt- und entwicklungspolitischen Diskussionen geworden. Auf EUEbene schlägt sich diese Sichtweise unter anderem im 5. Rahmenprogramm ${ }^{24}$ ) für Forschung, technologische Entwicklung und Demonstration nieder, das in den Jahren 1998 bis 2002 zur Umsetzung gelangen wird. Darin findet sich als eines von drei thematischen Programmen die Förderung von kompetitivem und nachhaltigem Wachstum ( Promoting

\footnotetext{
${ }^{24} \operatorname{COM}(97) 142$ fin.
} 
competitive and sustainable growth). Die beiden darin enthaltenen Ziele werden dabei in dem Sinn als komplementär betrachtet als es nur mit sauberen, intelligen ten Technologien gelingen wird, auf zukünftigen Märkten zu bestehen.

Wesentliche Schwerpunkte in den Aktionslinien des angesprochenen Programmes werden im Verkehrs- und im Energiebereich gesetzt. Nachhaltige Mobilität und Intermodalität zielen auf eine effiziente und ökologisch verträgliche Kombination der verschiedenen Verkehrsmodi, sowohl im Personen- als auch im Gütertransport ab. Das Ziel fortgeschrittener Energiesysteme und -dienstleistungen schließt die saubere Umwandlung und rationelle Verwendung der Energieträger mit ein. Diesen Zielsetzungen der F\&E-Politik der Europäischen Union entsprechend werden hier beispielhaft die Entwicklungen des Kombinierten Verkehrs und der Kraft-Wärme-Kopplung (Cogeneration) als wettbewerbsfähige und nachhaltige Technologien skizziert.

\subsubsection{Kombinierter Verkehr}

Ziel einer nachhaltigen Verkehrspolitik ist es, ein sicheres, effizientes und ökologisch verträgliches Verkehrssystem zu fördern, das sich aus einer optimalen Kombination von Straßen-, Schienen- und Wassertransport ergibt. Für die Verwirklichung einer solchen Nachhaltigkeit im europäischen Verkehrsnetz bietet sich mit dem Kombinierten Verkehr ein technisches und logistisches Instrument an, mit dessen Hilfe den ökonomischen, ökologischen und sozialen Problemen des Gütertransportes und im speziellen des Transitverkehres begegnet werden kann.

Als Kombinierten Verkehr (KV) versteht man üblicherweise die Verbindung des Gütertransportes per Lkw und per Bahn. Damit können die Vorteile der beiden Verkehrsmittel, flexible Verteilung in der Fläche auf der einen Seite und umweltfreundliche Überwindung großer Distanzen auf der anderen, genutzt werden. Die technischen und organisatorischen Schwachpunkte des KV liegen im Bereich der Schnittstellen zwischen den beiden Systemen, den Güterterminals. Grob unterscheidet man beim KV begleitete und unbegleitete Verkehre.

Erstere bezeichnen den Huckepackverkehr (Rollende Landstraße), bei dem der gesamte Lkw auf Niederflurwagen per Bahn transportiert wird. Die Vorteile der Rollenden Landstraße liegen im geringen logistischen Aufwand und in der flexiblen Einsetzbarkeit, da weder Infrastruktureinrichtungen wie etwa Güterterminals, noch spezielle Investitionen seitens der Spediteure notwendig sind. Nachteilig wirken sich die Ineffizienz des Transportes (hohe Totlast) und die Personalkosten dieser Technologie (Fahrer fährt mit), sowie Probleme bei der technischen Infrastruktur (Wagenmaterial, Tunnelgröße u. a.) aus. Zielgruppe der Rollenden Landstraße sind vor allem kleine Frächter und Unternehmen, besonders im Osten Europas, deren Alternative im Transport auf der Straße liegt.

Unbegleitete Verkehre (wie KV mit Containern, Wechselaufbauten, Sattelaufliegern u. a.) hingegen sprechen vor allem die großen Spediteure an. Sie bieten die Vorteile geringerer Personalkosten und des effizienten Transportes, stellen jedoch sehr anspruchsvolle 
logistische Systeme dar. Die Zukunft liegt Expertenmeinungen zufolge im liniengüterzugmäßigen Shuttleverkehr zwischen Güterterminals. Eine weitere Entwicklungschance bietet sich dem unbegleiteten Verkehr durch den Einsatz automatischer Ortungssysteme von einzelnen Verladeeinheiten mittels Telekommunikationstechnologie (Tracking und Tracing).

Die Rahmenbedingungen des allgemeinen Güterverkehrs in Europa führen zu einer konsequenten Verlagerung der Transportleistung von der Schiene auf die Straße.

Ein wesentliches Problem für den gesamten Kombinierten Verkehr sind die politischen Rahmenbedingungen, die zur bestehenden Wettbewerbsverzerrung zugunsten des LkwVerkehrs beitragen ${ }^{25}$ ). Werden die Lenk- und Ruhezeitregelungen für Lkw ignoriert, fällt der Kostenvergleich bei allen Technologien des KV und auf allen Transportdistanzen zugunsten der Straße aus. Bei deren Einhaltung wird der unbegleitete Verkehr ab rund $800 \mathrm{~km}$ Transportdistanz konkurrenzfähig. Nicht gedeckte externe Kosten im ökologischen Bereich, durch Lärmbelastung sowie durch Unfälle ergeben ebenfalls eine Begünstigung des LkwVerkehrs ${ }^{26}$ ).

Der allgemeine Güterverkehr in Europa zeigt neben einer stetigen Zunahme vor allem eine starke Expansion des Anteiles Straße zu Lasten der Anteile von Schiene und Wasserweg. Die gesamte Güterverkehrsleistung (gemessen in Tonnenkilometern, tkm) in der EU wies in den Jahren 1985-1990 eine mittlere jährliche Zunahme von etwa 3,5\% auf und erreichte 800 Mrd. tkm. Der Güterverkehr auf der Straße hat dabei von 59,4\% auf $65,4 \%$ der gesamten Transportleistung zugelegt. Der Transport auf Wasserwegen expandierte in diesem Zeitraum um 1,9\% pro Jahr ${ }^{27}$ ) Der Schienengütertransport in der EU verlor etwas an Bedeutung und hält bei knapp 22\% der gesamten Gütertransportleistung. Siehe auch Übersicht 4.4.1. Vor diesem Hintergrund sind die Grenzen des kombinierten Verkehrs in Europa zu sehen.

Der Kombinierte Verkehr in Europa konzentriert sich vor allem auf den ökologisch sensiblen Alpenraum, der eine geographische Barriere zwischen wirtschaftlich bedeutenden Regionen Europas darstellt.

Bezogen auf die gesamte europäische Gütertransportleistung weist der Kombinierte Verkehr einen annähernd konstanten Marktanteil von rund $4 \%$ auf. Eine bedeutendere

\footnotetext{
${ }^{25}$ ) So hat die seit Anfang 1995 eingehobene zeitabhängige Straßenverkehrsabgabe die anfallende Straßen benützungsgebühr für einen typischen Transport durch Österreich (Ost-West-Achse) per Lkw auf etwa ein Zehntel gesenkt.

${ }^{26}$ ) Die ungedeckten Infrastrukturkosten des Schienen- und des Straßengüterverkehrs (sie belaufen sich auf 270 bzw. $290 \mathrm{~S}$ je Tonnenkilometer) halten sich in etwa die Waage. Demgegenüber werden die ungedeckten externen Kosten des Lkw-Verkehrs im ökologischen Bereich deutlich höher eingeschätzt als jene des Bahnverkehrs (64-102 bzw. 166 S je Tonnenkilometer). Siehe Kageson (1993).

${ }^{27}$ ) Europäische Kommission (1996).
} 
Rolle spielt er vor allem in den Alpenländern mit ihrer spezifischen Transitproblematik, die durch die wirtschaftliche Bedeutung dieser Länder innerhalb Europas und durch die besondere ökologische Sensibilität der Alpenregion gekennzeichnet ist. In den letzten 25 Jahren hat sich der alpenquerende Gütertransport annähernd verdreifacht, das Transportvolumen erreichte 1994 rund 64 Mill. t, wovon 58\% auf den Lkw-Transport und $42 \%$ auf den Bahntransport entfielen. Länderweise verteilte sich dieser Verkehr zu $43 \%$ auf Österreich, zu 31\% auf Frankreich und zu 26\% auf die Schweiz. Die Bahngesell schaften haben den Kombinierten Verkehr allerdings in unterschiedlichem Maße zur Entlastung der betroffenen Straßentransitrouten eingesetzt.

Frankreich betreibt eine systematische Lkw-Güterverkehrspolitik. Bereits 1984 betrug der Bahnanteil am Alpentransit nur 25\%, bis zum Jahr 1994 ist er auf $15 \%$ gefallen. Im Gegensatz dazu wickelt die Schweiz als einziges Land einen Großteil des Transitvolumens auf der Schiene ab. Allerdings ist der Bahntransport unter Druck, sein Anteil ging im betrachteten Zeitraum von $94 \%$ auf $83 \%$ zurück. In Österreich als dem wichtigsten Alpentransitland wird wie in Frankreich der überwiegende Anteil des Transitaufkommens per Lkw transportiert. Österreich ist es hingegen gelungen, Transportleistung auf die Schiene zu verlagern und den Bahnanteil von 22\% im Jahr 1984 auf etwa 36\% im Jahr 1994 zu steigern ${ }^{28}$ ).

Die Abwicklung des kombinierten Verkehrs erfolgt in Europa über zwei internationale Gesellschaften, die Vereinigung der nationalen Kombigesellschaften (UIRR) und die Intercontainer-Interfrigo (ICF). Diese bilden das organisatorische Bindeglied zwischen den Bahngesellschaften und den Kunden. Die nationalen Kombigesellschaften sind im wesentlichen Zusammenschlüsse von Spediteuren und betreiben die Transporttechniken Wechselbehälter, Sattelauflieger und Rollende Landstraße. Die ICF befindet sich im Besitz von 27 europäischen Eisenbahngesellschaften und bietet Container (auch Kühlbehälter) und Wechselaufbauten an.

Die Entwicklung bei den Kombigesellschaften (UIRR) ist gekennzeichnet durch eine rasante Ausweitung des internationalen Verkehrs, die vor allem mit der Verwirklichung des europäischen Binnenmarktes zusammenhängt. Die nationalen Verkehre hingegen stagnierten. Entfiel 1981 noch mehr als die Hälfte der Transportleistung auf nationale Verkehre, so war es 1993 nur noch ein knappes Drittel. Deutschland, Italien, Frankreich, Österreich und die Schweiz stellen die im Verkehrsaufkommen wichtigsten nationalen UIRR-Mitgliedsgesellschaften. Bei der Intercontainer-Interfrigo (ICF) war in den Jahren 1991-1995 eine leichte Steigerung des Festlandverkehres zu verzeichnen. Schwerpunkte der ICF-Aktivitäten mit den bedeutendsten nationalen Beteiligungsgesell schaften sind die Länder Deutschland, Belgien, die Schweiz und Italien. Siehe auch Abbildung 4.4.1.

Das österreichische Güterverkehrsaufkommen im inländischen und grenzüberschreitenden Verkehr mit der EU erreichte 1994 etwa 208 Mill. t. Davon entfielen 52\% auf die Straße und 43\% auf die Bahn. Die Verkehrsentwicklung auf der Schiene war im Zeitraum von 1990 bis

${ }^{28}$ ) Molitor (1996). 
1995 im Schnitt durch eine niedrige jährliche Steigerungsrate von 1,9\% gekennzeichnet. Das gesamte Transportvolumen der Österreichischen Bundesbahnen erreichte im Jahr 1996 etwa 69,9 Mill. t. Die Aufschlüsselung nach Destinationen ergibt eine leicht sinkende Tendenz beim Inlandsverkehr (Sende- und Empfangsort in Österreich), auf inn entfiel 1996 nur noch rund ein Viertel des Gesamtvolumens. Die Aufwärtsentwicklung beim Schienengütertransport insgesamt ist hauptsächlich auf Durchfuhr und Einfuhr zurückzuführen, die in den betrachteten Jahren ein mittleres jährli ches Wachstum von 4,2\% bzw. 3,5\% verzeichneten ${ }^{29}$ ).

Im Kombinierten Verkehr in Österreich hingegen waren in den Jahren 1992 bis 1996 starke Zuwächse zu verzeichnen, die mittlere jährliche Wachstumsrate betrug 6,3\%. Verantwortlich dafür war vor allem der internationale Verkehr, und hier wiederum besonders die Einfuhr und der Transitverkehr. Beim Inlandsverkehr hingegen waren nur durchschnittliche Steigerungen zu verzeichnen, so daß sein Anteil knapp unter $10 \%$ des gesamten KV-Transportvolumens stagnierte. Die Exporte per Kombiniertem Verkehr dagegen waren anteilsmäßig etwas rückläufig. In Österreich lag 1995 der Anteil des Kombinierten Verkehrs mit knapp 10\% des gesamten Güterverkehrs mehr als doppelt so hoch wie im europäischen Durchschnitt. Prognosen für die nächsten Jahre sehen trotz der beim EU-Beitritt Österreichs verschlechterten Rahmenbedingungen für den Kombinierten Verkehr leichte Steigerungen voraus.

Ein österreichisches Spezifikum im Kombinierten Verkehr ist der hohe Anteil der Rollenden Landstraße, bedingt durch ein rasches Anwachsen des Transitverkehrs mit Osteuropa. Gerade die Rollende Landstraße hat jedoch durch den EU-Beitritt mit deutlich verschlechterten politischen Rahmenbedingungen zu kämpfen.

Kennzeichnend für die österreichische Situation bei der Verteilung auf unterschiedliche Technologien des Kombinierten Verkehrs ist der hohe Anteil des Huckepackverkehrs, der Rollenden Landstraße. Siehe auch Abbildung 4.4.2. Während im internationalen Vergleich 1995 nur 8\% auf der Rollenden Landstraße transportiert wurden, waren es in Österreich mehr als $40 \%$ des Kombinierten Verkehrs. Hauptgründe für diese Tatsache sind die sinkende Bedeutung des begleiteten Verkehres in Westeuropa und der rasche Anstieg des gesamten Transitverkehres mit den östlichen Nachbarländern Österreichs. Hier steigt man aufgrund fehlender Infrastruktur bei entsprechenden regulativen Maßnahmen leichter und schneller vom Lkw auf die Rollende Landstraße um als auf die logistisch aufwendigeren unbegleiteten Verkehre.

\subsubsection{Kraft-Wärme-Kopplung (Cogeneration)}

Ein Grundsatz der österreichischen Energiepolitik, nämlich ehestmöglich zu einer nachhaltigen Entwicklung im Energiebereich zu gelangen, beinhaltet die Sicherung von

${ }^{29)}$ ÖSTAT, ÖBB. 
Wirtschaftlichkeit und Umweltverträglichkeit der Energieversorgung ${ }^{30}$ ). Einen entscheidenden Faktor dabei stellt die Effizienz der Energieumwandlung dar, d. h. die Optimierung der Energiedienstleistung bei minimalem Primärenergieeinsatz. Unter der Kraft-Wärme-Kopplung (KWK) bzW. Cogeneration faßt man ver schiedene Technologien der kombinierten Erzeugung von Elektrizität und Wärme zusammen, wo bei die energetischen Verluste gegenüber konventionellen Umwandlungstechnologien wesentlich reduziert werden können ${ }^{31}$ ). Der Einsatz von Cogeneration-Anlagen reicht von der Raumheizung und Warmwasserbereitung (Niedertemperaturwärme) in Nah- und Fernwärmenetzen bis zur Erzeugung von Prozeßwärme (Hochtemperaturwärme) in der Industrie.

Ein Vorreiter bezüglich des Einsatzes der Kraft-Wärme-Kopplung (KWK) ist Dänemark. Aber auch in Österreich ist der KWK-Anteil am gesamten Kraftwerkspark mit 19\% der installierten Leistung im europäischen Vergleich relativ hoch.

An der Spitze beim Einsatz von KWK-Technologien liegt Dänemark auf, wo etwa $76 \%$ der gesamten installierten Kraftwerksleistung mit KWK ausgestattet sind (siehe Abbildung 4.4.3). Ebenfalls im oberen Bereich liegen Finnland und die Niederlande mit rund $30 \%$ KWK-Anteil, auch Österreich liegt mit 19\% im inter nationalen Vergleich relativ hoch. In Österreich waren im Jahr 1994 in den öffentlichen Elektrizi tätsversorgungsunternehmen 5.078,9 Megawatt Leistung in allen Wärmekraftwerken installiert ${ }^{32}$ ). Im Jahr 1995 waren in den öffentlichen Elektrizitätsversorgungsunter nehmen Österreichs 5.028 Megawatt Leistung in Wärmekraftwerken installiert ${ }^{33}$ ). Davon waren nach einer groben Schätzung nur knapp $20 \%$ mit KWK ausgestattet.

Die Primärenergieinputs erreichten dem gegenüber 1995 etwa 83.140 Terajoule (siehe Abbildung 4.4.4). Die Verteilung der Primärenergieinputs in öffentlichen KWK-Anlagen war in den Jahren davor durch einen kontinuierlich ansteigenden Gasanteil gekennzeichnet, der im Jahr 1994 bei über 54\% der gesamten Inputmenge lag. Der Anteil von Erdöl ist seit 1993 im Rückgang begriffen, ebenso der Anteil von Kohle. Der Einsatz von Biomasse in KWKAnlagen erreichte mit über $30 \%$ des gesamten Primärenergieeinsatzes einen vorläufigen Höhepunkt im Jahr 1991, danach war ein dramatischer Einbruch in der KWK-Erzeugung zu verzeichnen, der hauptsächlich auf die Biomasse zurückzufüh ren war. Erst 1995 erreichte der Biomasseeinsatz wieder das Niveau des Jahres 1987.

Im Jahr 1995 erreichte die Energieerzeugung in öffentlichen KWK-Anlagen 49.360 Terajoule, davon entfielen $59,6 \%$ auf die Stromproduktion und 40,4\% auf Fernwärme.

\footnotetext{
${ }^{30}$ ) Energiebericht 1996 der Österreichischen Bundesregierung.

${ }^{31}$ ) In Österreich betrug der Gesamtnutzungsgrad aller Cogeneration-Anlagen 1990 zusammengenommen rund $64 \%$, im Vergleich dazu erfolgte die Erzeugung von Strom in den reinen Kondensationskraftwerken ohne KraftWärme-Kopplung, d. h. ohne Nutzung der entstehenden Abwärme, mit nur 41\% Wirkungsgrad (Gilli et al., 1995).

$\left.{ }^{32}\right)$ ÖSTAT.

${ }^{33}$ ) ÖSTAT.
} 
Davor war im Jahr 1992 ein dramatischer Einbruch zu verzeichnen. Weiteres Potential für den Einsatz von KWK besteht vor allem in der Umrüstung von bestehenden kalorischen Kraftwerken sowie in der Errichtung von dezentralen Kleinkraftwerken für die Nahwärmeversorgung. Überdies läßt sich aus der seit 1991 ungebremsten Steigerung des Gesamtnutzungsgrades der öffentlichen KWK-Anlagen (siehe Abbildung 4.4.5) auf zusätzliches Verbesserungspotential schließen. Wird der damit eingeschlagene Innovationspfad weiter verfolgt, so wird die öffentliche KWK bis zum Jahr 2002 einen Gesamtnutzungsgrad von etwa $70 \%$ erreichen.

Potential für den verstärkten Einsatz der Kraft-Wärme-Kopplung in Österreich ist hauptsächlich im Bereich der öffentlichen kalorischen Stromerzeugung zu orten, und zwar sowohl die Effizienzsteigerung als auch die Verbreitung der Technologie betreffend.

Im Vergleich dazu umfaßten die industrieeigenen Wärmekraftwerke 1995 in Österreich eine installierte Leistung von 1.105,6 Megawatt, das entspricht etwa 18\% der gesamten kalorischen Kraftwerksleistung ${ }^{34}$ ). Die Eigenerzeugung spielt hauptsächlich in der Papierindustrie, aber auch in der Metallerzeugenden und der Chemischen Industrie eine Rolle, diese drei Branchen kommen für $88 \%$ der gesamten Eigenerzeugung in Österreich auf. Allerdings ist das Potential für KWK in der Industrie weitgehend ausgeschöpft. So stammten schon 1990 von den 66.400 Terajoule Gesamtenergie, die in IndustrieEigenanlagen erzeugt wurden, bereits 99\% aus KWK-Anlagen. Der Gesamtnutzungsgrad aller industrieeigenen Wärmekraftwerke betrug dabei $70,3 \%{ }^{35}$ ).

Übersicht 4.4.1: Entwicklung des EU-Gütertransportes auf der Schiene, 1985-1993

\begin{tabular}{|c|c|c|c|c|c|c|c|c|c|}
\hline Aufkommen & 1985 & 1986 & 1987 & 1988 & 1989 & 1990 & 1991 & 1992 & 1993 \\
\hline Mill. tkm & 178.771 & 171.188 & 171.081 & 173.721 & 176.461 & 174.613 & 176.393 & 165.370 & 152.346 \\
\hline $\begin{array}{l}\text { Veränderung gegenüber } \\
\text { dem Vorjahr }\end{array}$ & & $-4,2 \%$ & $-0,1 \%$ & $1,5 \%$ & $1,6 \%$ & $-1,0 \%$ & $1,0 \%$ & $-6,2 \%$ & $-7,9 \%$ \\
\hline
\end{tabular}

\footnotetext{
$\left.{ }^{34}\right)$ ÖSTAT.

${ }^{35}$ ) Gilli et al. (1995).
} 
Abbildung 4.4.1: Verkehrsleistung (Tonnenkilometer) im Kombinierten Güterverkehr in Europa, 1981-1995

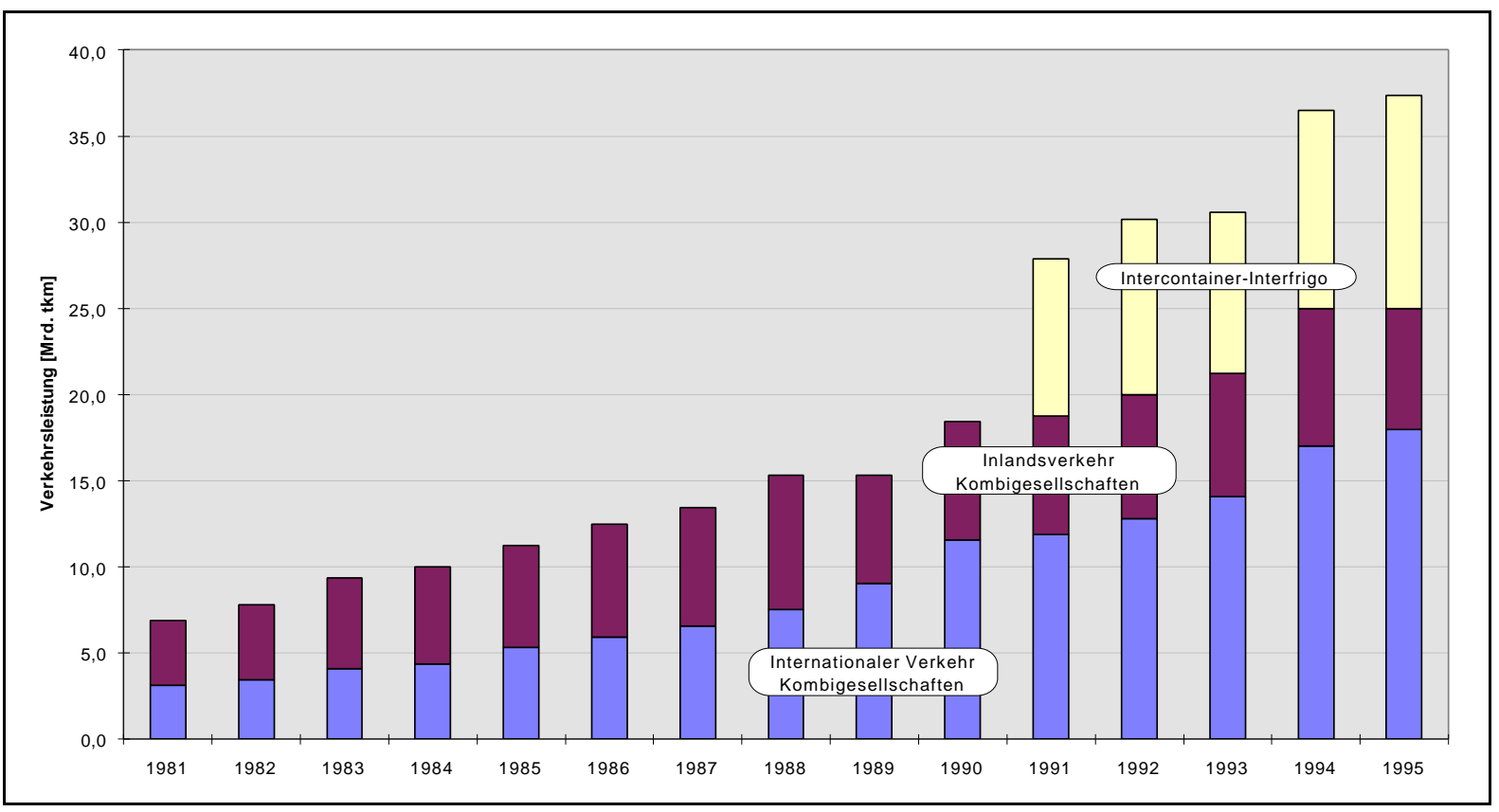

Q: UIRR, ICF (Daten vor 1991 nicht verfügbar).

Abbildung 4.4.2: Kombinierter Güterverkehr in Österreich nach Technologien, 1988-1995

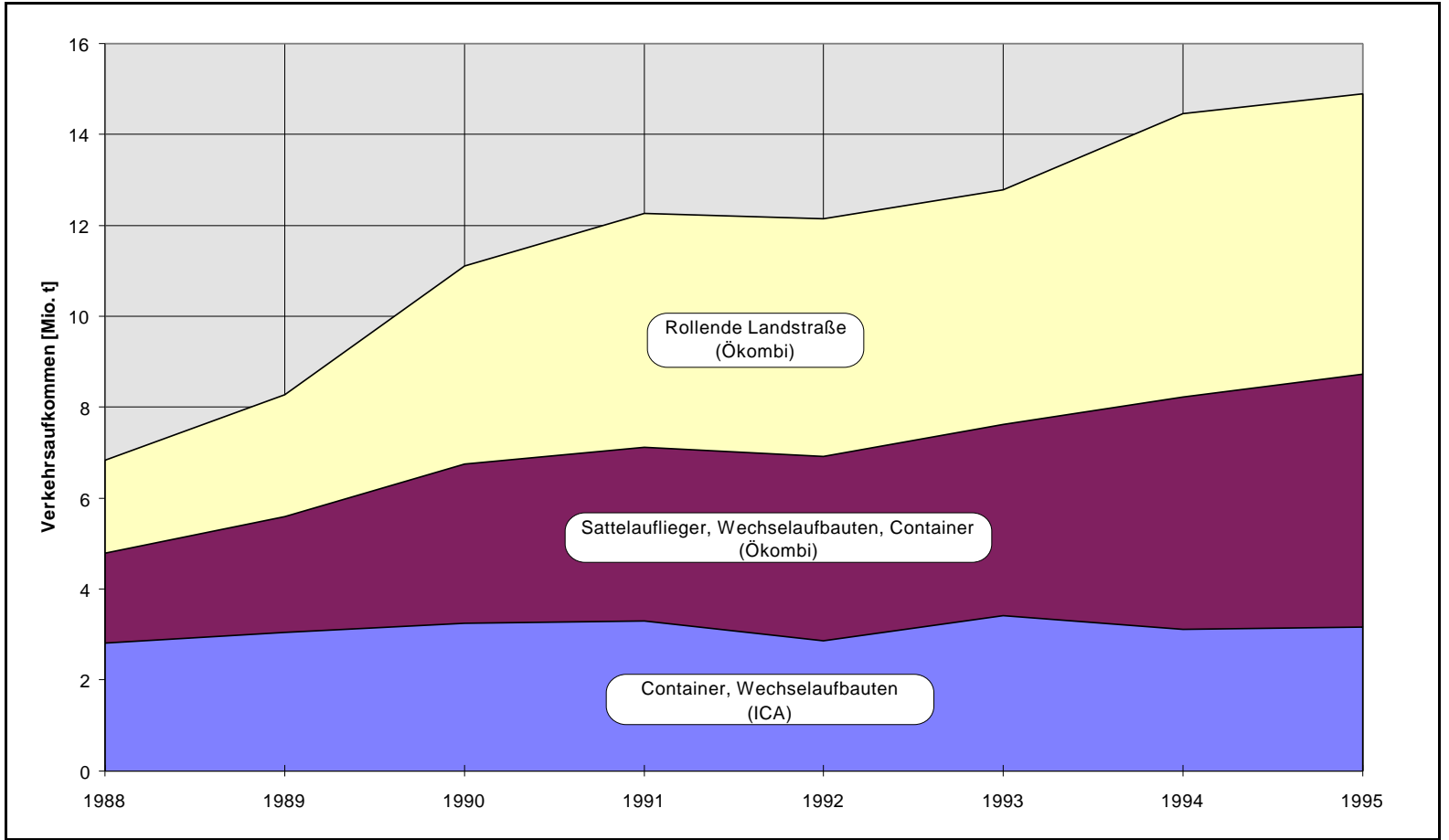

Q: ÖKOMBI. 
Abbildung 4.4.3: Installierte Kraftwerksleistung mit Kraft-Wärme-Kopplung im internationalen Vergleich, 1995

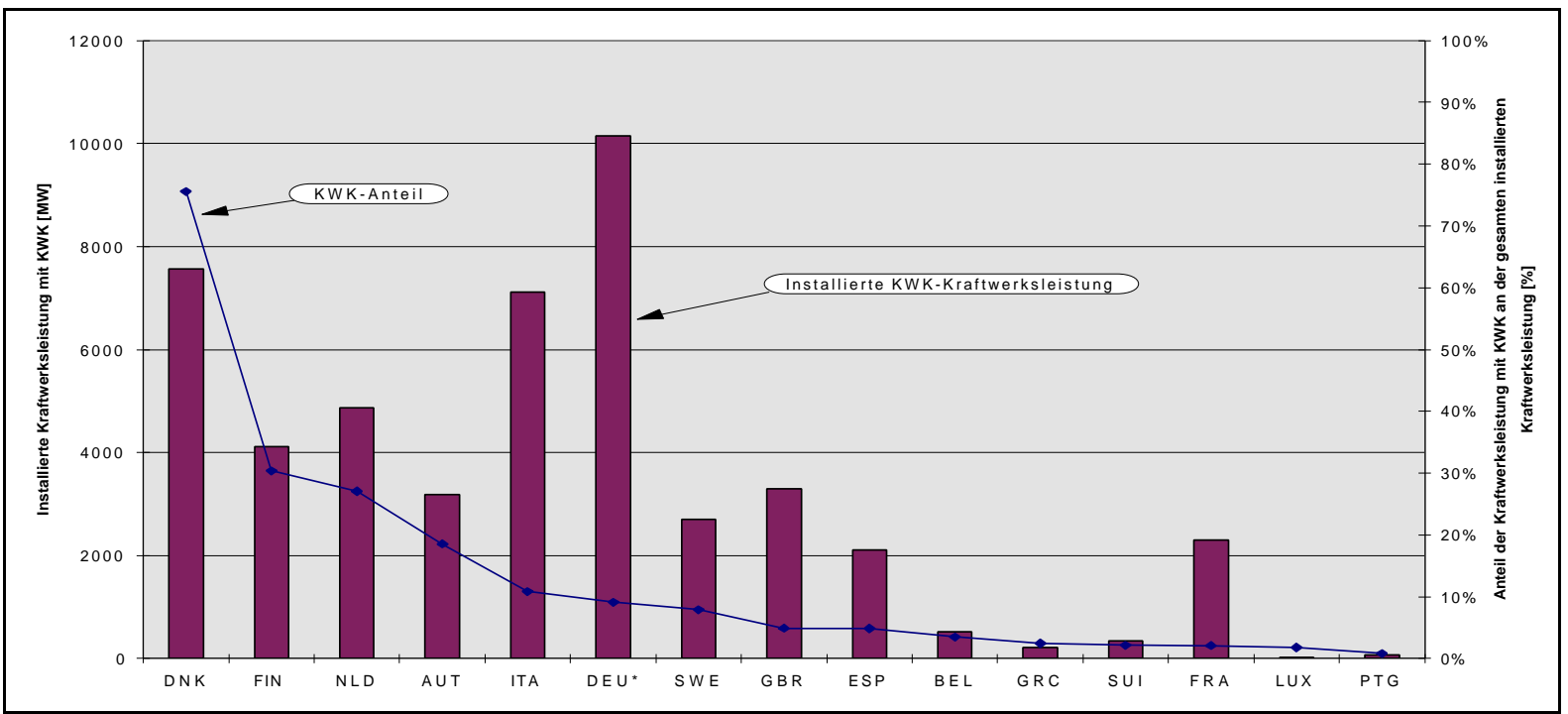

Q: Power in Europe, September 20, 1996, 233/8. * 1994.

Abbildung 4.4.4: Primärenergieinput der öffentlichen Cogeneration-Anlagen in Österreich (in Terajoule)

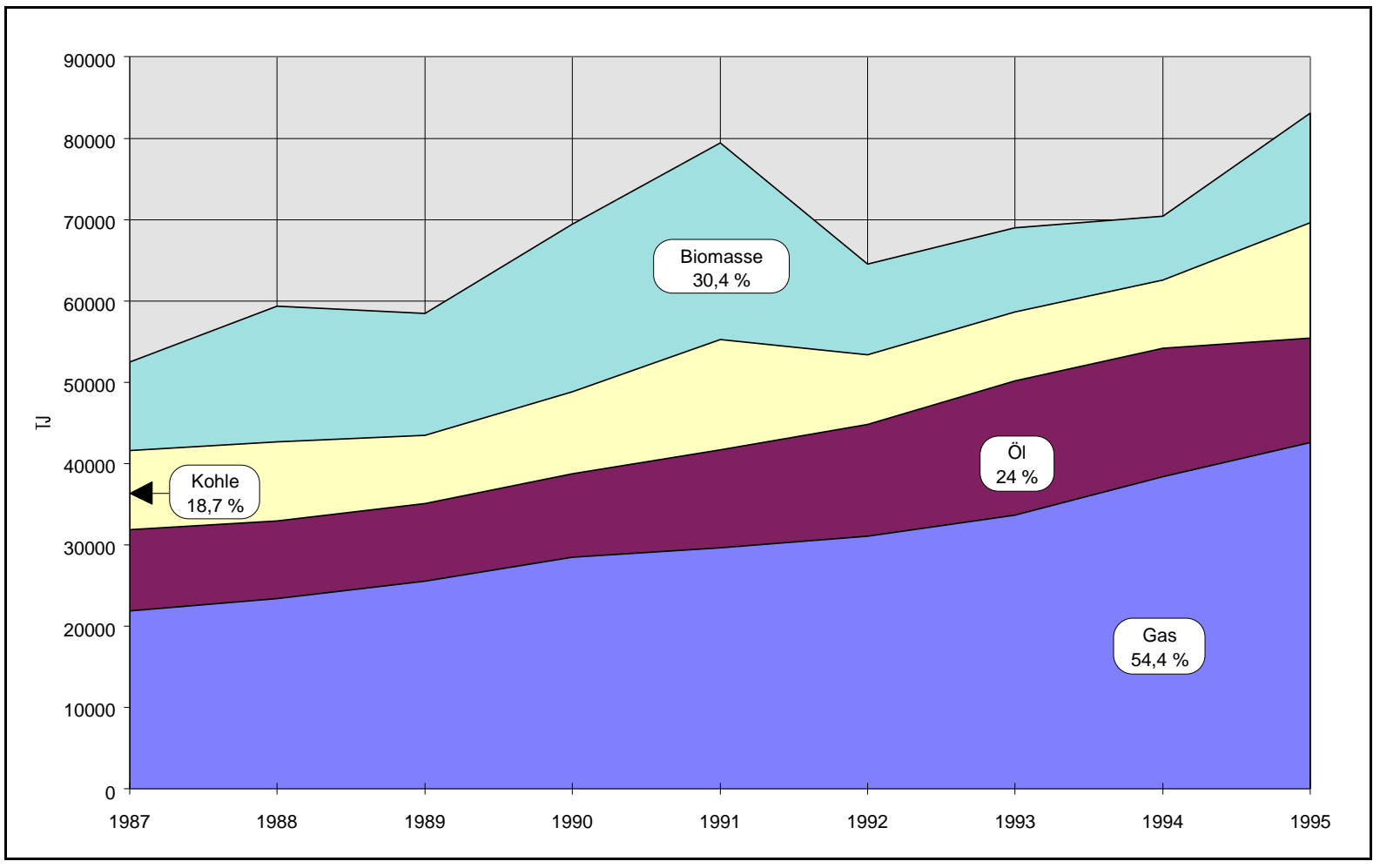

Q: E.V.A.; die Beschriftungen der Primärenergieträger befinden sich den Jahren ihres größten relativen Anteils. 
Abbildung 4.4.5: Energieerzeugung in öffentlichen Cogeneration-Anlagen in Österreich nach Endenergieträgern [Terajoule] und Gesamtnutzungsgrad, 1987-1995

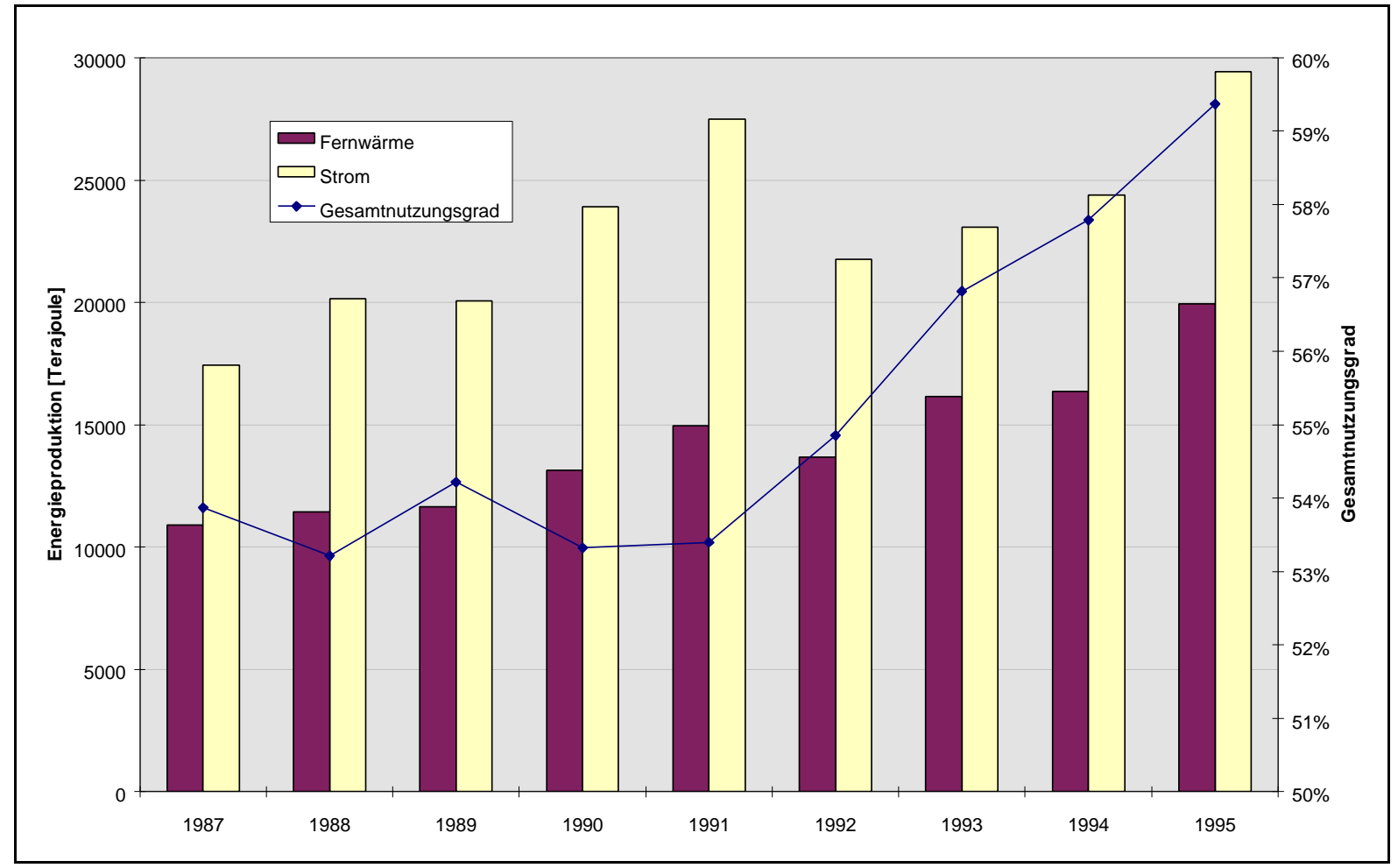

Q: E.V.A. 


\section{Spezialteil: Österreich und die Rahmenprogramme der EU}

Im Jahr 1996 wurde eine Evaluierung der österreichischen Beteiligung an gemeinschaftlichen Programmen im Bereich der Forschung und technologischen Entwicklung (FTE) durchgeführt ${ }^{36}$ ), bei der die Rahmenprogramme der Europäischen Union im Vordergrund standen. In dieser Evaluierung wurde das Ziel verfolgt, die Auswirkungen der gemeinschaftlichen FTE-Programme auf das österreichische Forschungs- und Innovationssystem zu untersuchen. Die maßgeblichen Fragen waren daher: Wie reagierte das österreichische Forschungs- und Innovationssystem auf die Möglichkeit der Teilnahme an europäischen FTE-Programmen? Welche Sektoren dieses österreichischen Forschungsund Innovationssystems reagierten und welche eher nicht? Wie veränderten sich die Verhaltensweisen und Prozesse auf der Ebene der teilnehmenden Organisationen? Welche strategischen Implikationen haben diese Veränderungen für die Teilnehmer selbst, für die Akteure der österreichischen Forschungs- und Technologiepolitik und für die Europäische Kommission?

Der vorliegende Spezialteil faßt die Hauptergebnisse der Evaluierungsstudie aus dem Jahr 1996 zusammen. Es ist zu beachten, daß sich diese auf den gesamten Beteiligungszeitraum (1984 bis 1996) bezieht. Diese Ergebnisse werden durch aktuelle Zahlen und Fakten über die österreichische Beteiligung am 4. Rahmenprogramm ergänzt, die eine positive Bilanz über den Verlauf der Integration Österreichs in das europäische Forschungssystem erlauben.

Teilnahme an gemeinschaftlichen FTE-Programmen: Die Entstehung eines europäischen Innovationssystems

Obwohl Österreich seit langem an internationalen FTE-Programmen teilgenommen hat, ist mit der Einführung der Rahmenprogramme, vor allem des dritten und vierten, eine neue Qualität in der internationalen Zusammenarbeit entstanden. Allerdings konnte Österreich seit der Einführung der Rahmenprogramme 1984 zunächst nur eingeschränkt an diesen teilnehmen - nur in bestimmten Programmen, projektweise und mit mindestens zwei ausländischen Partnern. Aus diesem Grund war eine in hohem Maß ungleiche Verteilung des Beteiligungsverhalten im Ersten und Zweiten Rahmenprogramm zu vermuten. Die empirische Analyse bestätigt in der Tat eine ungleiche Verteilung, sie zeigt aber auch, daß diese im Zuge des Dritten und Vierten Rahmenprogramms nach und nach verschwindet.

Eine detaillierte Analyse zeigt sogar, daß alle Mitgliedsländer eine in hohem Maß ähnliche Verteilung der Beteiligungsintensität verzeichnen: Langfristig betrachtet, beteiligen sich alle Länder - mit wenigen Ausnahmen - bei einem gegebenen Programm mit demselben Anteil ihrer Gesamtbeteiligung. Dies führt zum weitreichenden Schluß, daß die Rahmenprogramme als Kernstück europäischer Forschungs- und Technologiepolitik einen

${ }^{36}$ ) Ohler et al. (1997). 
hohen Steuerungseffekt (governance effect) ausüben, was als die Entstehung eines europäischen Innovationssystems interpretiert werden kann: Die Teilnahme an den Rahmenprogrammen reproduziert nicht existierende Forschungsprofile, im Gegenteil, die nationale Beteiligung wird von der Struktur der Rahmenprogramme bestimmt, nicht aber von der Spezialisierung des jeweiligen nationalen Forschungs- und Innovationssystems. Wie es scheint, verfügt jedes Land über eine Gruppe von Firmen und Instituten, die in der Lage und bereit sind, internationale Forschungskooperationen einzugehen, unabhängig von der Forschungs- und Industriestruktur des Landes.

\section{Beteiligungsintensität: Bevorzugung kleiner Länder}

Nehmen große Länder intensiver teil als kleine oder solche mit einer großen Zahl an Forschern gegenüber jenen mit einer geringen FTE-Quote? Welche Rolle spielt die nationale Finanzierung von FTE? Werden entwicklungsschwache Länder tatsächlich bevorzugt behandelt? Fragen wie diese sind eine unerschöpfliche Quelle an Spekulationen und Diskussionen. Die empirische Untersuchung konnte hier abhelfen, die diesbezüglichen Ergebnisse sind in hohem Maße überraschend: Die führende Gruppe bezüglich Intensität der Teilnahme sind Irland, Luxemburg, Dänemark, Bel gien, Niederlande, Griechenland und Portugal - ohne Ausnahmen kleine Länder. Im speziellen umfaßt diese Gruppe entwicklungsschwächere Länder wie Irland, Griechenland oder Portugal. Große und neu eingetretene Länder weisen dagegen eine mäßige Teilnahmeintensität auf. Öster reich war zum Zeitpunkt der Evaluierung das Land mit der geringsten Beteiligungsintensität (gemessen pro Einwohner bzw. pro Forscher). In entwicklungsschwachen und kleinen entwickelteren Ländern ist demnach die Leistungsfähigkeit des existierenden Forschungssystems im Sinne einer hohen Beteiligungsintensität am größten. Dies bedeutet, daß auch der Steuerungseffekt der Rahmenprogramme in diesen Ländern am größten ist (siehe Abbildung 5.1).

\section{Später Eintritt und Aufholprozeß}

Sind die später in die EU eingetretenen Länder in bezug auf ihre Beteiligung im Rückstand? In der Tat läßt sich ein derartiger Zusammenhang in bezug auf Schweden, Finnland und Österreich feststellen. Die Gründe für diesen Rückstand sind in der Vergangenheit zu suchen und rasch aufgezählt: (i) Ausschluß von einer Reihe von Programmen, (ii) Ausschluß von beratenden und entscheidungsfällenden Gremien und (iii) höhere Barrieren im Bilden von Projektkonsortien - Nicht-EU-Mitglieder mußten zwei - statt einen - Partner aus der EU haben. Allerdings haben sich die Verhältnisse im Zuge des Vierten Rahmenprogramms deutlich geändert: Die neuen Länder haben zwar noch nicht aufgeholt, sind aber dabei, dies zu tun: Österreich hat mit 50\% immerhin die höchste Wachstumsrate in der Beteiligung hinter Finnland mit 60\%. Diese hohen Wachstumsraten und können als Durchbruch in der Beteiligung im Beherrschen der Spielregeln verstanden werden (siehe Abbildung 5.2). 
Wichtigster Erfolgsfaktor: Zahl der eingereichten Projekte

Welche Faktoren erklären den Beteiligungserfolg? Ist es die Qualität der Anträge oder müssen die Antragsteller aus bestimmten Ländern kommen? Daten über die großen und einflußreichen Programme (BRITE-EURAM und ESPRIT) zeigen über die Teilnehmerländer hinweg eine extrem hohe Korrelation zwischen der Zahl der eingereichten und der Zahl der bewilligten Projekte. Dieser enge Zusammenhang führt zu einer sehr einfachen Schlußfolgerung: Um viele Projekte zu gewinnen, müssen viele Anträge gestellt werden. Die Erfolgsrate variiert zwar von Programm zu Programm, bleibt aber in nerhalb des Programms von Land zu Land gleich. Der Prozeß der Projektbewertung und -auswahl ist daher in hohem Maß konsistent, gelenkt von weithin akzeptierten Regeln und Prozeduren.

Diese Beobachtung läßt allerdings eine Frage unbeantwortet: Wenn die Qualität der Anträge das einzige Auswahlkriterium darstellt, warum kann jedes Land das gleich hohe Niveau an Qualität aufbringen, wo doch bekannt ist, daß unterschiedliche Länder unterschiedliche Spezialisierungsmuster und daher Kompetenzen aufweisen. Als Erklärung bietet sich an: Die Evaluatoren und die Kommission sind nicht abhängig von allen Anträgen, vielmehr können sie aus einem Fundus von 100 Projekten die besten 20 oder 30 auswählen und diese gibt es offenbar in jedem Land, was wiederum die Beobachtung bestätigt, daß das Beteiligungsverhalten relativ unabhängig von der nationalen Spezialisierung ist.

\section{Aufbau von Netzwerken und Partnerschaften}

Wer sind die Partner österreichischer Teilnehmer? Kommen sie aus Deutschland, dem wichtigsten Handelspartner, mit dem Österreich die Sprache teilt? Ist es der Klub der früheren EFTA-Länder oder hängen sich Österreicher an die angeblich begünstigten Länder wie Griechenland, Irland oder Portugal an? Die empirische Analyse liefert auch hier einige Klarstellungen: Österreich kooperiert am häufigsten mit den großen Ländern: Deutschland, Frankreich, Großbritannien und Italien, was wenig überrascht. Nachbarschaft und Intensität der Handelsbeziehungen spielen offenbar keine bedeutende Rolle. Gleichzeitig läßt sich zeigen, daß Österreich - was die Intensität der Kooperationsbeziehungen betrifft - am meisten mit kleinen Ländern zusammenarbeitet: Luxem burg, Irland, Dänemark, Finnland, Schweden, Belgien, Niederlande und Griechenland. Am intensivsten kooperieren allerdings Österreicher mit anderen Österreichern.

Eine besondere Stellung in Projektkonsortien nehmen die Koordinatoren ein. In innen werden gewöhnlich die treibenden Kräfte und die Entrepreneure innerhalb des Projektteams vermutet. Ein Blick auf die Ergebnisse der Evaluierungsstudie zeigt ein von diesem Stereotyp abweichendes Bild: Organisationen aus den Niederlanden (17\% aller Koordinatoren in Projekten mit österreichischer Beteiligung), Großbritannien (15\%) und Deutschland (13\%) hatten die größten Anteile. Die Erklärungen hierfür liegen weitgehend auf der Hand: Die Niederländer sind mehrsprachig und daher als Koordinatoren 
willkommen. Die Briten nehmen u. a. aufgrund der englischen Sprache bevorzugt die Rolle des Vis-à-vis gegenüber der Kommission ein, deutsche Partner sind aufgrund der gemeinsamen Sprache ebenfalls natürliche Kandidaten für die Rolle des Koordinators. Fügt man diese Argumente zusammen, so ergibt sich ein klarer Schluß: Sprache spielt eine nicht unerhebliche Rolle. Dies widerspricht zwar nicht der eingangs aufgestellten These vom Koordinator als Entrepreneur, differenziert aber und fügt diesen Charakteristika noch jene der Sprache und der administrativen Fähigkeiten hinzu. Box 3 enthält entsprechende Informationen für das 4. Rahmenprogramm.

Abbildung 5.1: Kooperationsprojekte pro 1000 Forscher (1984-96) und nationale F\&EQuoten

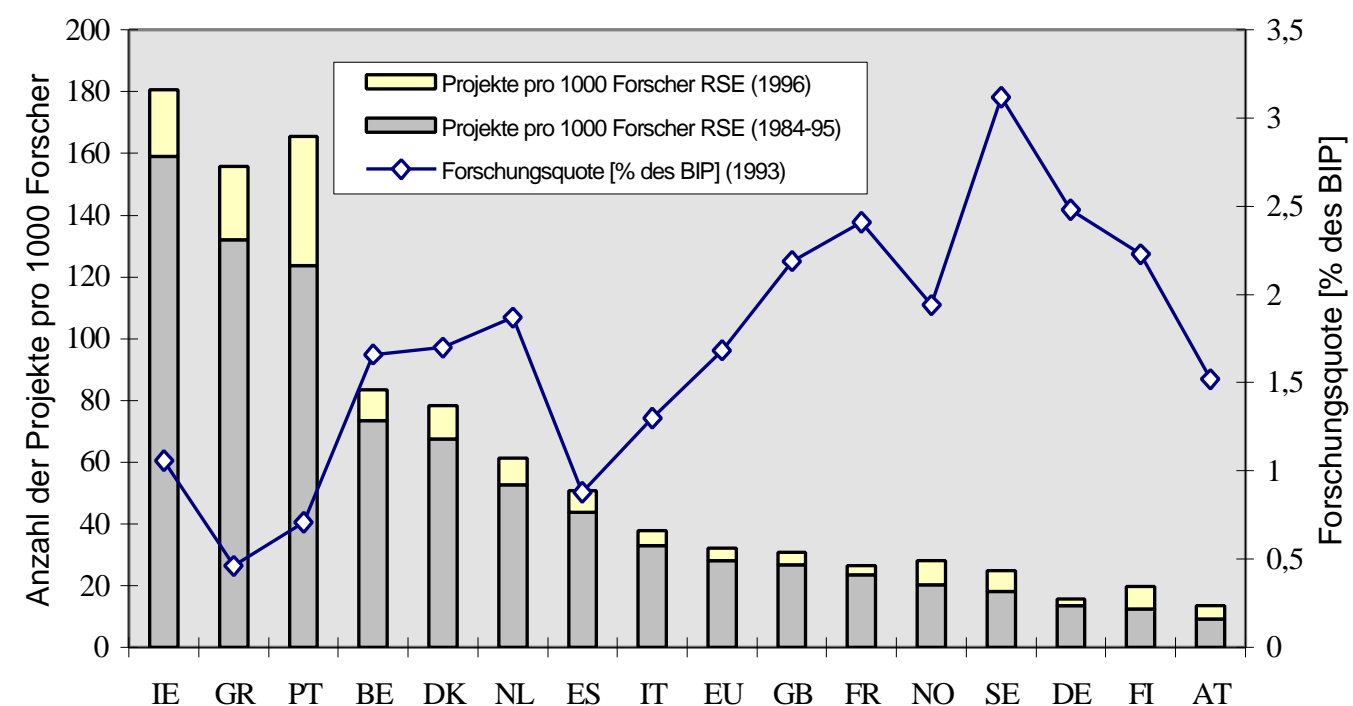

Q: CORDIS, OECD nach Ohler et al. (1997) 
Abbildung 5.2: Aufholprozeß in der Beteiligung an Kooperationsprojekten

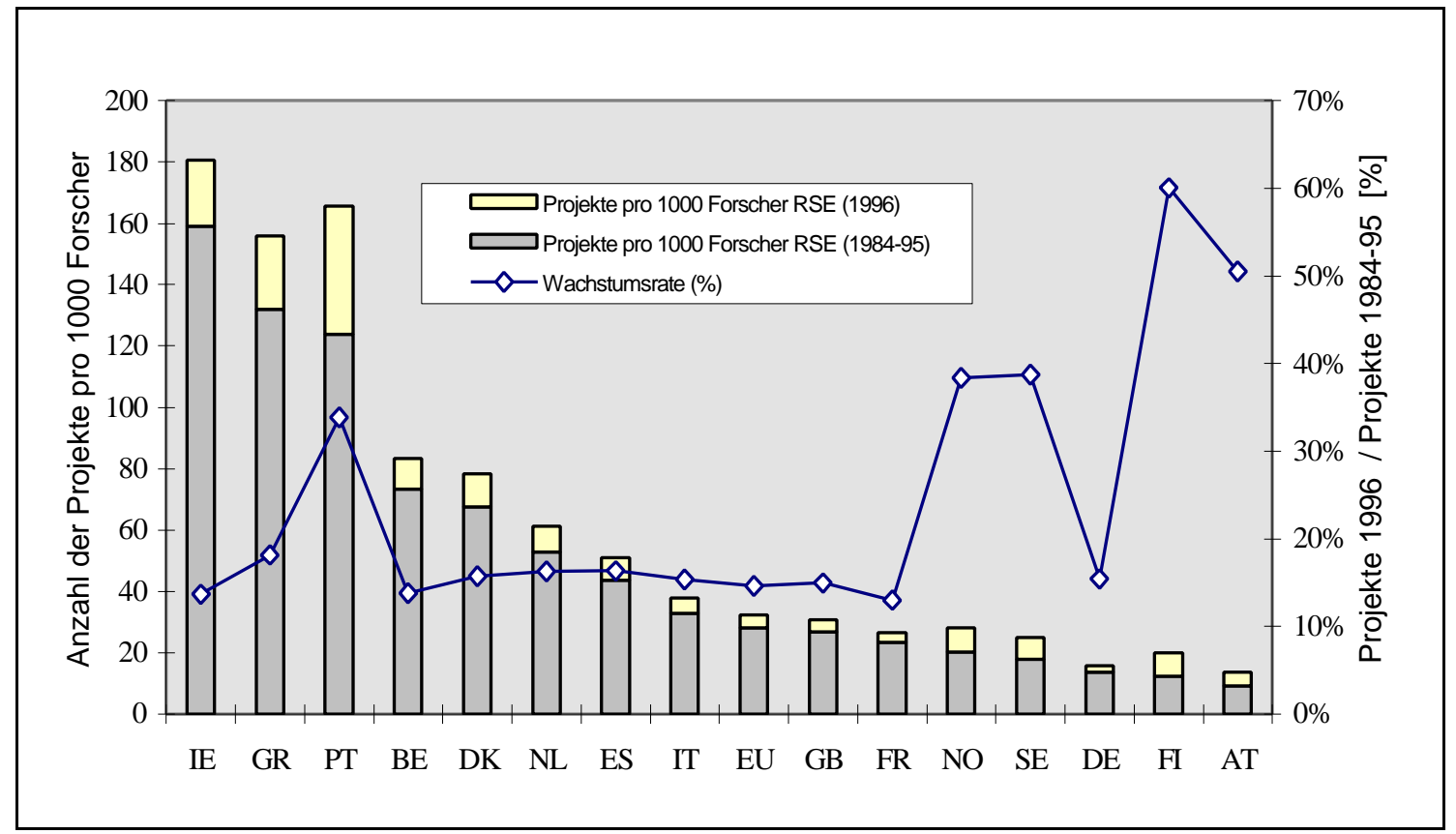

Q: CORDIS, OECD nach Ohler et al. (1997)

Teilnahme nach Organisationstyp: Universitätsinstitute und Forschungseinrichtungen dominieren anfänglich

Die meisten Programme innerhalb der Rahmenprogramme sind für alle Arten von Organisationen offen. Allerdings läßt sich beobachten, daß je nach Programm und je nach Land beachtliche Unterschiede in der Verteilung vorliegen. Die Untersuchung zeigt einige recht aufschlußreiche Charakteristika: Wie erwartet lag zum Zeitpunkt der Durchführung der Evaluierung und kumuliert über den gesamten Beteiligungszeitraum der Anteil von Firmen mit $21 \%$ deutlich unter jenem aus der Forschungsbereich (72\%). Der Anteil der Universitätsinstitute (53\%) war von allen europäischen Ländern am höchsten, während der Anteil der Vertragsforschungseinrichtungen (19\%) zwar im europäischen Durchschnitt lag, angesichts ihrer unterdurchschnittlichen Größe in Österreich aber recht beachtlich war. Der hohe Anteil der Universitätsinstitute und Vertragsforschungseinrichtungen kann im Sinne eines hohen Potentials für einen selbststeuernden und autonomen Prozeß innerhalb des österreichischen Innovationssystems interpretiert werden. Daraus folgt, daß Universitätsinstitute und Vertragsforschungseinrichtungen als Katalysator für die Beteiligung von Unternehmen eine nicht unbedeutende Rolle bei der Intensivierung der österreichischen Beteiligung spielen könnten. Öffentliche Organisationen waren mit beachtlichen $7 \%$ beteiligt. Ein grundsätzlich verschiedenes Bild ergeben die aktuellen Daten über die österreichische Beteiligung am 4. Rahmenprogramm (siehe Box 3), denen zufolge die Unternehmen jetzt die führende Position einnehmen. 
Beteiligung nach Programmgenerationen: Durchbruch im 4. Rahmenprogramm

Der Status Österreichs in den sukzessiven Generationen von Rahmenprogrammen ist von großen Unterschieden gekennzeichnet. Dazu kommt, daß die Rahmenprogramme ihrerseits weitreichende Transformationen erfahren haben. Es ist daher interessant, das Beteiligungsverhalten über die vier Generationen von Rahmenprogrammen hinweg zu studieren. Um diese Analyse durchzuführen, wurde aber nicht vom Ersten bis zum Vierten Rahmenprogramm durchgezählt, sondern es wurden folgende drei Gruppen definiert: (i) Newcomers, die sich im Vierten Rahmenprogramm erstmals beteiligt haben, (ii) Drop-outs, die an einem früheren Rahmenprogramm bereits teilgenommen haben, nicht aber im Vierten Rahmenprogramm und (iii) Old Boys, die im Vierten Rahmenprogramm und in mindestens einem früheren Rahmenprogramm beteiligt waren. Die Ergebnisse sind sehr deutlich und in Hinblick auf die Entwicklung ausgesprochen positiv. Das Erste und Zweite Rahmenprogramm sind zahlenmäßig ohne Bedeutung, das Dritte Rahmenprogramm ist der ernsthafte Einstieg in die Rahmenprogramme, das Vierte Rahmenprogramm kann definitiv als Durchbruch angesehen werden: 61\% aller Teilnehmer sind Newcomers. Die Gruppe der Old Boys ist mit $28 \%$ zweieinhalb mal so groß wie die der Drop-outs (11\%). Diese Gruppe der Old Boys, in der sich vor allem Universitätsinstitute und Vertragsforschungseinrichtungen versammeln, können als die "Europäer" des österreichischen Innovations- und Forschungssystems bezeichnet werden. 
Box 3: Österreichische Beteiligung am 4. Rahmenprogramm für Forschung, technologische Entwicklung und Demonstration der EU - Aktuelle Zahlen und Fakten

1. Die österreichische Beteiligung an finanzierten Projekten: 1.150 Projekte mit knapp 1.460 österreichischen Organisationen haben inzwischen die Finanzierungszusage durch die Europäische Kommission erhalten. Die Beteiligung umfaßt 39\% Unternehmen, $36 \%$ Universitäten, 18\% außeruniversitäre Forschung, 10\% Sonstige. Rund 380 österreichische Unternehmen sind beteiligt, 75\% davon beteiligten sich bisher an einem, $25 \%$ an mehr als einem Projekt. Bemerkenswert ist, daß Firmen an EU-Projekten teilnehmen, die bisher keine österreichische Forschungsförderung in Anspruch genommen haben. $21 \%$ der Beteiligungen von Unternehmen erfolgt im Rahmen der speziell auf die Bedürfnisse von KMU mit geringem F\&E-Potential ausgerichteten Aktion CRAFT.

2. Erfolgsraten und Schwerpunkte: Die durchschnittliche Erfolgsrate österreichischer Projektanträge liegt bei $30 \%$, über diesem Durchschnittswert liegen die Unternehmen (33\%) und die Gruppe der Sonstigen (34\%). Die Universitäten liegen bei 27\% ebenso wie die außeruniversitäre Forschung (27\%). Die Erfolgsrate in der CRAFT-Aktion für KMUs liegt über $50 \%$. Unternehmen halten die höchsten Anteile an den gesamten österreichischen Teilnahmen in den Programmen THERMIE Typ A (EnergieDemonstrationsprojekte, 80\%), ESPRIT (Informationstechnologie 62\%), sowie BriteEuRam (Industrielle und Werkstofftechnologien, 65\%). Die Programme mit dem höchsten Anteil an universitärer Beteiligung sind MAST (Meereswissenschaften, 80\%), BIOMED (74\%), Biotech (67\%), Umwelt (52\%). Die außeruniversitäre Forschung hält den höchsten Anteil im Programm TSER (Sozio-ökonomische Schwerpunktforschung, 50\%) und liegt im Programm FAIR (Landwirtschaft, 37\%) an zweiter Stelle; Die Gruppe der Sonstigen liegt mit $76 \%$ aller österreichischen Beteiligungen im Programm THERMIE Typ $\mathrm{B}$ (Begleitmaßnahmen für Energie-Demonstrationsvorhaben) eindeutig an erster Stelle.

3. Bisher zugesprochene EU-Mittel: Österreichischen Organisationen wurden im 4. Rahmenprogramm von der Europäischen Kommission bisher rund 2,23 Mrd. S zugesprochen - dies entspricht einem Rückfluß von knapp $70 \%$. Auf die Organisationstypen verteilen sich diese Mittel wie folgt: 42\% Unternehmen, 36\% Universitäten, 15\% außeruniversitäre Forschung, 7\% Sonstige.

4. Das Kooperationsverhalten österreichischer Teilnehmer: An den Projekten mit österreichischer Beteiligung sind im Durchschnitt 8 Partner beteiligt - rund 1,5 Österreicher und 6,5 Ausländer. Die Organisationen verteilen sich wie folgt auf die Partnerländer: 20\% DE, 13\% UK, 11\% FR, 10\% IT, 7\% NL, 6\% ES, 5\% BE, 5\% SE, 4\% GR, 3\% SF, 3\% DK, 2\% PT, 2\% IR und Sonstige. 185 finanzierte Projekte werden von österreichischen Koordinatoren geleitet. Der höchste Anteil an österreichischen Koordinatoren findet sich in den Programmen Verkehr (28\%), THERMIE (EnergieDemonstrationsprojekte, mit 26\%) und ESPRIT (Informationstechnologie, mit 23\%), bzw. innerhalb der 2. Aktionslinie im Programm INCO-DC (Kooperation mit Entwicklungsländern, mit 43\%). Nicht-österreichische Koordinatoren von Projekten mit österreichischer Beteiligung verteilen sich wie folgt: 29\% DE, 19\% UK, 11\% FR, 10\% NL, $10 \%$ IT, 4\% BE, 4\% SE, 3\% ES, 3\% DK, 2\% GR, 2\% SF, 2\% IR, 0,5\% PT. 
Quelle: M. Horvat, J. Säckl, BIT-Datenbank INNOman, Stand Jänner 1998.

Kooperative FTE und Strategie: Ziele und strategische Orientierungen von Teilnehmern und Nichtteilnehmern

Eine zentrale Frage in der Bewertung der Effekte kooperativer FTE betrifft die Selektivi tät der Programme hinsichtlich der Ziele und strategischen Orientierung teilnehmender gegenüber nicht-teilnehmenden Organisationen. Das herausragendste Ergebnis ist hier, daß teilnehmende Firmen teilnehmenden Nicht-Firmen (Universi tätsinstitute, Forschungsorganisationen, öffentliche Einrichtungen) ähnlicher sind als nichtteilnehmenden Firmen, d. h. Teilnehmer weisen untereinander einen höheren Grad an Gemeinsamkeiten in ihren strategischen Orientierungen (und Überzeugungen) auf als nichtteilnehmende Organisationen der gleichen Art.

Teilnehmende Organisationen aller Art weisen ferner im Vergleich zu nicht-teilneh menden einen höheren Grad an Integriertheit in ihrer strategischen Orientierung auf: Sie sind auf ein breiteres Spektrum an Zielen gerichtet und verfolgen diese mit größerer Ambitioniertheit. Teilnehmer aller Art messen eine höhere Bedeutung jenen Aktivitäten zu, wo systemisches und implizites, nicht-kodifiziertes Wissen (tacit knowledge) sowie die Integration unterschiedlicher Wissensbestände und unterschiedlicher Kulturen eine bedeutende Rolle spielen. Teilnehmer leben im Vergleich zu Nichtteilnehmern in einer "systemischen Welt" bezug auf die Ziele und strategischen Orientierungen, die sie mit ihrer FTE verfolgen: Integration von angewandter und Grundlagenforschung, Entwicklung neuer und Verbesserung bestehender Methoden und Prozesse eher als Produktentwicklung, Transfer von Technologien und Wissen, Systemintegration etc. Insgesamt läßt sich bei den Teilnehmern in hohem Ausmaß das Vorliegen des Modells der Transfer Sciences in der Wissensproduktion verifizieren.

\section{Kooperationskultur: Voraussetzung, nicht Folge}

Die Rahmenprogramme sind von ihrer Konstruktion her auf kooperative FTE ausgerichtet. Eine interessante Frage ist, ob die Rahmenprogramme ceteris paribus bevorzugt von solchen Organisation angenommen werden, die generell eine höhere Kooperationskultur aufweisen und Kooperation als Teil ihrer Kultur verstehen oder ob die Rahmenprogramme dazu beitragen, Kooperationen zu stimulieren. Die Untersuchungsergebnisse begünstigen sehr deutlich die erstere Vermutung: Rahmenprogramme ziehen vor allem solche Organisationen an, die mit Kooperation vertraut sind und auch außerhalb der Rahmenprogramme regelmäßig kooperieren. Diese Beobachtung hat weitreichende Konsequenzen: (i) Rahmenprogramme können grundsätzlich nicht als Katalysator für Kooperationen herangezogen werden, wo nicht vorher schon Kooperationserfahrung und -neigung existieren. Kooperationserfahrung ist daher die Bedingung für Beteiligung in Rahmenprogrammen und nicht das Ergebnis. (ii) Die Kluft zwischen kooperierenden und nicht-kooperierenden Organisationen kann daher durch die Rahmenprogramme nicht 
überwunden werden, sondern nur durch andere Maßnahmen bzw. überhaupt nicht. (iii) Rahmenprogramme führen möglicherweise sogar zu einer Vergrößerung der Kluft. Um dies zu vermeiden, sind nationale Anstrengungen bzw. spezifische Maßnahmen der Kommission erforderlich.

\section{Erstkontakt mit EU Partnern}

Ein herausragendes Ziel der Rahmenprogramme ist die Stimulierung und Förderung von FTE Netzwerken zwischen verschiedenen Ländern, Branchen, Organisationstypen und Wissensträgern. Eine in diesem Zusammenhang vordringliche Frage ist, ob Rahmenprogramme in der Lage sind, wesentlich zur Bildung neuer Netzwerke beizutragen und Partner zusammenzuführen, die vorher nicht kooperiert haben. Die Unter suchung liefert hierzu sehr deutliche Ergebnisse: Es ist die überragende Bedeutung der Mitgliedschaft in bereits existierenden Netzwerken. Die Rahmenprogramme tragen entweder zur Festigung bestehender Netzwerke bei oder sie stimulieren neue Konfigurationen innerhalb der schon bestehenden. Dieses Ergebnis sollte jedoch nicht zu Pessimismus verleiten: Die Mitgliedschaft in zwei verschiedenen Netzwerken schafft das Potential zur Verbin dung beider und daher zur Schaffung eines neuen Netzwerks. Aus diesem Grund impliziert die Wichtigkeit der Mitgliedschaft in bestehenden Netzwerken nicht, daß durch die Rahmenprogramme keine neuen Netzwerke geschaffen werden. Es liegt eher eine Bestätigung der altbekannten Tatsache aus der Innovationstheorie vor, wonach Innovation gewöhn lich aus der neuen Kombination bestehender Teile (von Wissen) entsteht.

Linking up to Europe: Erwartungen, Motive, Ziele und Bedeutung von EU-Projekten

Eine häufig vorgetragene Kritik an den Rahmenprogrammen ist die vorgeblich schwache Übereinstimmung der Ziele und Erwartungen der teilnehmenden Firmen und Instituten und jenen der Kommission. Die letzteren sind gut bekannt - es sind dies (i) thematische Ziele, dargelegt in den spezifischen Programmen und (ii) prozeßbezogene Ziele wie Kosten- und Risikoteilung, Kooperation oder Technologietransfer. Die Ziele der individuellen Teilnehmer mögen in Übereinstimmung mit jenen der Kommission sein, aber durchaus auch nicht. Empirische Untersuchung ist daher erforderlich, die Ergebnisse sind die folgenden:

Mehr als die Hälfte der Ziele werden als mäßig bis sehr wichtig eingestuft. Sehr wichtig dagegen sind nur einige wenige, und zwar solche, die überwiegend (i) auf Zugang (u. a. zu Finanzierung, Expertise, Forschungseinrichtungen), (ii) auf Kompetenzaufbau und (iii) auf Netzwerkbildung ausgerichtet sind. All diese Ziele sind expansionistisch, reduktionistische Motive wie Kosten- und Risikoteilung sind insgesamt von geringer Bedeutung. Dennoch sind im einzelnen Unterschiede zwischen verschiedenen Organisationstypen festzustellen:

Mehr als 50\% aller Firmen betrachten Kostenteilung, Zugang zu zusätzlicher Finanzierung, Aufbau von Kooperationen mit europäischen Firmen, Imageaufwertung, Entwicklung/Verbesserung von Prozessen, Prototypenbau, Exploration alternativer technologischer Pfade und Risikoteilung als sehr wichtig. Das Ausmaß der Enttäuschung 
über nicht erreichte Ziele ist ausgesprochen gering. Andererseits gibt es einen "Überschuß" an Zielerreichung. Rund ein Drittel der Firmen berichten einen hohen Grad an Erreichung bei solchen Zielen, die ihrerseits von geringer Relevanz sind. Dies kann als nichtintendierter Zusatznutzen betrachtet werden, der ein Potential darstellt, welches genützt werden kann und soll.

Nicht-Firmen weisen eine in manchen Punkten von Firmen verschiedene Ziel-/Relevanzstruktur auf: Nicht-Firmen sind in hohem Maße auf die Erreichung von Zugangsmöglichkeiten und Kompetenzaufbau gerichtet. Gleichzeitig sind sie darin sehr erfolgreich und weisen eine dementsprechend niedrige Frustrationsrate auf. Zusatznutzen ergibt sich vor allem für den Ausbau der Fertigkeiten des involvierten Personals, im Vertrautwerden mit Tools und Techniken bzw. mit Standards sowie im Eingehen neuer Partnerschaften. Auch hier sind zusätzliche Aktivitäten erforderlich, um diese entsprechend zu nützen.

Bei der Einführung der Unterscheidung zwischen Old Boys und Newcomers bestand ein wesentliches Motiv darin, zu zeigen, daß Old Boys einen höheren Grad an Erreichung bei wichtigen Zielen aufweisen. Die zugrundeliegende Hypothese war, daß Old Boys eine höhere Konzentration auf wenige Ziele aufweisen, weil sie aus Erfahrung wissen, was erreichbar ist und was nicht. Der Vergleich indes zeigt überraschende Ergebnisse: Old Boys und Newcomers weisen weitgehend dieselbe Verteilung von Zielerreichung und Zielrelevanz auf, es ist daher kein signifikanter Vorteil durch Lernen erkennbar - mit einigen Ausnahmen: Old Boys sind zurückhaltender hinsichtlich marktorientierter Ziele und daher stärker motiviert etwa von Kompetenzaufbau, was als Hinweis darauf zu werten ist, daß erfahrenere Teilnehmer den Wert der intangiblen Erträge kooperativer FTE mehr zu schätzen wissen.

Forschen in regulären und EU-Projekten: Gemeinsamkeiten überwiegen

Eine sehr allgemeine Forderung an die öffentliche Finanzierung von Forschung ist die Erreichung eines Mindestmaßes an Additionalität, etwa in Form der Durchführung risikoreicherer, kostenintensiverer, längerfristiger Projekte, Projekte in (peripheren) Gebieten, die sonst nicht durchgeführt würden, Behandlung komplexerer Fragen etc. Die Untersuchung liefert hierzu wertvolle und weit hin überraschende Ergebnisse: Es zeigt sich, daß die Arbeit in EU-Projekten kaum von der Arbeit in regulären Projekten abweicht, ausgenommen davon ist die Erfordernis der Zusammenarbeit. Auf grund dieser geringen Abweichung zwischen EU-Projekten und regulären Projekten, tragen dementsprechend auf den ersten Blick - EU-Projekte wenig zu einer Umorientierung der Forschung insgesamt bei.

Dieses in hohem Maß unerwartete Ergebnis kann jedoch durch die Mechanismen und Prozeduren der Projektevaluation und -auswahl gut nachvollzogen werden: Die Qualität der Anträge hängt wesentlich von der Ambitioniertheit, den Fähigkeiten und der Erfahrung der involvierten Personen und Organisationen ab. Um im harten Wettbewerb der Anträge bestehen zu können, müssen die Antragsteller ihr bestes Personal, ihre besten Fähigkeiten, 
Ideen usw. mobilisieren, was nichts anderes heißt als sich auf die Kernkompetenzen zu konzentrieren. Daraus ergibt sich unmittelbar, daß die Art und Weise der Projektbewertung und -auswahl jene Organisationen begünstigt, die genau jene Forschung durchzuführen beabsichtigen, mit der sie am meisten vertraut sind, also Forschung innerhalb ihrer Kernaktivitäten und -kompetenzen.

Die strategische Bedeutung von EU-Projekten

Hat die hohe Ähnlichkeit zwischen regulären und EU-Projekten zur Folge, daß diese auch einen starken strategischen Zusammenhalt aufweisen? Tatsächlich kann ein derartiger Zusammenhang nachgewiesen werden: Bei einem Drittel jener Organisationseinheiten, die an mehr als einem Projekt teilgenommen haben, sind die Projekte thematisch/strategisch eng verbunden, was als Ausdruck einer sehr expliziten und strategischen Herangehensweise an die Rahmenprogramme gewertet werden kann. Die Hälfte der Teilnehmer mit mehreren Projekten sehen zumindest einen losen Zusammenhang zwischen den einzelnen EU-Projekten. Bemerkenswert ist auch die Tatsache, daß drei Viertel aller Teilnehmer an mehr als einem Projekt teilgenommen haben. Dies be deutet, daß der Großteil der österreichischen Teilnehmer EU-Projekte nicht als beiläufige Aktivität von geringer Relevanz ansehen, sondern innen eine hohe strategische Bedeutung beimessen. Was den Zusammenhang zwischen regulären und EU-Projekten betrifft, so ergibt sich ein ähnliches Bild: $57 \%$ berichten einen starken, $41 \%$ immerhin einen losen Zusammenhang zwischen beiden Projekttypen. Alles in allem kann von einem guten strategischen "Fit" zwischen beiden Projektklassen gesprochen werden, was seinerseits einen erfolgreichen Wissenstransfer garantieren sollte.

Wissens- und Technologietransfer und Nutzung

Transfer von Technologie und Wissen erfolgt in unterschiedlichsten Formen. In Bereichen, in denen ein hoher Grad an nicht-kodifiziertem, implizitem Wissen vorherrscht und das lineare Modell des Innovationsprozesses durch das Modell der Transfer Sciences abgelöst wird, ist kooperative FTE überhaupt die Voraussetzung für Transfer und Anwendung. Dementsprechend ist diese Frage von zentraler Bedeutung für die Relevanz der Beteiligung von EU-Projekten. Dies sind die Ergebnisse: Die Hälfte der Teilnehmer geben einen Transfer innerhalb ihrer Organisationseinheit an. Ein Drittel der Firmen und die Hälfte der Nicht-Firmen haben Ergebnisse nach außen transferiert, der Transfer nach innen liegt in derselben Größenordnung. Angesichts der Tatsache, daß die meisten Teilnehmer Newcomer sind und das Vierte Rahmenprogramm erst kurz angelaufen ist, ein recht erfreuliches Ergebnis. 
Teilnehmer haben also eine gute Balance zwischen intra- und inter-organisatorischen Netzwerken. Der wichtigste Ort der Nutzung der Ergebnisse ist aber in jedem Fall die eigene Einheit sowie die der Partner. Diese Symmetrie ist übrigens ein Hinweis auf das Bestehen von Know-how-sharing und die Abwesenheit von Freeridership, d. h. es gelingt in EU-Projekten kaum, Ergebnisse zu nutzen ohne selbst welche einzubringen.

\section{Erfolgs- und Mißerfolgsfaktoren}

Erfolg ist nicht durch Abwesenheit von Mißerfolg garantiert, Erfolgs- und Mißerfolgsfaktoren können durchaus nebeneinander bestehen. Empirische Untersuchung kann hier helfen, die Verhältnisse dieser beiden Faktorenbündel aufzudecken. Dies sind die Befunde: Kompetenz der Partner (einschließlich der eigenen) ist der wichtigste Erfolgsfaktor, der zusammen mit ambitionierten technischen Zielen, den "Brennstoff" für Projekterfolg ausmacht. Eine zweite wichtige Gruppe von Erfolgsfaktoren bildet die Managementkompetenz: Projektmanagement durch den Koordinator, eigenes Projektmanagement, Koordination zwischen den Partnern und die Struktur des Konsortiums rangieren ebenfalls hoch. Hemmende Faktoren, soweit es sie überhaupt gibt, resultieren vorwiegend aus hohen Transaktions- und Kommunikationskosten sowie von beabsichtigten und unbeabsichtigten Veränderungen der Projektstruktur (siehe Abbildung 5.3).

Abbildung 5.3: Erfolgs- und Mißerfolgsfaktoren nach Einsteigern, Old Boys und Aussteigern

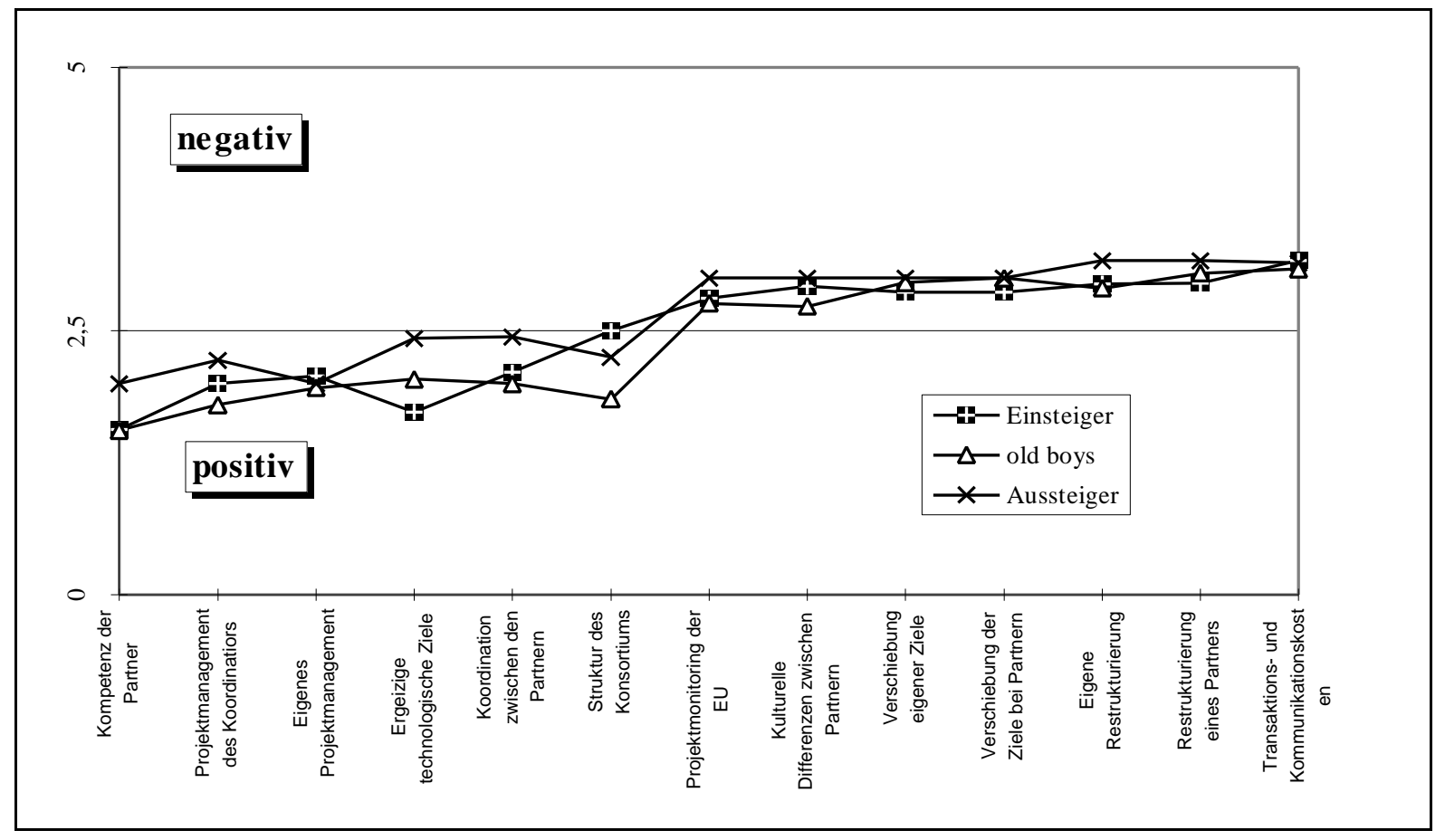

Q: Ohler et al. (1997) 
Fortführung von abgelehnten Projekten

Die Erfolgsrate von eingereichten Projekten liegt zwischen $1: 3$ und 1:12. Dem entsprechend ist die Zahl der abgelehnten Projekte und die mit deren Vorbereitung verbundenen Kosten hoch. Die Frage stellt sich, ob die bereits investierten Ressourcen als "Sunk Costs" betrachtet oder in anderen Projekten verwendet werden, mit einer anderen Finanzierung, in einem anderen Maßstab oder mit anderen Partnern. Dies sind die Beobachtungen: Drei Viertel der Antragsteller führen abgelehnte gelegentlich weiter, ein Drittel oft bis immer. Weiterführung der Kooperation mit den gewählten Partnern ist eher die Regel als die Ausnahme, allerdings nicht mit allen Partnern und mit reduzierten Zielsetzungen und durchaus mit Förderung aus anderen Quellen. Dieses Ergebnis befindet sich in Übereinstimmung mit der Beobachtung, daß die Teilnahme an Rahmenprogrammen eher existierende Netzwerke stärkt denn neue schafft bzw. mit der Tatsache, daß die gemeinschaftlich durchgeführten Aktivitäten nicht unähnlich den regulär durchgeführten Forschungen sind und daher im Kernbereich liegen.

Die Tatsache, daß das durchschnittliche Projektkonsortium die einmal geplante und detailliert vorbereitete Arbeit auch ohne EU Finanzierung fortführt, kann als Evidenz für einen hohen Grad an Additionalität gewertet werden. Sie bestätigt die Trigger-Hypothese, wonach die bloße Existenz der Rahmenprogramme (bei gegebenem Finanzierungsvolumen) ein mehrfaches Volumen an FTE-Tätigkeit auslöst. Diese Hypothese setzt allerdings voraus, daß ohne die Existenz der Rahmenprogramme die entsprechenden Aktivitäten überhaupt nicht in Angriff genommen worden wären, was im folgenden auch gezeigt wird.

\section{Einfluß der EU Förderung}

Öffentliche Förderung von FTE sollte Aktivitäten auslösen, die anderenfalls nicht durchgeführt würden. Was auf theoretischer Ebene leicht gesagt ist, kann empirisch oft nur schwer nachgewiesen werden. Hier einige Beobachtungen über den Einfluß der EU Förderung: Grundsätzlich gibt es einen hohen Grad an Additionalität. Vertragsforschungseinrichtungen als extremster Fall antworteten zu 84\%, daß sie ohne die Aussicht auf EU Förderung die Projekte nicht durchgeführt hätten. Unternehmen und Universitätsinstitute folgen generell diesem Verhalten, wenngleich nicht in dersel ben Höhe.

Additionalitäten: Mehrfach-Spill-overs von EU Programmen

Diese Beobachtungen fügen zwei komplementäre Formen von Additionalität zusammen: Rahmenprogramme stimulieren und finanzieren FTE-Tätigkeiten, die ansonsten nicht durchgeführt würden. Sind die Projektvorbereitungen einmal getroffen, so führen diese, auch wenn sie nicht von der EU finanziert werden, zu weiterführenden Aktivitäten. Damit ergeben sich drei bzw. vier Finanzierungsströme, die direkt oder indirekt durch die EU Programme beeinflußt werden: (i) Die Finanzierung durch die EU, (ii) der eigenfinanzierte 
Anteil durch die Teilnehmer, (iii) die Finanzierung jener Aktivitäten, die in Erwartung einer EU Finanzierung vorbereitet wurden, aber nunmehr anderweitig finanziert werden und schließlich (iv) jene Aktivitäten, die durch die EU-Projekte ausgelöst werden und dann allein, bilateral oder in neuen Konsortien weitergeführt werden, wobei letzteres durch das Old Boys Phänomen bestärkt wird.

\section{Substitution oder Komplementarität zwischen nationalen und EU Programmen}

Subsidiarität als konstitutives Element der Europäischen Union, sollte tunlichst auch im Bereich der Forschungs- und Technologiepolitik gelten. Daß dies der Fall ist, läßt sich rasch zeigen: Von den 49 eingelangten Antworten teilen nur 9 die Auffassung, daß EU Programme nationale Programme substituieren. Dies kann als eine spezifische Form von Additionalität im Bereich der Forschungs- und Technologiepolitik betrachtet werden und sollte die österreichischen Bundes- und Landesstellen davon abhalten, mit Verweis auf die Existenz der EU Programme die nationalen und regionalen Programme zu kürzen.

\section{Barrieren in der Teilnahme an EU Programmen}

Nichtteilnehmer wurden nach den maßgeblichen Hindernissen für eine Teilnahme an EU Programmen gefragt. Die Ergebnisse sind in mancher Hinsicht überraschend: Als stärkste Hindernisse werden die komplizierte Antrags- bzw. Abwicklungsprozeduren bezeichnet (61 bzw. 59\%). Diese Resultate signalisieren einen hohen Grad an Bereitschaft, da die Hauptbarrieren im Prinzip überwindbar und daher diese Gruppe als potentielle Teilnehmer zu betrachten sind. Es handelt sich insofern um weiche Barrieren. Harte Barrieren dagegen tun sich für solche Organisationen auf, die eine schwache Übereinstimmung mit den eigenen Technologiestrategien sehen (40\%), denen die Erträge aus der Kooperation zu gering sind $(34 \%)$ und die wegen der hohen Ablehnungsquote nicht teilnehmen wollen $(30 \%)$ - für alle diese Organisationen sind Rahmenprogramme grundsätzlich von geringer Relevanz. Alle anderen denkbaren Hinderungsgründe sind weitgehend vernachlässigbar, weil sie unter ein Viertel der Nennungen fallen. Alles in allem sind Nichtteilnehmer einer künftigen Teilnahme zugeneigt, woraus sich eine Abschätzung von zusätzlichen $300-400$ Organisationen als Potential für künftige Teilnahme ergibt.

Künftige Teilnahme an EU-Projekten von Nichtteilnehmern: Hohe Bereitschaft

Nichtteilnehmer wurden nach ihrer Absicht, künftig an Rahmenprogrammen teilzunehmen, gefragt. Die Ergebnisse sind sehr deutlich: 84\% aller Nichtteilnehmer haben eine Teilnahme vor, vor allem bei Vertragsfor schungseinrichtungen, aber auch bei Universitätsinstituten und kleineren wie größeren Unternehmen. Das Potential aufgrund dieser Schätzung liegt bei rund 400 Organisationen. 
Nationale Forschungs- und Technologieprogramme und EU Programme: Ihr Antragsverhalten im Vergleich

Ein Vergleich des Antragsverhaltens bei maßgeblichen österreichischen Forschungs- und Technologieförderprogrammen des FFF und ITF mit jenen der EU liefert weitere Informationen über das Potential für künftige Teilnahme. Das Ergebnis ist eine Zahl von 150 - 200 Firmen, die als Potential für EU Programmbeteiligungen anzusehen sind. Nicht inkludiert in diese Schätzung sind jedoch solche Firmen, die im Rahmen von sogenannten special actions oder accompanying measures angesprochen werden können.

Quantifizierung des Potentials für künftige Beteiligungen an Rahmenprogrammen

Die verschiedenen Methoden zur Abschätzung des künftigen Potentials für EU Programmteilnahme liefern recht robuste Ergebnisse. Sie liegen in der selben Größenordnung und konvergieren hin zu einer Zahl von 200 Firmen und 300 Instituten (Universitätsinstitute und Vertragsforschungseinrichtungen). In diese Schätzung sind aber nicht die erwähnten Zielgruppen von special actions oder accompanying measures berücksichtigt. Mag sein, daß das Potential größer ist. Nichtsdestoweniger ist die Mobilisierung dieser 500 Organisationen, von denen die Mehrzahl mehrere Projekte durchführt, eine durchaus beachtliche Herausforderung für die involvierten Ministerien, Transfer- und Koordinationseinrichtungen.

EU Programme: Die Transformation der Forschungs- und Technologiepolitik

"Brüssel" - ein anderes Synonym für Bürokratie und die Europäische Kommission als ihre operative Basis, die Rahmenprogramme als Inkarnation von top-down-Planung der Forschungs- und Technologiepolitik. Halten Ansichten wie diese der empirischen Untersuchung stand? Interviews mit österreichischen Entscheidungsträgern auf unterschiedlichen Ebenen der nationalen und EU-Politik zeigen in der Tat ein deutlich differenzierteres Bild, ein Bild wohl auch, das mehr Licht auf die na tionale Politik wirft als auf die der Kommission: Es gibt kein einheitliches 'Brüssel'. Vielmehr gibt es eine große Zahl von Verhaltensweisen und daher einen sehr verschieden gearteten Bedarf an Lernen und Handeln. Während die Delegierten in der kurzen Zeit ihrer Vollteilnahme diese Prozesse mehr oder weniger absolviert haben und nunmehr auch auf der informellen und formalen Verhandlungsebene Vollmitglieder geworden sind, ist dieser Lernprozeß in bezug auf nationale Akteure und deren Verhältnis zu den EU-Strukturen und -Prozessen bestenfalls auf halbem Wege. Gleichwohl gibt es ein gemeinsames Verständnis darüber, daß dieses Auseinanderklaffen zwischen nationaler und EU-Politik ein Übergangsphänomen darstellt, welches in den nächsten Jahren verschwinden wird. Diese Frist fällt im übrigen mit der Verabschiedung des Fünften Rahmenpro gramms zusammen, was die Verantwortlichen, vor allem auf nationaler Ebene, nicht von der Auf gabe entläßt, die verschiedenen (Sektor-)Politiken mit jenen der EU stärker und expliziter abzu stimmen. 
EU und nationale Forschungs- und Technologiepolitik: Lern- und Transferprozesse

Die Involvierung in Prozesse der EU fungiert in vielfacher Weise als Nährboden für nationale Forschungs- und Technologiepolitik. Die Delegierten sind in hohem Maße angetan von den Möglichkeiten, Erfahrungen zu sammeln, nicht nur für ihre eigene Aufgabe etwa in den Programm-Management-Komitees, sondern vor allem auch für die nationale Politik. Die am häufigsten angeführten Aspekte sind: (i) Die Bewältigung komplexer Themen und Programme, (ii) die explizitere Pla nung und Abwicklung von Programmen einschließlich der obligatorischen Evaluierung, (iii) neue Zielgruppen für Politikmaßnahmen (öffentliche Einrichtungen, "immaterielle" Firmen wie Consul tants, Konsortien etc.) und (iv) ein weiterer Blick auf Forschungsthemen und Innovationen (Anerkennen von Lernen, Wissen und Fertigkeiten als wesentliche Dimension von Technologie, höhere Aufmerksamkeit für infrastrukturellen Maßnahmen, Diffusion etc.).

Ein sehr spezifischer Effekt ist in diesem Zusammenhang berichtenswert: Die Beteiligung an den Rahmenprogrammen hat nicht nur für die teilnehmenden Organisationen erhebliche Additionalitätseffekte ausgelöst, sondern auch auf der Ebene der Politik, wo eine deutliche Verschiebung gegenwärtig zu beobachten und noch für die nächsten Jahre zu erwarten ist.

\section{Zusammenfassung: Beteiligung an den Rahmenprogrammen schafft Potentiale}

Die Beteiligung an den Rahmenprogrammen der EU hat für alle Beteiligten einen deutlichen Zuwachs an strategischen Möglichkeiten mit sich gebracht. Für die teilnehmenden Firmen, Universitätsinstitute, Forschungsorganisationen und öffentlichen Einrichtungen ist es vor allem die Ausweitung ihrer Zugangsmöglichkeiten - zu Know-how, zu Netzwerken, zu neuen Formen des Forschens und zum Vertrautwerden mit anderen Kulturen und Stilen. Diese soweit aufgebauten Potentiale tatsächlich zu nutzen, ist jedoch nicht Teil der EUProjekte selbst, sondern bedarf zusätzlicher Aktivitäten, sowohl auf Ebene der teilnehmenden Organisationen als auch im Rahmen der nationalen (einschließlich regionalen) Forschungs- und Technologiepolitik. Auf diese Weise ließe sich ein Maximum an Zusatznutzen aus der Teilnahme an EU-Programmen erzielen. Pointiert formuliert heißt dies: Die effiziente Nachnutzung einer eher geringen Beteiligung ist einer emsigen Beteiligung ohne sorgfältige und systematische Nachnutzung vorzuziehen. Diese Betrachtung relativiert das Schielen auf hohe Rückflußquoten und stellt die Qualität der Zugänge und deren Nutzung in den Vordergrund.

Die Beteiligung an den Rahmenprogrammen hat aber auch Implikationen für die nationale Forschungs- und Technologiepolitik: Hier gibt es klare Hinweise auf eine Transformation der nationalen Politik. Zum einen erzwingt die Involvierung in EU-G remien eine stärkere Abstimmung der nationalen Agenden mit jenen der EU. Zum anderen eröffnet die Tätigkeit in den EU-Gremien vielfältige Möglichkeiten des Lernens und des Erfahrungsaustausches. Diese von der Kommission nicht direkt beabsichtigten und vielfach unbemerkten Lern- und 
Transferprozesse sollten nicht unterschätzt werden. Sie stellen ein unverzichtbares Bindeglied zwischen der Kommission und den Mitgliedsstaaten dar, das kaum durch andere Maßnahmen wie Informationskampagnen, Datenbanken und Koordinationsaktivitäten auf Kommissionsebene ersetzt werden kann. 


\section{Literatur}

Aiginger, K., Peneder, M., Qualität und Defizite des Industriestandorts Österreich, Österreichisches Institut für Wirtschaftsforschung, Wien, 1997.

Blomström, M., Kokko, A., "How Foreign Direct Investment Affects Host Countries", The World Bank, Policy Research Paper, 1997, (1745).

Coe, D. T., Helpman, E., "International R\&D Spillovers", European Economic Review, 1995, 39, S. 859-887.

COM(97)142 fin, Proposal for a European Parliament and Council Decision Concerning the $5^{\text {th }}$ Framework Programme of the European Community for Research, Technological Development and Demonstration Activities (1998-2002), Brussels, 9 April 1997.

Gassler, H., Kopcsa, A., Schiebel, E., Böck, N., Gheybi, P., "Verbesserter Einsatz von Patentstatistiken", Österreichisches Forschungszentrum Seibersdorf, 1996, (OEFZS-A-3613b).

EITO (European Information Technology Observatory), Frankfurt am Main, 1997.

Europäische Kommission, Panorama der EU-Industrie 95/96, Luxemburg, 1996.

Europäisches Patentamt (EPA), Jahresberichte 1987-1995, München.

Gilli, P. V., Kurz, R., Streicher, W., Zuschin, B., Industrielle Kraft-Wärme-Kopplung in Österreich (Cogeneration), Schriftenreihe der Energieforschungsgemeinschaft im Verband der E-Werke Österreichs (VEÖ), Wien, 1995.

Hutschenreriter, G., "Intersektorale und internationale 'F\&E-Spillovers'. Externe Effekte von Forschung und Entwicklung", WIFO-Monatsbericht, 1995, 68(6), S. 419-427.

Hutschenreiter, G., Peneder, M., "Österreichs 'Technologielücke' im Außenhandel", WIFO-Monatsberichte, 1997, 70(2), S. 103-114.

Kageson, P., "Getting the Prices Right", T\&E, 1993, (6), Brussels - Stockholm.

Lassnigg, L., Pollan, W., "Das österreichische Qualifizierungssystem im internationalen Vergleich", WIFOMonatsberichte, 1996, 69(12), S. 763-780.

Legler, H., "Zur Position der Bundesrepublik Deutschland im internationalen Wettbewerb", Forschungsberichte des Niedersächsischen Instituts für Wirtschaftsforschung, 1982, (3).

Martinez Sanchez, A., "Innovation Cycles and Flexible Automation in Manufacturing Industries ", Technovation, 1995, 15(6), S. 351-362.

Molitor, R., Alpentransit - Güterzüge statt Lkw-Kolonnen, Wissenschaft \& Verkehr, VCÖ Verkehrsclub Österreich, 1996.

Mooss, H., Industrieroboter-Jahrbuch 1995. Anwenderstatistik 1994 und Herstellerverzeichnis, Schriftenreihe Technologie des Wirtschaftsförderungsinstitutes (WIFI), Wirtschaftskammer Österreich, 1995.

OECD, Frascati Manual, Paris, 1993.

OECD, Education at a Glance. Indicators, Paris, 1997.

OECD, Communication Outlook, Paris, 1997.

Ohler, F., Jörg, L., Polt, W., Guy, K., Hutschenreiter, G., Husz, M., Sieber, A., Gluske, H., Patsios, S., Evaluation of the Austrian Participation in Community RTD Programmes, Seibersdorf Report OEFZS4792, Wien-Seibersdorf, 1997.

Peneder, M., Außenhandel und Innovation. Technologischer Wandel, internationaler Wettbewerb und die Muster der Exportspezialisierung im österreichischen Außenhandel von 1970 bis 1994, tip, Wien, 1995. 
Schulmeister, St., "Das technologische Profil des österreichischen Außenhandels", WIFO-Monatsberichte, 1990, 63(12), S. 663-675.

WIFI, Flexible Automation, Schriftenreihe Technologie, (203), 1989.

UN - IFR (United Nations Economic Comission for Europe and International Federation of Robotics), World Industrial Robots 1996. Statistics 1983-1996 and Forecasts to 1999, New York - Geneva, 1996.

Utterback, J., Abernathy, W., "A Dynamic Model of Product and Process Innovation", Omega, 1975, 3(6), S. 639-656. 